\title{
Müller glia: Stem cells for generation and regeneration of retinal neurons in teleost fish ${ }^{\text {is }}$
}

\author{
Jenny R. Lenkowski**,1, Pamela A. Raymond ${ }^{*, 1}$ \\ Department of Molecular, Cellular, and Developmental Biology, College of Literature, Science, and the Arts, University of Michigan, Ann Arbor, MI, USA
}

\section{A R T I C L E I N F O}

\section{Article history:}

Available online $\mathrm{xxx}$

\section{Keywords:}

Adult neurogenesis

Retinal regeneration

Müller glia

Radial glia

Retinal stem cells

\begin{abstract}
A B S T R A C T
Adult zebrafish generate new neurons in the brain and retina throughout life. Growth-related neurogenesis allows a vigorous regenerative response to damage, and fish can regenerate retinal neurons, including photoreceptors, and restore functional vision following photic, chemical, or mechanical destruction of the retina. Müller glial cells in fish function as radial-glial-like neural stem cells. During adult growth, Müller glial nuclei undergo sporadic, asymmetric, self-renewing mitotic divisions in the inner nuclear layer to generate a rod progenitor that migrates along the radial fiber of the Müller glia into the outer nuclear layer, proliferates, and differentiates exclusively into rod photoreceptors. When retinal neurons are destroyed, Müller glia in the immediate vicinity of the damage partially and transiently dedifferentiate, re-express retinal progenitor and stem cell markers, re-enter the cell cycle, undergo interkinetic nuclear migration (characteristic of neuroepithelial cells), and divide once in an asymmetric, self-renewing division to generate a retinal progenitor. This daughter cell proliferates rapidly to form a compact neurogenic cluster surrounding the Müller glia; these multipotent retinal progenitors then migrate along the radial fiber to the appropriate lamina to replace missing retinal neurons. Some aspects of the injury-response in fish Müller glia resemble gliosis as observed in mammals, and mammalian Müller glia exhibit some neurogenic properties, indicative of a latent ability to regenerate retinal neurons. Understanding the specific properties of fish Müller glia that facilitate their robust capacity to generate retinal neurons will inform and inspire new clinical approaches for treating blindness and visual loss with regenerative medicine.
\end{abstract}

(c) 2014 The Authors. Published by Elsevier Ltd. All rights reserved.

Abbreviations: Acsl1a, achaete-scute like 1a; Alcama, activated leukocyte cell adhesion molecule a; Apobec2a/2b, apolipoprotein B mRNA editing enzyme, catalytic

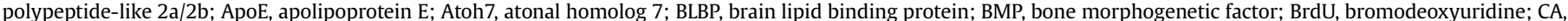

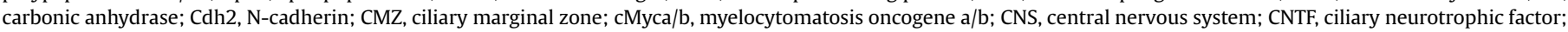

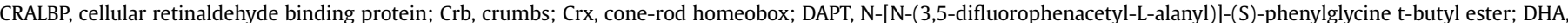

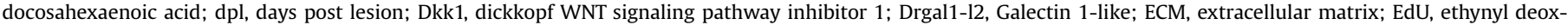

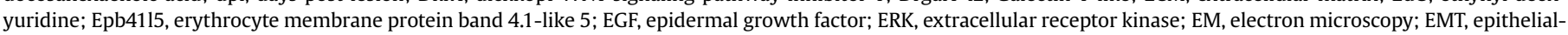

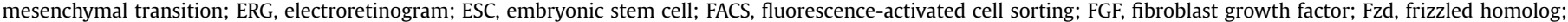

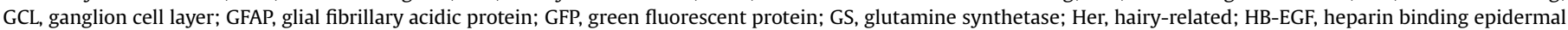

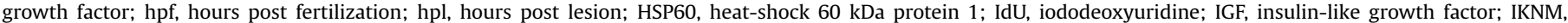

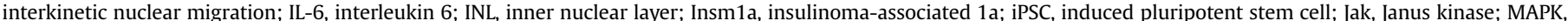

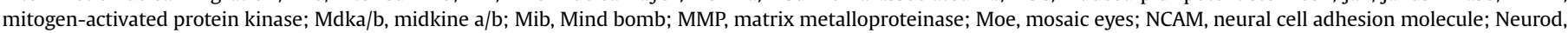

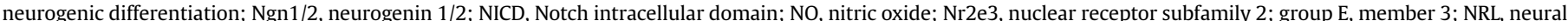

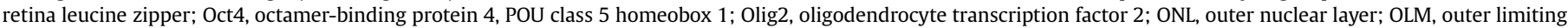

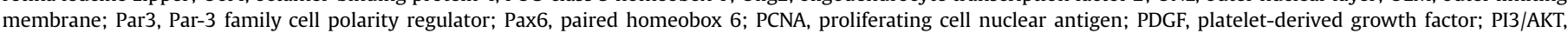

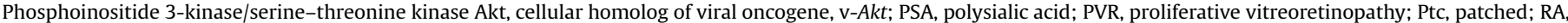

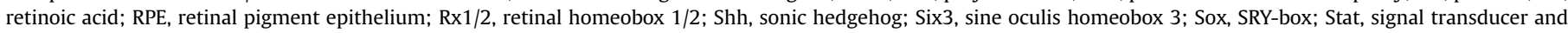

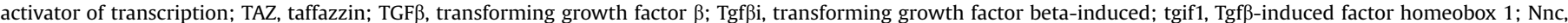

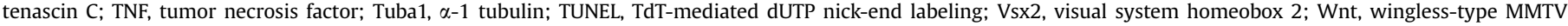
integration site family; YAP, yes-associated protein.

This is an open-access article distributed under the terms of the Creative Commons Attribution-NonCommercial-No Derivative Works License, which permits noncommercial use, distribution, and reproduction in any medium, provided the original author and source are credited.

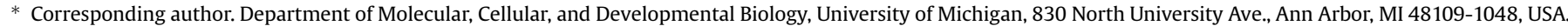
Tel.: +17346470811.

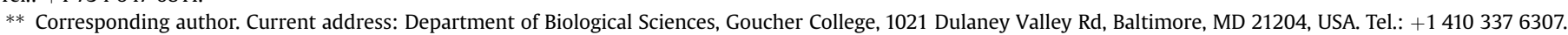
E-mail addresses: jenny.lenkowski@goucher.edu (J.R. Lenkowski), praymond@umich.edu (P.A. Raymond).

1 Percentage of work contributed by each author in the production of the manuscript is as follows: Jenny R. Lenkowski: 50\%; Pamela A. Raymond: 50\%.

1350-9462/\$ - see front matter (c) 2014 The Authors. Published by Elsevier Ltd. All rights reserved.

http://dx.doi.org/10.1016/j.preteyeres.2013.12.007 


\section{Contents}

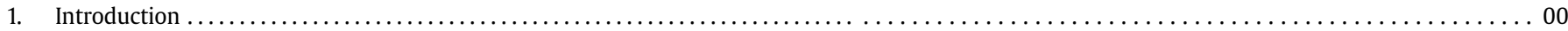

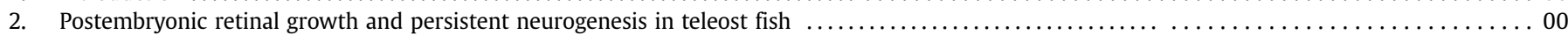

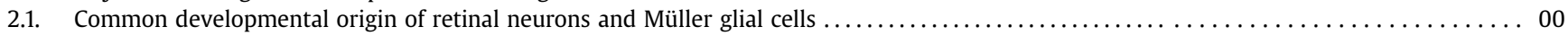

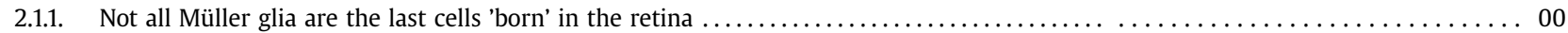

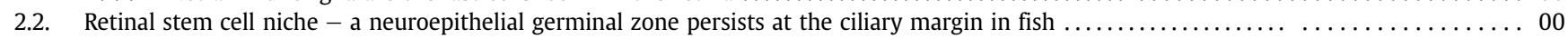

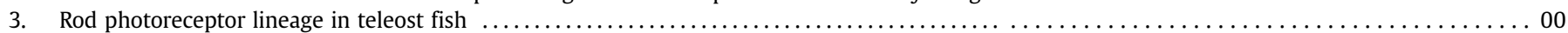

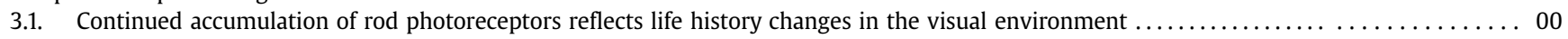

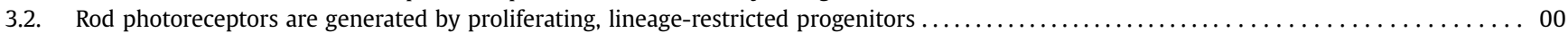

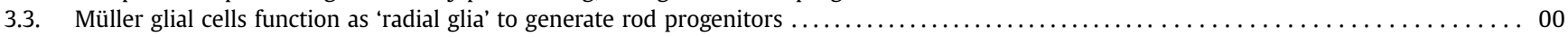

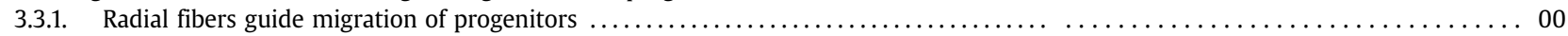

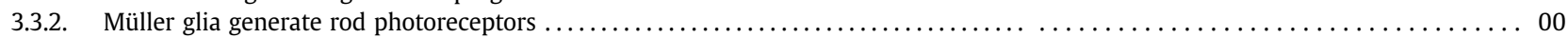

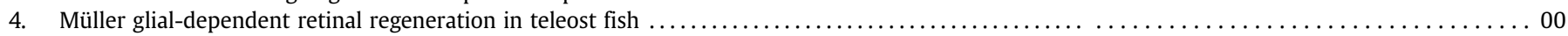

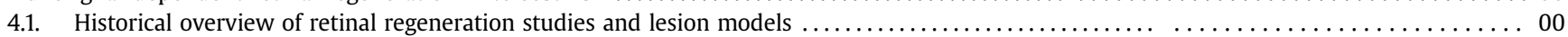

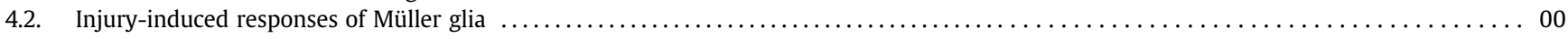

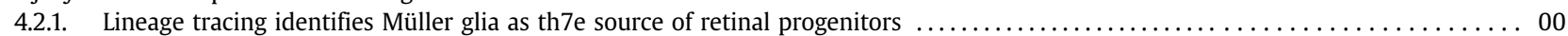

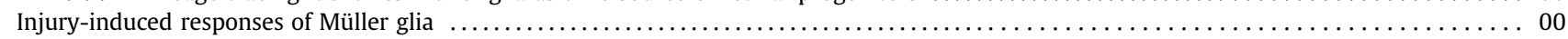

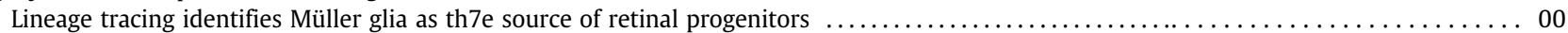

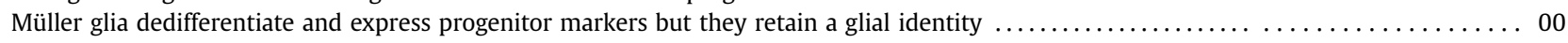

Regeneration 'niches' - neurogenic clusters derive from a self-renewing mitotic division of a Müller glia $\ldots \ldots \ldots \ldots \ldots \ldots \ldots \ldots \ldots . \ldots 0 . \ldots \ldots$

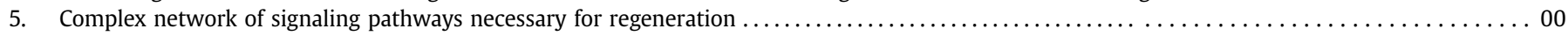

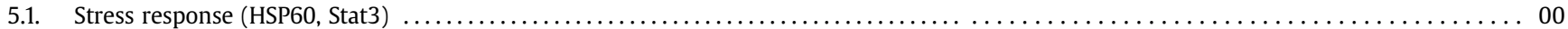

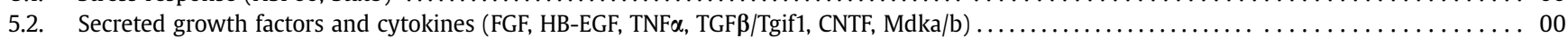

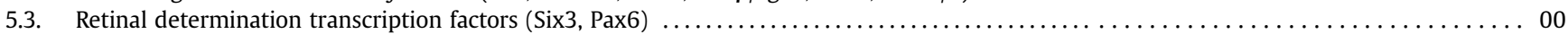

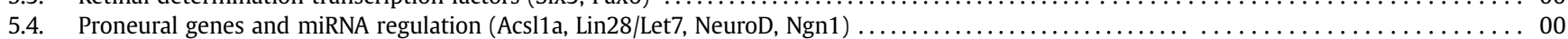

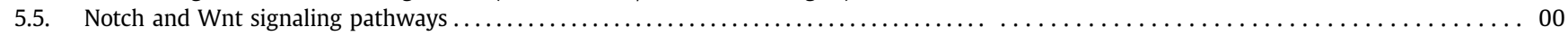

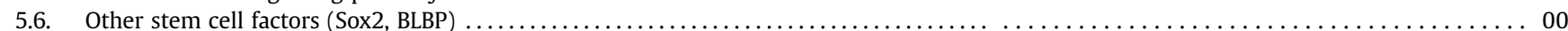

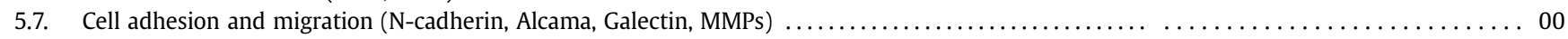

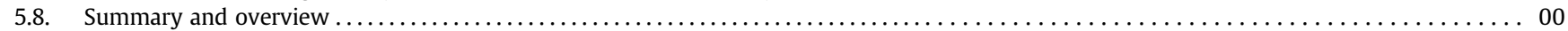

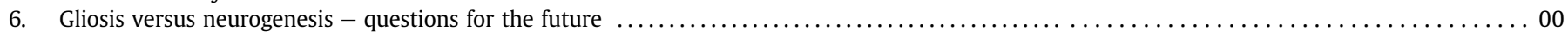

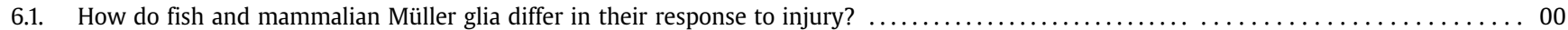

6.2. What cellular and molecular signals activate a stem-cell-like (i.e., asymmetric self-renewing) division of Müller glia rather than inducing glial scar formation?

6.3. Why do the Müller glial cell and its glial-derived retinal progenitor have such different fates (i.e., the former retains its glial properties and exits

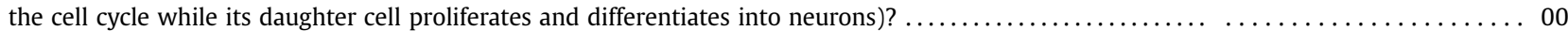

6.4. How does the physical microenvironment of Müller glia, in particular cell-cell adhesion and cell signaling as understood in the context of a stem

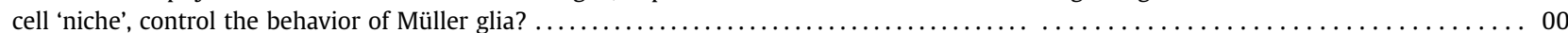

6.5. Is 'reprogramming' of Müller glia an accurate description of the regenerative response, and should this be the target for therapeutic approaches? . . 00

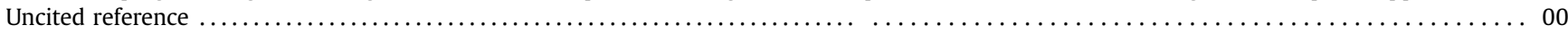

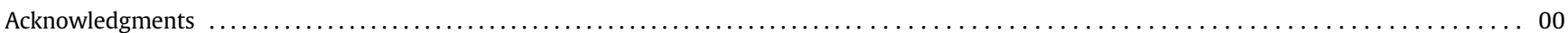

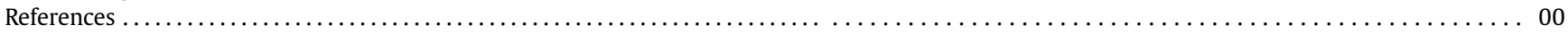

\section{Introduction}

All cells in a multicellular organism are clonally related since, by definition, they derive from a common ancestor - the single-cell zygote. The challenge of development is to generate additional cells by mitotic division and then direct those cells to exit the cell cycle and select among a multitude of distinct differentiation pathways, all within a tightly regulated spatiotemporal sequence necessary to produce and maintain the complex, multifunctional tissues and organs of the mature organism. Exactly how this myriad array of cellular interactions, complex programs of gene expression, and patterns of organogenesis are coordinated and integrated have generated questions that have intrigued students of developmental biology for many centuries. Even more complex and fascinating is the phenomenon called 'epimorphic' regeneration, which is the restoration of damaged tissues, organs, or body parts in the adult organism. The scientific revolution that was initiated by the arrival on the scene of embryonic stem cells (ESCs), somatic or 'adult' stem cells, and more recently induced pluripotent stem cells (iPSCs), has triggered a tsunami of investigators studying the fundamental developmental questions of cell lineage, cell fate, and cell differentiation. Many of these studies are inspired by the prospect of discovering and designing novel therapeutic approaches to enhance endogenous repair and regenerative therapies for the treatment of human injury and disease.

The remarkably early appearance in the developing embryo of cells fated to become the central nervous system (CNS) is a crucial event in establishing the vertebrate body plan, as first demonstrated by the pioneering work of Hans Spemann in the early 20th century. The CNS derives embryonically from a tubular sheet of pseudostratified, multipotent, proliferating neuroepithelial cells with distinct apical-basal polarity (Gotz and Huttner, 2005). Neuroepithelial cells are self-renewing and multipotent; they generate all the intrinsic cells of the CNS, neurons and glia, and are thereby often referred to as neural stem cells (Alvarez-Buylla et al., 2001; Merkle and Alvarez-Buylla, 2006; Revishchin et al., 2008; Temple, 2001). A common theme across all regions of the embryonic CNS is that neurons are generated before glial cells (Miller and Gauthier, 2007; Okano and Temple, 2009), although this characterization is not completely accurate, a caveat that is key to understanding neurogenesis and regeneration in the adult CNS. After the initial stages of neurogenesis in some regions of the brain, e.g., the 
mammalian cerebral cortex, the properties of neuroepithelial stem cells change; although they retain their distinctive spindle-shape, with apical (ventricular) and basal (pial) processes that span the width of the neuroepithelium, some neuroepithelial cells begin to express glial-specific markers, and at this stage they are identified as radial glia (Gotz and Huttner, 2005; Rakic, 2003). Radial glia continue to divide, sometimes symmetrically to expand their population, but sometimes asymmetrically, in which one daughter retains the properties of a radial glia - self-renewal - and the other becomes a neuronal progenitor that subsequently divides to generate late-born neurons; eventually radial glia in the embryonic mammalian brain differentiate into astrocytes (Campbell and Gotz, 2002; Fishell and Kriegstein, 2003; Mori et al., 2005; Morrens et al., 2012).

The discovery of the glial origin of later-born neurons in the embryonic brain overturned a long-standing dogma, which dated from studies at the end of the 19th century by Wilhelm His, who proposed separate lineages for glia and neurons from dedicated progenitors. Another dogma fell with the demonstration that neural stem cells persist in highly restricted, specialized 'niches' in the adult mammalian brain that remain in contact with the ventricles (Doetsch, 2003). It is now widely accepted that adult neural stem cells are the direct descendants of radial glia that have been sequestered and prevented from fully differentiating (AlvarezBuylla et al., 2001; Cerveny et al., 2010; Doetsch, 2003; Fuentealba et al., 2012; Kriegstein and Alvarez-Buylla, 2009; Temple, 2001). The radial glial origin of adult neural stem cells thus provides a continuous link between the limited and spatially restricted neurogenesis in the adult brain and the final stages of neurogenesis in the developing mammalian brain.

Most species of fishes grow continuously, so their body size increases throughout adult life (Brown, 1957). In contrast to the limited neurogenesis found in the adult mammal brain, neurogenesis is widespread and robust in several species of teleost fishes that have been examined (Adolf et al., 2006; Cerveny et al., 2012; Chapouton et al., 2007; Grandel and Brand, 2013; Grandel et al., 2006; Marcus et al., 1999; Zupanc, 2001, 2006; Zupanc et al., 2005). Progenitor cells in the adult teleost CNS are heterogeneous in nature, and at least two distinct populations of multipotent, proliferating cells have been described: 1) radial glial cells that express characteristic markers, e.g., glial fibrillary acidic protein (GFAP), glutamine synthetase (GS), vimentin (an intermediate filament protein), S100B (a calcium-binding protein), aromatase-B (a cytochrome P450 enzyme involved in lipid synthesis), and brain lipid binding protein (BLBP), and 2) glial-marker negative progenitors that instead express markers of neuroepithelial cells, e.g., nestin (an intermediate filament protein), Sox2 (an SRY-box transcription factor), Musashi-1 (an RNA-binding protein), and exhibit 'interkinetic nuclear migration', a distinctive property of neuroepithelial cells discussed below (Chapouton et al., 2007; Kizil et al., 2012). Persistent neurogenesis in the adult brain is associated with a robust capacity to regenerate neurons and neuronal circuits following CNS injury (Becker and Becker, 2008; Chapouton et al. 2007; Kizil et al., 2012; Zupanc, 2001, 2006; Zupanc and Sirbulescu, 2011).

The neural retina is an embryonic derivative of the brain, and as elsewhere in the CNS, retinal neurons and glia derive from multipotent neuroepithelial progenitor cells. In fish, persistent neurogenesis and the capacity to regenerate neurons leading to repair and restoration of function in damaged retinal tissue of adult animals has been recognized for decades (Easter, 1983; Hitchcock et al., 2004; Hitchcock and Raymond, 1992; Johns, 1981; Raymond, 1991, 2004; Raymond and Hitchcock, 1997, 2000). Most recent studies of persistent neurogenesis and regeneration in the retina have used the zebrafish (Danio rerio, formerly Brachydanio rerio) as an experimental model because of the many, well known advantages of this species: ease of maintaining and breeding, availability of genetic tools including mutants and transgenic lines, and a rapidly growing community of zebrafish researchers (Avanesov and Malicki, 2004; Easter and Malicki, 2002; Goldsmith and Harris, 2003; Link and Darland, 2001). Several recent reviews provide excellent summaries of our current understanding of the genetic and molecular mechanisms involved in retinal neurogenesis in zebrafish (Avanesov and Malicki, 2004; Cerveny et al., 2012; Fadool and Dowling, 2008; Stenkamp, 2007, 2011).

Studies of retinal regeneration in zebrafish are now focused squarely on Müller glial cells, since lineage tracing studies a few years ago definitively identified them as the source of retinal progenitor cells responsible for regenerating retinal neurons in the damaged retina, as we will discuss below. What has piqued the interest of many investigators, and stimulated wider interest in understanding the regenerative abilities of the fish retina, is that Müller glia in both chick and mammalian (including human) retinas display some molecular properties of retinal neuroepithelial progenitor cells and under certain conditions can give rise to cells with neuronal markers (Ahmad et al., 2011; Fischer and Bongini, 2010; Fischer and Reh, 2003; Jadhav et al., 2009; Karl and Reh, 2010; Lamba et al., 2009b; Moshiri et al., 2004; Reichenbach and Bringmann, 2013; Takeda et al., 2008). However, except in fishes, neuronal regeneration from an endogenous source of stem cells that leads to functional repair of damaged retinas has not been demonstrated. The scientific community has embraced the idea that the Müller glial origin of neuronal progenitors in the regenerating zebrafish retina is conceptually aligned with the concept of reprogramming differentiated cells into pluripotent stem cells, a discovery that has generated much excitement and captured the attention of scientists, physicians, and the public because of its promise for regenerative medicine (Hochedlinger and Plath, 2009; Jopling et al., 2011; Karl and Reh, 2010; Knapp and Tanaka, 2012; Plath and Lowry, 2011).

This review chronicles the scientific studies that led to our present understanding of the neurogenic properties and stem celllike behavior of Müller glial cells in teleost fish retinas. We conclude by posing a series of unanswered questions in the hope of stimulating additional investigations that will lead eventually to therapies designed to unlock and enhance the latent ability of human Müller glial cells to generate new retinal neurons.

\section{Postembryonic retinal growth and persistent neurogenesis in teleost fish}

\subsection{Common developmental origin of retinal neurons and Müller glial cells}

The structure and function of the vertebrate retina has been remarkably conserved over more than 500 million years of evolution, and this is reflected in a common developmental program. To briefly summarize, at an early stage in embryonic development, bilaterally paired optic primordia, which express specific 'eye field' transcription factors, evidence of commitment to a retinal fate (Chuang and Raymond, 2002; Zuber et al., 2003), grow out laterally from the forebrain to reach the surface ectoderm, then invaginate to form the optic cup. The inner surface of the cup develops into the neural (sensory) retina and the outer surface becomes the retinal pigmented epithelium (RPE); the apical surfaces of the neural and pigmented epithelia appose each other, sandwiched between the collapsed bilayers of the optic cup (Chow and Lang, 2001). The retinal neuroepithelium is a pseudostratified, columnar epithelium similar to the rest of the embryonic CNS. Retinal neuroepithelial cells are spindle-shaped with radial processes that span the width 
of the epithelium, from the apical surface (outer limiting membrane, equivalent to the ventricle in the brain) to the basal surface (inner limiting membrane, equivalent to the pia mater in the brain) (Fig. 1) (Ramón y Cajal, 1960).

The nuclei of proliferating neuroepithelial cells exhibit the peculiar property 'interkinetic nuclear migration', which is associated with stages of the cell cycle (Fujita and HoriM, 1963; Hinds and Hinds, 1974; Sauer, 1935): DNA synthesis occurs when the nucleus is located more basally in the epithelium, the nucleus then translocates apically within the radial process, and mitosis is at the apical surface. Although these original descriptions of interkinetic nuclear migration (IKNM) suggested that the basal process is withdrawn and the cell rounds up to divide, more recent time-lapse studies with fluorescent markers show that during mitosis of radial glia in the mouse cortex (Noctor et al., 2001) or neuroepithelial cells in the embryonic zebrafish retina (Baye and Link, 2007; Das et al., 2003; Del Bene et al., 2008; Norden et al., 2009) the basal process of at least one of the two daughter cells is retained. Thus, neuroepithelial cells in the developing retina have at least two distinct roles: 1 ) generate retinal cells, and 2) provide a vertical scaffolding around which neural circuits are built. After a phase of rapid proliferation and expansion of the retinal neuroepithelium, histogenesis of the neural retina commences (Fujita and HoriM, 1963; Hinds, 1968; Hinds and Hinds, 1974, 1978; Hollyfield, 1972, 1968; Kahn, 1974; Mann, 1928; Ramón y Cajal, 1960; Rapaport et al., 1996, 2004; Sidman, 1960).

Retinal histogenesis is largely a process of sequential delamination of the neuroepithelium to generate the mature neural retina, which is a remarkably ordered, laminar arrangement of six fundamental types of retinal neurons parceled into three nuclear layers and supported by one type of endogenous glial cell, the Müller cell, which was first described by Heinrich Müller in the mid-19th century. Lineage-tracing studies have convincingly shown that retinal neurons and Müller glia have a common origin individual retinal neuroepithelial cells are multipotent and form radially distributed clones of retinal cells (Holt et al., 1988; Turner and Cepko, 1987; Turner et al., 1990; Wetts and Fraser, 1988). In all vertebrate retinas, photoreceptors in the outer nuclear layer (ONL) are sensory neurons that retain many apical features of epithelial cells, including adherens junctions at the apical surface (outer limiting membrane, OLM) and an elaborate, cilium-derived appendage that contains the molecular machinery of visual transduction. The axons of photoreceptors synapse on interneurons in the inner nuclear layer (INL), which in turn provide input to retinal

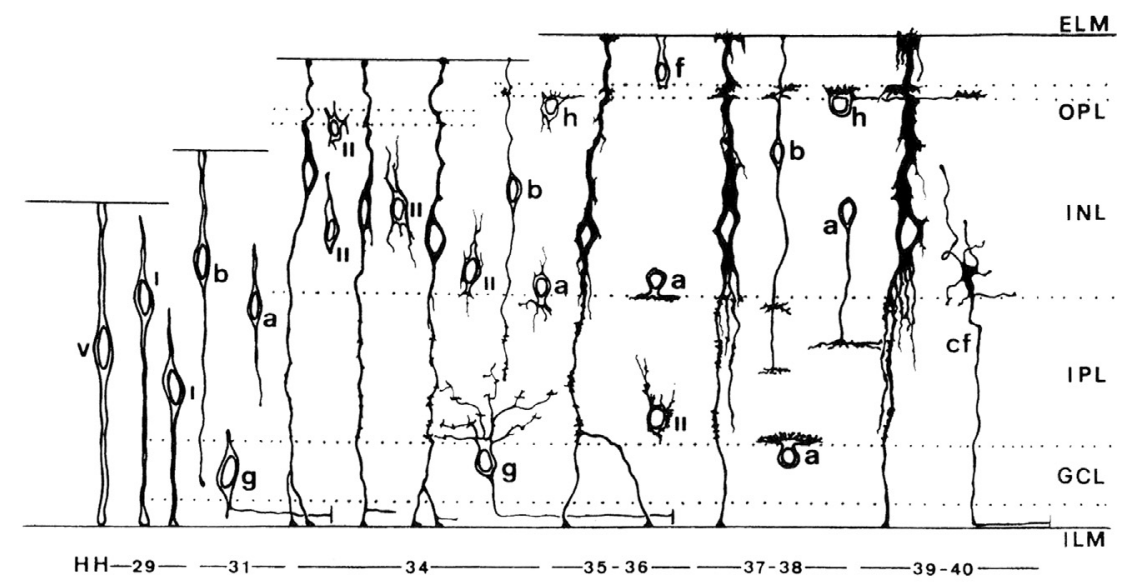

53

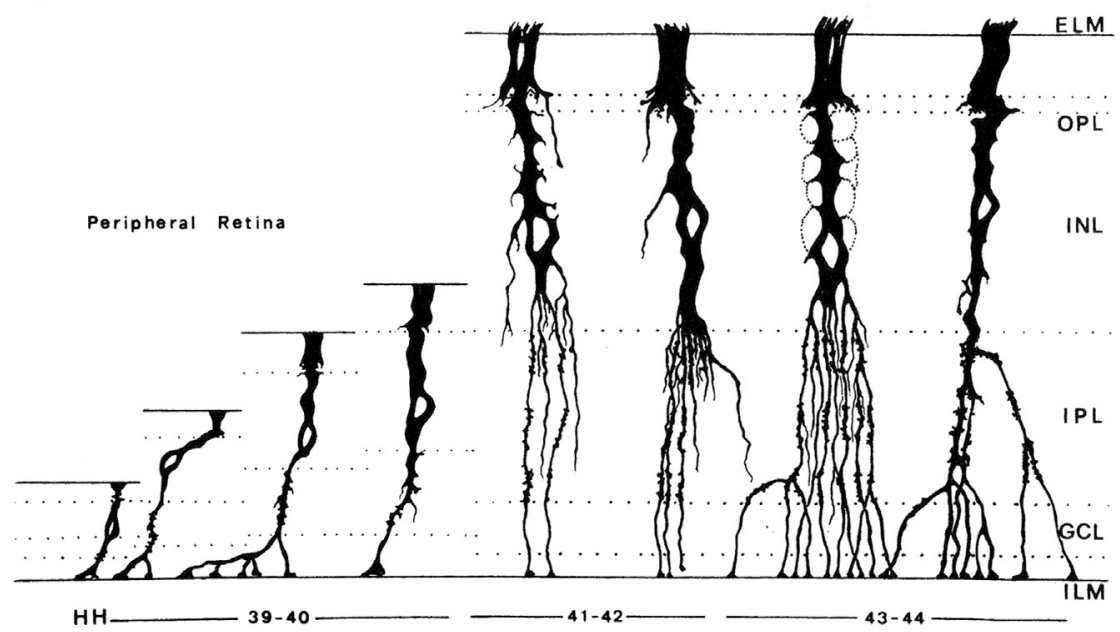

54

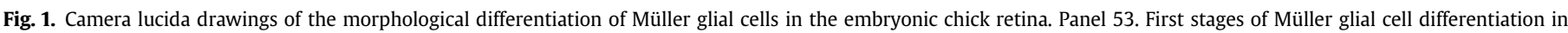

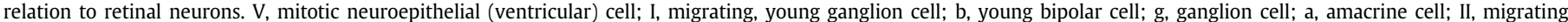

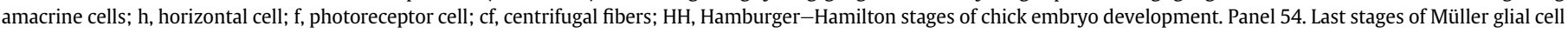
differentiation showing the central-peripheral gradient of differentiation. Reprinted with permission from Prada et al., 1989. 
ganglion cells in the innermost ganglion cell layer (GCL), whose axons exit the eye as the optic nerve.

Müller cells are specialized radial glia with nuclei in the INL and a radial process that extends apically and basally to span the width of the retinal epithelium to provide structural and metabolic support for retinal neurons (Bringmann et al., 2006; Reichenbach and Bringmann, 2013; Sarthy and Ripps, 2001). The diverse roles of Müller glia include: 1) They have profuse, elaborate lateral projections that ensheath the cell bodies and processes of every retinal neuron. 2) They provide homeostatic regulation of extracellular volume and composition (e.g., water, nutrients, ions, $\mathrm{pH}$, waste products, and trophic factors). 3) They regulate blood flow and maintain the blood-retinal barrier. 4) They support synaptic activity of neurons. 5) They function as light-guides and mediate mechanical tissue homeostasis. 6) They contribute to the isomerization of retinoids in the cone visual cycle. 7) Finally, they become reactive/activated after a pathological disturbance of the retina, and this response can have both positive (neuroprotective) and/or negative (gliosis, glial scar) consequences.

\subsubsection{Not all Müller glia are the last cells 'born' in the retina}

In the late 19th century, Santiago Ramón y Cajal made astonishingly astute observations of neurons and glial cells in mature and developing CNS tissues from several vertebrate species that were prepared with the Golgi method and other stains, and he concluded that Müller glia are among the earliest retinal cells to show signs of differentiation (Ramón y Cajal, 1972). This conclusion has been supported by a number of more recent studies of the developing retina with electron microscopy, histochemistry, and Golgi staining. Distinctive ultrastructural features of early differentiating Müller glia in the prenatal rabbit (Uga and Smelser, 1973) and rat (Kuwabara and Weidman, 1974) retinas include abundant smooth ER and lipid droplets, and the appearance of esterase activity identifies Müller glia in prenatal mouse retina (Bhattacharjee and Sanyal, 1975). Immunocytochemical staining for the Müller glial marker vimentin reveals early differentiating Müller glia in chick embryo retinas (Lemmon and Rieser, 1983), and Golgi staining in the developing chick retina also documents early morphological differentiation of Müller glia, in particular, the initial formation of lateral, cytoplasmic projections that enwrap the neurons (Prada et al., 1989). Together these studies suggest that during retinal histogenesis, some neuroepithelial cells gradually differentiate into Müller glia while retinal neurons are being generated (Fig. 1).

The morphological evidence for early differentiation of Müller glia is contrary to the current widely held belief that Müller glia are among the last cells generated during retinal development, a proposition that is based on 'birthdating' studies (Sarthy and Ripps, 2001). Beginning with the pioneering study by Richard Sidman (Sidman, 1960), numerous investigators have used a pulse of ${ }^{3} \mathrm{H}-$ thymidine to mark retinal progenitor cells synthesizing DNA in preparation for mitotic division (Fujita and HoriM, 1963; Hollyfield, 1972; Kahn, 1974; LaVail et al., 1991; Rapaport et al., 2004; Stiemke and Hollyfield, 1995; Young, 1985a, b). Because daughter cells that become postmitotic and differentiate permanently retain the radioactive label, whereas proliferating cells that undergo further mitotic divisions dilute out the label, the time of exposure to ${ }^{3} \mathrm{H}-$ thymidine indicates the terminal mitotic division of a cell - its 'birthdate'. This method works well for terminally mitotic cells, such as neurons, but since Müller glia reenter the cell cycle under certain conditions, if they do so in the developing retina, then early differentiating Müller glia that are still mitotically active would be missed using this ${ }^{3} \mathrm{H}$-thymidine-birthdating strategy.

Several studies suggest that differentiating Müller glia in the embryonic retina are not terminally mitotic, but continue to proliferate similar to radial glia in the brain. For example, in the developing cat retina, while proliferating neuroepithelial cells were still dividing at the OLM, a few mitotic cells in the INL that incorporated ${ }^{3} \mathrm{H}$-thymidine were thought to be Müller glia (Rapaport et al., 1984). Similarly, in frogs (Xenopus and Rana), scattered cells in the INL with elongated nuclei characteristic of Müller glia incorporate ${ }^{3} \mathrm{H}$-thymidine after retinal lamination (Hollyfield, 1968; Hollyfield, 1971; Stiemke and Hollyfield, 1995; Straznicky and Gaze, 1971). None of these studies used Müller-specific markers, so the identity of the proliferating cells was not certain. A study of Müller cell differentiation in zebrafish embryos noted that Müller-specific markers such as GS appeared at 60 hours postfertilization (hpf), and since most cells in the INL become postmitotic at $54 \mathrm{hpf}$ (Hu and Easter, 1999), they concluded that Müller glia were postmitotic (Peterson et al., 2001). However, subsequent studies provided evidence that Müller glia in zebrafish embryos do proliferate after they begin to differentiate. In transgenic zebrafish with a Müller glial-specific reporter - $\operatorname{Tg}$ (gfap:EGFP), in which expression of enhanced green fluorescent protein (GFP) is driven by regulatory elements of the glial fibrillary acidic protein ( $g$ fap) gene (Bernardos and Raymond, 2006) - GFP-labeled Müller glia appear in the embryonic retina as early as $48 \mathrm{hpf}$, and they are mitotically active (Bernardos et al., 2007). Similarly, Müller glia identified in zebrafish embryos by immunolabeling with anti-GS or anti-GFAP incorporated BrdU at 55 and $60 \mathrm{hpf}$ (Nelson et al., 2008).

Despite these contradictory reports in the literature about when Müller glia are 'born' in the developing retina, since Müller glia are critical to the structural integrity of the laminated retina and stabilize the vertical columns of neurons (Layer and Willbold, 1993; Willbold et al., 1995), it would not be surprising that when neurons start to differentiate they quickly begin to require the supportive functions of Müller glia. Furthermore, there is no dispute about the ability of differentiated Müller glia from the adult retina to proliferate under pathological conditions or in vitro (Bringmann et al., 2003, 2006; Dyer and Cepko, 2000a; Fisher and Lewis, 2003; Sarthy, 1985, 1991).

In all vertebrates, two general patterns of retinal differentiation are observed (Mann, 1928; Ramón y Cajal, 1960). First, retinal ganglion cells near the center of the hemispheric optic cup adjacent to the optic stalk are the first to differentiate. Second, gradients of differentiation then progress from inner to outer layers and from center to periphery of the retinal hemisphere. As a result of these two development patterns: 1 ) rod photoreceptors are the last type of neurons generated (inner-to-outer gradient), and 2) the last stages of neurogenesis are at the peripheral margin of the retina, at the boundary with the ciliary epithelium (central-to-peripheral gradient). The consequences of these ontogenetic patterns of retinal development are discussed next.

\subsection{Retinal stem cell niche - a neuroepithelial germinal zone persists at the ciliary margin in fish}

As fishes grow during larval and adult life, the retina enlarges by a combination of intraocular expansion and cellular hypertrophy as well as neurogenesis (Ali, 1964; Fernald, 1991; Johns, 1977, 1981; Johns and Easter, 1977; Lyall, 1957; Meyer, 1978; Müller, 1952; Sandy and Blaxter, 1980). The increase in retinal size and rate of neurogenesis is variable with age and among individuals (Brown, 1957) and is coordinated with body growth at least in part through hormonal regulation mediated by the growth hormone/ IGF-1 axis (Boucher and Hitchcock, 1998; Mack and Fernald, 1993; Otteson et al., 2002; Otteson and Hitchcock, 2003). The neurons that contribute to the increase in retinal size are largely born in the circumferential germinal zone at the ciliary margin where neuroepithelial cells generate concentric annuli of new retinal tissue (Amato et al., 2004; Centanin et al., 2011; Cerveny 
et al., 2012; Harris and Perron, 1998; Hitchcock et al., 2004; Hitchcock and Raymond, 2004; Moshiri et al., 2004; Otteson and Hitchcock, 2003; Raymond et al., 2006; Stenkamp, 2007). The sequence of histogenesis in the newly generated retina at the periphery recapitulates embryonic and larval stages of retinal development, including the order of generation of different cell types. In fact, the vast majority of the neural retina in adult fish (and frogs) is generated postembryonically by neurogenesis in the circumferential germinal zone, or ciliary marginal zone (CMZ) (Allison et al., 2010; Moshiri et al., 2004; Raymond, 1986). In contrast, limited neurogenesis occurs in the CMZ of early postnatal birds, but in mammals the CMZ is absent (Kubota et al., 2002); an exception is that in mice heterozygous for a null mutation in patched (ptc) - the receptor for Shh (Sonic hedgehog), a mitogenic factor for retinal progenitors in vitro - proliferating retinal progenitors are present in the CMZ, and neurogenesis continues up to 3 months of age (Moshiri and Reh, 2004). Similarly, in zebrafish, mutations in ptc2 result in expansion of progenitors in the CMZ (Bibliowicz and Gross, 2009).

Neuroepithelial cells in the $\mathrm{CMZ}$ of fish and larval frogs include multipotent, retinal stem cells that self-renew and generate all types of retinal neurons and Müller glia (Fig. 2 and Agathocleous and Harris, 2009; Centanin et al., 2011; Raymond et al., 2006; Wehman et al., 2005). In the CMZ of both Xenopus (Harris and Perron, 1998; Xue and Harris, 2012) and zebrafish (Raymond et al., 2006), developmental stages are represented spatially, reflecting the appositional mode of cell addition, i.e., retinal stem cells are most peripheral, adjacent to the ciliary epithelium, then multipotent progenitors, and then committed retinal progenitors most central, adjacent to the differentiating retinal cells. The regulation of proliferation, gene expression profiles, and signaling pathways that characterize retinal stem and progenitor cells in the CMZ have been studied extensively (Bibliowicz and Gross, 2009; Calinescu et al., 2009; Cerveny et al., 2010; El Yakoubi et al., 2012; Gonzalez-Nunez et al., 2010; Kubo and Nakagawa, 2009; Kubo et al., 2003, 2005; Meyers et al., 2012; Moshiri et al., 2004, 2005; Negishi et al., 1990; Perron and Harris, 2000; Raymond et al., 2006; Reh and Levine, 1998; Stenkamp, 2007; Stenkamp et al., 1997; Stephens et al., 2010; Vitorino et al., 2009; Xue and Harris, 2012).
The relevant signaling pathways include IGF (Insulin-like Growth Factor), FGF (Fibroblast Growth Factor), Hedgehog, Wnt (Winglesstype MMTV integration site family), BMP (Bone Morphogenetic Protein), retinoic acid, thyroid hormone, and Notch. Transcription factors associated with specification of retinal cell fate and expressed in the CMZ include homeobox genes $r \times 1 / 2$ (retinal homeobox 1/2), pax6 (paired homeobox 6), six3 (sine oculis homeobox 3), vsx2 (visual system homeobox 2), and proneural genes ascl1a (achaete-scute complex homolog 1a), neurod (neurogenic differentiation), atoh7 (atonal homolog 7), among others.

The CMZ and surrounding microenvironment in adult zebrafish retina (Fig. 2) share many characteristics with neural stem cell niches in the adult mammalian brain: 1) Radial-glial markers such as BLBP (also known as fatty acid binding protein and encoded by the zebrafish gene, $f a b p 7 a$ ) are expressed by retinal stem and progenitor cells; 2) The extracellular-matrix associated carbohydrate epitope SSEA-1/LeX/CD15 is expressed on the circumferential blood vessel that lies on the vitreal surface adjacent to the $\mathrm{CMZ}$ and on the overlying RPE; 3) N-cadherin protein (encoded by the cdh2 gene) is diffusely distributed on the basolateral plasma membranes of retinal stem and progenitor cells as well as localized to specialized adherens junctions at the OLM (Raymond et al., 2006).

The stem cell niche in the $\mathrm{CMZ}$ represents a remnant of the last stages of embryonic retinal development - retinal stem and progenitor cells that have resisted the wave of differentiation that swept from center of the retina to the peripheral margin. The second developmental gradient builds the retinal laminae from the inner surface (retinal ganglion cells) to the outer surface (rod photoreceptors). In teleost fish retinas, the second development gradient also extends into adult life, with the persistent generation of rod photoreceptors, as discussed next.

\section{Rod photoreceptor lineage in teleost fish}

\subsection{Continued accumulation of rod photoreceptors reflects life history changes in the visual environment}

In some species of teleost fishes, the retina has cones but few or no rods during larval stages, and rods begin to appear in the

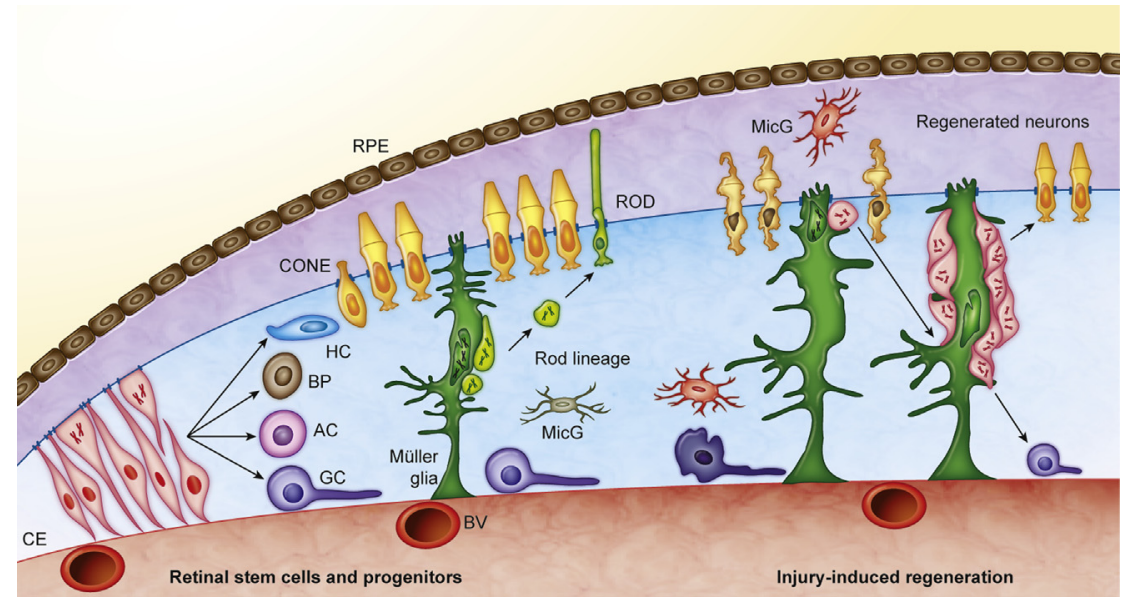

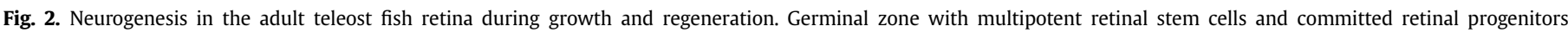

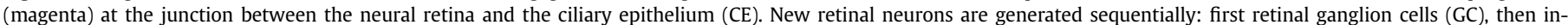

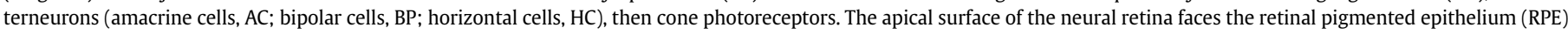

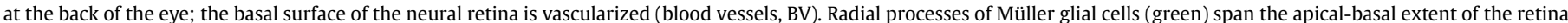

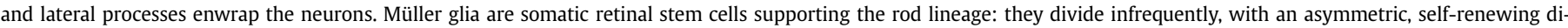

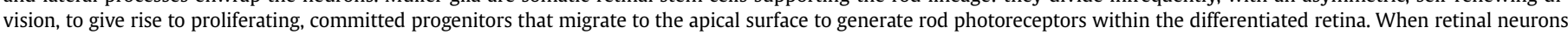

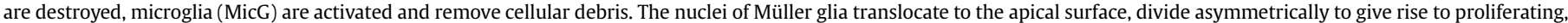

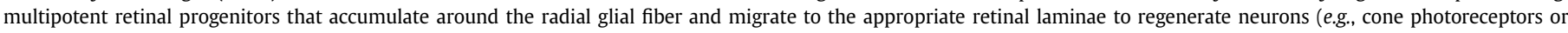
retinal ganglion cells). 
differentiated retina only during larval development or at metamorphosis (Blaxter, 1975; Blaxter and Staines, 1970), and subsequently rods continue to increase in proportion to other retinal cells during post-larval retinal growth (Branchek and Bremiller, 1984; Johns and Easter, 1977; Kunz et al., 1983; Locket, 1980; Lyall, 1957; Müller, 1952; Raymond, 1985; Stenkamp et al., 1997, 1996). The delayed appearance of rod photoreceptors in the retina has been attributed to changes in the life history and photic environment of fishes: The larvae live near the surface of the water, where sunlight penetrates, but as they grow larger they move deeper, ambient light diminishes and rods become more important to visual function. The first thorough quantification of the differential addition of rods to the retina was a study published in 1952 by Hans Müller, who counted retinal cells and calculated total numbers in guppies (Lebistes reticulatus) from hatching to adult stages; he found a three-fold increase in ganglion cells and cones, but a sevenfold increase in rods (Müller, 1952). Similarly, in adult goldfish (Carassius auratus) from 1 to 4 years of age, the number of ganglion cells and cones increases almost six-fold, but the number of rods increases ten-fold (Johns and Easter, 1977). The accumulation of rods in the adult goldfish retina has two consequences: 1) The planimetric density of rods (number per $\mathrm{mm}^{2}$ of retina surface) increases slightly with growth, whereas the density of other retinal neurons decreases as a result of retinal stretching (Johns and Easter; 1977); 2) the dark-adapted visual sensitivity, as measured psychophysically, reflects the slight increase in rod density (Powers et al., 1988).

The delayed generation of rod photoreceptors compared with cone photoreceptors is not specific to fishes. In mammalian retinas, cones are also generated before rods, and in most species production of rods continues postnatally (Carter-Dawson and LaVail, 1979; Rapaport et al., 1996, 2004; Turner and Cepko, 1987; Turner et al., 1990; Young, 1985a). We next turn to a discussion of the retinal progenitors that generate rod photoreceptors within the fully differentiated, functioning retina of adult fishes.

\subsection{Rod photoreceptors are generated by proliferating, lineage- restricted progenitors}

The rod photoreceptors that accumulate in the adult fish retina are not born in the $\mathrm{CMZ}$ but derive from proliferating rod precursors, which appear to be lineage-restricted progenitors (Fig. 2). Inga-Britt Ahlbert observed mitotic figures in the ONL of larval salmonid (Salmo) species and suggested that they might be generating rods (Ahlbert, 1976); similar observations had been made earlier in other fish species (Lyall, 1957; Vilter and Lewin, 1954). This suggestion was confirmed in the 1980's when proliferating cells in the ONL that incorporated ${ }^{3} \mathrm{H}$-thymidine and then differentiated into rods were discovered in metamorphosing herring (Clupea harengus) and sole (Solea solea) (Sandy and Blaxter, 1980), and juvenile cichlids (Haplochromis burtoni) and goldfish (Johns and Fernald, 1981). Follow-up longitudinal studies of ${ }^{3} \mathrm{H}-$ thymidine-labeled cells in embryonic cichlids (Hagedorn and Fernald, 1992) and larval goldfish (Johns, 1982), in which production of rods occurs at a high rate, revealed that retinal progenitors in the INL incorporate ${ }^{3} \mathrm{H}$-thymidine and form migrating chains of elongated, progenitor cells that move apically into the ONL, where they continue to proliferate before differentiating into rod photoreceptors (Fig. 3 ). The newly generated, ${ }^{3} \mathrm{H}$-thymidine-labeled rod photoreceptors in goldfish persist for at least 180 days (Johns, 1982), so rod genesis is not likely to reflect a process of photoreceptor turnover. The addition of rod photoreceptors is especially evident in the peripheral retina, which is most recently generated by the CMZ and therefore still actively acquiring its cohort of rod photoreceptors.
Although a pulse of ${ }^{3} \mathrm{H}$-thymidine delivered to adult goldfish labels the mitotically active rod precursors in the ONL, this technique often labels few if any proliferating progenitors in the INL. Subsequent studies used continuous exposure (for one to several days) to the thymidine analog bromodeoxyuridine (BrdU) and observed BrdU-labeled progenitors in the INL of juvenile and adult rainbow trout (Onchorynchus mykiss) (Julian et al., 1998) and goldfish (Otteson et al., 2001) retinas. The implication from these observations is that the INL progenitors are quiescent or slowly cycling, and exposure to a brief pulse of BrdU (or ${ }^{3} \mathrm{H}$-thymidine) is therefore unlikely to be synchronous with the S-phase of the cell cycle. Juan Korenbrot and colleagues used a double-labeling technique with sequential exposure first to BrdU then to iododeoxyuridine (IdU) to demonstrate that rod precursors in the ONL are derived from INL progenitors that form clusters in the INL and migrate into the ONL (Julian et al., 1998). After systemic exposure of juvenile goldfish to BrdU for 9 days, Peter Hitchcock and colleagues also found many BrdU+ rods in the ONL at 30 days and rare BrdU+ cells in the INL that were immunoreactive for the progenitor marker Pax6; they concluded that these label-retaining cells were slowly cycling 'stem cells' at the origin of the rod photoreceptor lineage (Otteson et al., 2001). As discussed in Section 3.3 below, the INL 'stem cells' have now been identified as Müller glia.

Rod precursors in the ONL do not express Pax6 (Hitchcock et al., 1996), and because they proliferate rapidly, they are easily visualized by immunostaining with a marker for late G2/M phase, PCNA (proliferating cell nuclear antigen). In the cichlid retina, rod precursors show a circadian rhythm of proliferation with a higher number of mitoses in the dark phase (Chiu et al., 1995). Ocular growth and retinal stretching also modulate the rate of proliferation of rod precursors. In both goldfish (Raymond et al., 1988a) and zebrafish (Sherpa et al., 2011) mutant strains have been isolated that have grossly enlarged eyes due to elevated intraocular pressure (a phenotype related to congenital glaucoma in humans, or buphthalmia); in these fish the number of ${ }^{3} \mathrm{H}$-thymidine or BrdU-labeled rod precursors is increased, indicating a larger population that generates more rod photoreceptors.

Subsequent studies have provided insights into the genetic regulation of the rod lineage in zebrafish (for details, see the recent review by Deborah Stenkamp; Stenkamp, 2011). Proliferating progenitors in the rod lineage of the late embryonic to adult zebrafish originate in the INL and migrate to the ONL while undergoing progressive changes in expression of transcription factors associated with commitment to retinal and photoreceptor cell fate: pax6expressing cells in the INL give rise to migrating progenitors that express $r x 1$ and neurod; as the progenitors move into the ONL they express crx (cone-rod homeobox) and nr2e3 (nuclear receptor subfamily 2, group E, member 3; an orphan nuclear receptor known to activate rod-specific genes and repress cone-specific genes) indicative of rod photoreceptor specification (Bernardos et al., 2007, 2005; Nelson et al., 2008; Ochocinska and Hitchcock, 2007, 2009).

Several laboratories have shown that the population of proliferating rod precursors in the ONL, and thereby the rate of rod photoreceptor production in fishes, is subject to hormonal regulation associated with growth (rapidly growing fish have more proliferating rod precursors); selective destruction of rod photoreceptors also results in an increased number of proliferating rod precursors (reviewed in Stenkamp, 2011). A recently described transgenic model of rod photoreceptor degeneration in zebrafish has been especially useful for understanding the regulation of rod photoreceptor genesis (Morris et al., 2005). In this transgenic line, $\mathrm{Tg}$ (XOPS:mCFP), the Xenopus rod opsin promoter drives expression of membrane-targeted cyan fluorescent protein, which causes rods to degenerate as soon as they begin to differentiate, presumably as a result of toxic levels of the reporter protein, but cones are not 

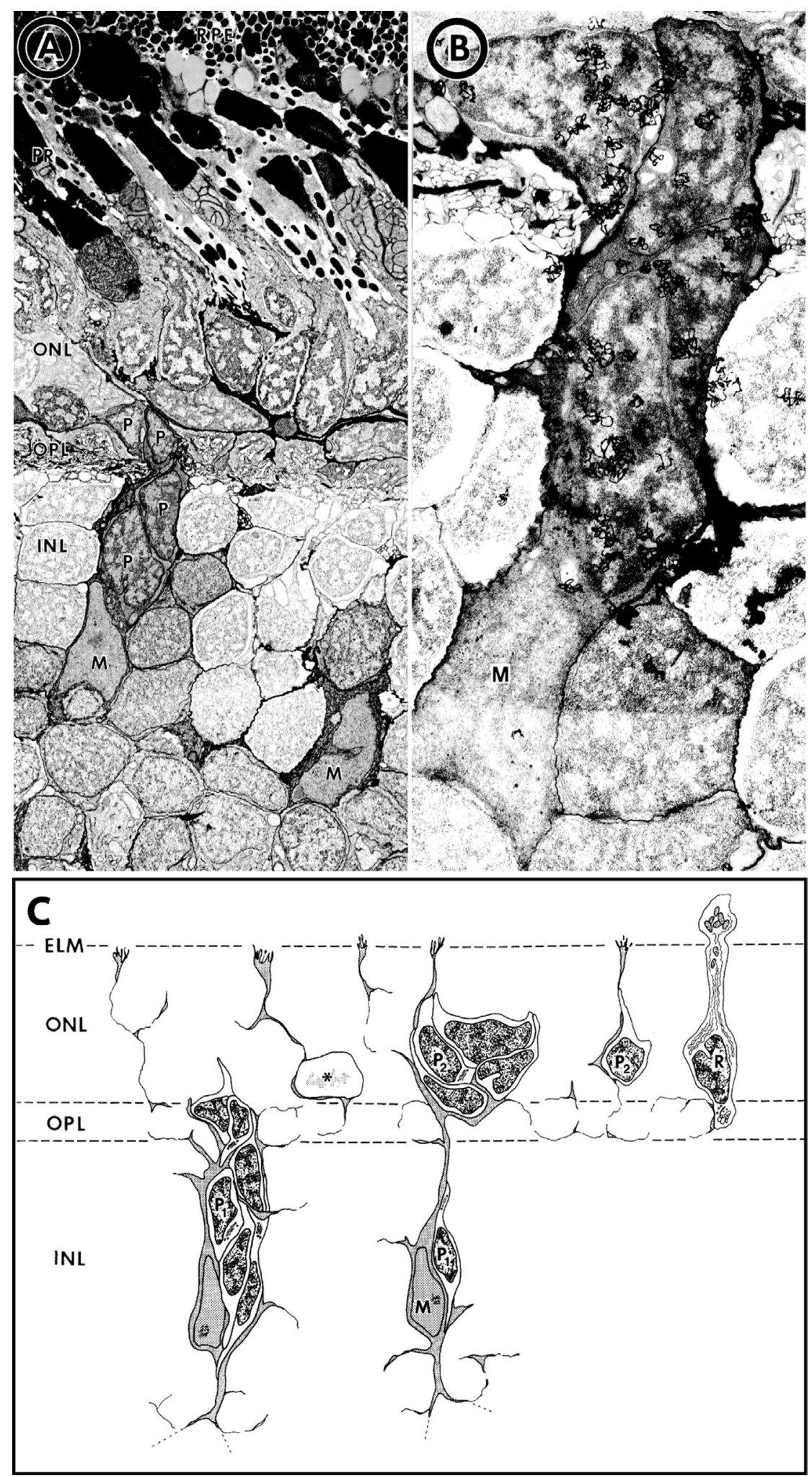

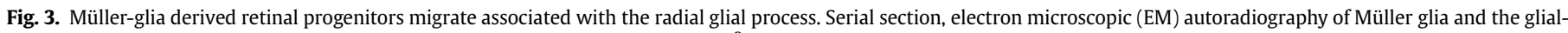

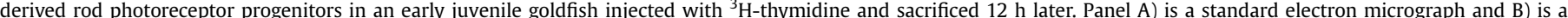

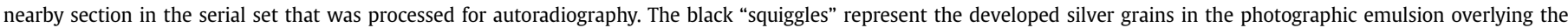

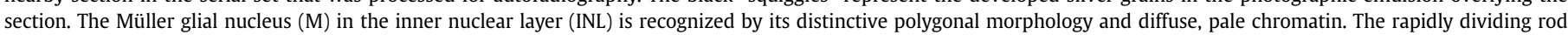

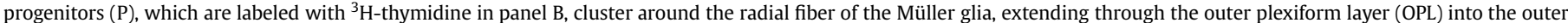

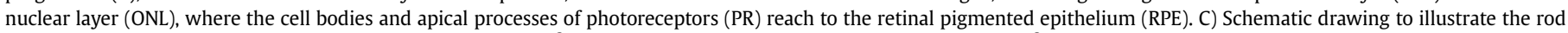

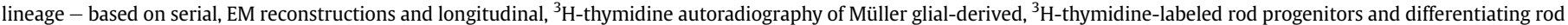
photoreceptors. Reprinted with permission from Raymond and Rivlin (1987). 
affected. In response, the population of proliferating rod precursors is expanded by almost three-fold in the adult retina (Morris et al., 2005). A microarray analysis of the transcriptome of $\mathrm{Tg}$ (XOPS:mCFP) zebrafish compared with wild-type zebrafish uncovered three transcription factors that in adults are expressed selectively in the CMZ and the proliferating rod precursors in the ONL: a member of the Sox group C family, sox $11 b$; $a$ zinc-finger transcription factor, insm1a (insulinoma-associated 1a); and a member of the myeloblastosis family of proto-oncogenes, c-myb (Morris et al., 2011). A subsequent study showed that insm1a expressed in proliferating rod precursors in the ONL is negatively regulated by Notch signaling, and promotes cell cycle progression, expression of neurod, crx, and nr2e3, and differentiation of rod photoreceptors (Forbes-Osborne et al., 2013).

The mechanisms that determine the choice of rod versus cone fate by the photoreceptor progenitors in the fish retina are not yet fully understood, but retinoic acid (RA) signaling has been implicated in rod/cone cell fate decisions (Stevens et al., 2011). Zebrafish have four morphologically, spectrally, and functionally distinct types of cones that express specific cone opsin genes, rendering them maximally sensitive to different wavelengths of light: long (red), medium (green), short (blue), and ultraviolet (UV), respectively (Chinen et al., 2003; Hisatomi et al., 1996; Raymond et al., 1993). Exposure of zebrafish embryos to exogenous RA alters the numbers of rods and cones - rod photoreceptors are increased and cones, especially red cones, are reduced - whereas morpholino-targeted knockdown of the RA nuclear receptor, RAR $\alpha \beta$, results in a decrease in number of rods (Stevens et al., 2011). Exposure to RA in vitro also biases retinal progenitor cells from embryonic and neonatal rats to develop as rod photoreceptors (Kelley et al., 1994). In the mouse retina, a transcriptional network controls photoreceptor cell fate decisions, rod versus cone (reviewed in Swaroop et al., 2010). In the mammalian retina, specification of rod fate, and concomitant suppression of cone fate, is controlled by a cascade of transcription factors, including a receptor related to RAR $\alpha \beta$ called ROR $\beta$ (RARrelated orphan nuclear receptor $\beta$ ), its downstream target NRL (Neural Retina Leucine-zipper transcription factor in the Maf subfamily), and the NRL target Nr2e3. In contrast, in zebrafish the progenitors for both rods and cones have similar molecular profiles including expression of $r \times 1, c r x$, neurod, $n r l$, and $n r 2 e 3$ (reviewed in Stenkamp, 2011), suggesting that mechanisms underlying photoreceptor commitment and cell fate selection in different vertebrate species may vary (Adler and Raymond, 2008).

\subsection{Müller glial cells function as 'radial glia' to generate rod progenitors}

\subsubsection{Radial fibers guide migration of progenitors}

The identity of the slowly cycling, Pax6+ progenitors in the INL that give rise to the rod photoreceptor lineage remained an enigma for many years (Johns, 1982; Julian et al., 1998; Otteson et al., 2001), although a hint of their origin was evident in that the clusters or chains of proliferating, spindle-shaped progenitors appeared to migrate from INL to ONL in close association with the radial processes of Müller glia (Fig. 2). This relationship was demonstrated directly by serial electron microscopic (EM) reconstruction combined with longitudinal ${ }^{3} \mathrm{H}$-thymidine autoradiography in larval goldfish retinas (Fig. 3, see Raymond and Rivlin, 1987). The ${ }^{3} \mathrm{H}$-thymidine-labeled progenitors in the INL are bipolar-shaped with short $(<200 \mu \mathrm{m})$ leading processes that curl around the radial Müller fiber and neighboring progenitors in the migrating chain. These characteristics are consistent with a migration pathway guided by the radial glial fiber, and are remarkably similar to the morphology of neuroblasts in the embryonic mammalian brain migrating along the radial glial fibers to reach the neocortex (Rakic, 1972).
For serial EM reconstructions of ${ }^{3} \mathrm{H}$-thymidine-labeled progenitors (Fig. 3), we injected a pulse of ${ }^{3} \mathrm{H}$-thymidine and then processed the retinas $1 \mathrm{~h}$ to 14 days later; we examined 235 labeled cells from 11 retinas. We could longitudinally track the label in clusters of progenitors as they migrated from the INL to ONL and then differentiated into rod photoreceptors, but we never saw a labeled Müller glial cell (Raymond and Rivlin, 1987), which in retrospect is explained by their relative quiescence in the unperturbed retina. As mentioned in the Introduction, we now know that the migrating neuroblasts in the neocortex are clonally related to the radial glial cell progenitor that guides them (Noctor et al., 2001, 2002); these studies used a retroviral vector encoding GFP to label mitotic radial glia, and examined clones up to 3 days later with live imaging and immunocytochemistry. When lineage-tracing methods were applied to developing zebrafish retinas, the INL 'stem cells' that generate rod photoreceptors were finally identified as Müller glial cells.

\subsubsection{Müller glia generate rod photoreceptors}

Lineage-tracing studies in larval zebrafish retina with transgenic Müller-glia reporter lines, $\operatorname{Tg}($ gfap:EGFP)mi2001, mi2002, showed that proliferating Müller glia are the source of rod progenitors (Bernardos et al., 2007). The cell specificity of the GFP reporter was verified by co-localization with several Müller-specific markers, including cellular retinaldehyde binding protein (CRALBP, encoded by one of the two zebrafish gene paralogs, rlbp1a), BLBP (fabp7a), and apolipoprotein E (apoE) (Bernardos and Raymond, 2006). Proliferating GFP+ Müller glia were identified by immunocytochemistry with PCNA or BrdU incorporation; approximately $4 \%$ of Müller glia in juvenile zebrafish (1.5 months old) are mitotically active, and all Müller glia are weakly immunoreactive for the retinal progenitor marker Pax6 (Bernardos et al., 2007). Continuous, systemic exposure to BrdU for 7 days revealed abundant BrdU-labeled, weakly Pax6-immunoreactive, clusters of spindle-shaped progenitors spanning the INL/ONL, some of which were also immunoreactive for the photoreceptor progenitor marker Crx. We also found several examples of GFP $+/ B r d U+$ cells in the ONL that were immunoreactive for rhodopsin, identifying them as rod photoreceptors. The neuronal progenitors that derive from mitotic divisions of Müller glia down-regulate expression of the glial marker, GFAP, so the transgene reporter is turned off, but perdurance of the GFP protein allows it to be used as a lineage tracer.

Taken together with the previous work on rod photoreceptor genesis in teleosts, the implication of this finding is that all or most of the rod photoreceptors in the fish retina are derived from differentiated Müller glial cells (Fig. 2). A possible exception is the early-differentiating 'precocious' ventronasal patch of rod photoreceptors that appears in the embryonic zebrafish retina at $\sim 50 \mathrm{hpf}$ (Kljavin, 1987; Raymond et al., 1995; Schmitt and Dowling, 1996, 1999). This has not been described in other teleost species, and its significance for retinal histogenesis or visual function is not known. The Müller glial processes in the ONL of adult zebrafish retina express abundant amounts of a modified form of NCAM (neural cell adhesion molecule), polysialic acid-NCAM (PSANCAM), associated with embryonic and adult neurogenesis, and this may provide microenvironment cues necessary for continued rod photoreceptor generation (Kustermann et al., 2010).

\section{Müller glial-dependent retinal regeneration in teleost fish}

\subsection{Historical overview of retinal regeneration studies and lesion models}

The ability to regenerate body parts and organs is a fascinating subject for scientific investigation, and understanding the 
biological mechanisms that underlie regeneration has enormous implications for the field of regenerative medicine. While some organisms can remodel remaining tissue to regenerate lost body parts, known as morphallaxis, many others rely on proliferation of dedifferentiated tissue to replace the lost tissue, known as epimorphic regeneration. Some adult urodele amphibians (newts and salamanders) and adult teleost fishes can regenerate neural retina, although the process of regeneration, and even the source of progenitor cells, varies between the two groups. Many excellent scientific reviews published in the last 10 years have described retinal regeneration in non-mammalian vertebrates (Brockerhoff and Fadool, 2011; Gallina et al., 2013; Gemberling et al., 2013; Hitchcock and Raymond, 2004; Karl and Reh, 2010; Lamba et al., 2009b; Moshiri et al., 2004; Otteson and Hitchcock, 2003; Stenkamp, 2007). Here we focus exclusively on retinal regeneration in fishes; we begin with a brief historical overview summarizing the retinal lesion paradigms that have been developed to assess the regenerative ability of the fish retina and then discuss the progress toward identifying the source of the regenerated neurons.

The first evidence that retinal neurons regenerate in teleost fish was provided by Ferdinando Lombardo $(1968,1972)$, who surgically removed the dorsal quadrant of the retina in adult goldfish $(C$. auratus) and subsequently observed mitotic figures in the $\mathrm{CMZ}$ and along the cut edges of the wound. A number of subsequent studies documented regeneration of retinal neurons, including photoreceptors, after transscleral, surgical removal of small pieces of retina in goldfish (Hitchcock, 1997; Hitchcock and Cirenza, 1994; Hitchcock et al., 1992, 1996; Hitchcock and Vanderyt, 1994), green sunfish (Leopomis cyanellus) (Cameron and Easter, 1995), zebrafish (Cameron, 2000; Cameron and Carney, 2000, 2004; Yurco and Cameron, 2005), trout (O. mykiss) (Faillace et al., 2002), and winter flounder (Pleuronectes americanus) (Mader and Cameron, 2004). The gap in the retina created by the surgery partially closes by wound contraction, and then proliferating, neuroepithelial-like retinal progenitors collect in a 'blastema' at the wound margin; eventually retinal neurons regenerate to fill in the gap and restore the cytoarchitecture. Transscleral punctures of the retina with needles in goldfish (Sullivan et al., 1997) and zebrafish (Fausett and Goldman, 2006; Liu et al., 2002) damages limited numbers of retinal neurons, but provokes a similar proliferative response at the lesion site and in surrounding tissue.

After surgical excision, retinal neurons regenerate in approximately the correct proportions and retinal circuitry is restored (Hitchcock and Cirenza, 1994; Hitchcock et al., 1992; Hitchcock and Vanderyt, 1994). In addition to the proliferative blastema at the wound margin, clusters or chains of elongated, proliferating cells in the INL appear in the undamaged retina (Cameron, 2000; Cameron and Easter, 1995; Faillace et al., 2002; Yurco and Cameron, 2005) and produce cone photoreceptors and other retinal neurons that are incorporated into the extant retina. Intraocular injections of growth factors and cytokines, such as FGF, ciliary neurotrophic factor (CNTF), and interleukin-6 (IL-6) also stimulate proliferation of cells in the INL and ONL (Faillace et al., 2002; Negishi and Shinagawa, 1993).

Hartwig Wolburg and colleagues introduced the use of neurotoxins delivered to the retina by intraocular injection; at high doses, ouabain (an irreversible inhibitor of $\mathrm{Na}-\mathrm{K}$ ATPase) completely destroys the neural retina in goldfish, whereas at lower doses, retinal neurons in the inner layers are destroyed while the photoreceptors in the outer retina survive (Maier and Wolburg, 1979). Ouabain diffuses into the neural retina from the vitreous, and neurons in the inner retina are preferentially damaged because they are exposed to a higher drug concentration (Raymond et al., 1988b). In both goldfish and trout (Kurz-Isler and Wolburg, 1982), destruction of retinal neurons by ouabain stimulates mitotic activity of retinal progenitors in the $\mathrm{CMZ}$, and the peripheral retina is restored through enhancement of the normal growth process. However, the central retina (if it is not completely destroyed by ouabain) regenerates from nests or clusters of neuroepithelial-like progenitors (Fig. 2) that are distributed across the damaged region (Kurz-Isler and Wolburg, 1982; Maier and Wolburg, 1979; Raymond et al., 1988b). Ouabain has recently been used to destroy retinal neurons in adult zebrafish (Fimbel et al., 2007; Nagashima et al., 2013), and the regenerative response is more robust compared with goldfish (Raymond et al., 1988b).

Other early studies in goldfish employed intraocular injection of more selective neurotoxins, including tunicamycin (which destroys rod photoreceptors) and 6-hydroxydopamine (which targets dopaminergic interplexiform neurons) (Braisted and Raymond, 1992, 1993; Negishi et al., 1991a,b, 1982a, 1987). Low doses of 6hydroxydopamine selectively ablate the dopaminergic cells, which are not regenerated in the central retina (Braisted and Raymond, 1992; Negishi et al., 1982b); only at higher doses that result in non-selective neuronal loss is an intrinsic regenerative response triggered in the central retina (Braisted and Raymond, 1993; Negishi et al., 1987).

Methods that preferentially destroy photoreceptors have also been developed. Argon lasers (ophthalmological instruments designed for photocoagulation) were used to focally ablate photoreceptors in small circular areas $(\sim 200-300 \mu \mathrm{m}$ diameter $)$ in goldfish retina (Braisted et al., 1994; Sullivan et al., 1997; Wu et al., 2001), and thermal probes were used in zebrafish (Raymond et al., 2006). Most recent studies use exposure to light to destroy photoreceptors with one of two paradigms: 1 ) The model developed in David Hyde's laboratory is a chronic light lesion - albino zebrafish are dark-adapted for several days, then exposed to constant, bright illumination ( 3000-20,000 lux) for several days (Thummel et al. 2008a; Vihtelic and Hyde, 2000; Vihtelic et al., 2006). 2) The model developed in our laboratory is an acute light lesion - freeswimming, wild-type pigmented zebrafish are exposed to a fiber optic cable emitting high intensity, wide-spectrum light (>100,000 lux) for 30 min (Bernardos et al., 2007). In the constant light paradigm, rod photoreceptors are more subject to damage than cones, and the dorsal retina is affected, whereas the ventral retina is largely spared (Vihtelic and Hyde, 2000). In the acute light lesion, both cones and rods are destroyed centrally in a wide horizontal zone stretching from nasal to temporal poles of the retina (Qin et al., 2009). In both situations, cone photoreceptors, which are not normally produced in central retina, regenerate, and rod photoreceptors are restored through enhanced proliferative activity in the rod precursor population. Combining an acute, high intensity light lesion followed by chronic bright light exposure in albino zebrafish creates greater loss of cone and rod photoreceptors than either paradigm alone, and triggers an extremely robust proliferative response in the inner retina (Thomas et al., 2012a). A more recent light lesion paradigm applies focused light to a single eye in anesthetized fish, thus allowing the other eye to act as a control but also causing damage to neurons besides the photoreceptors (Weber et al., 2013). Studies of these light lesion paradigms have shown different susceptibility to damage among the photoreceptors as well as different patterns of photoreceptor loss across the whole retina.

Zebrafish genetic models in which photoreceptors degenerate have also been explored for their potential to provide insights into selective regeneration. In the model of chronic rod degeneration in the XOPS:mCFP transgenic line described above (Section 3.2), rod precursors in the ONL proliferate and replace rod photoreceptors (Morris et al., 2008). However, a comparison of two transgenic fish lines in which metranidozole-mediated rod photoreceptor ablation destroys all rod photoreceptors or only a subset of rod 
photoreceptors revealed that Müller glia do contribute to rod photoreceptor regeneration when there is an extensive acute loss of all rod photoreceptors (Montgomery et al., 2010). A mutation in a cone-specific gene in the visual transduction pathway (phosphodiesterase 6) causes cone degeneration, and activation of a regenerative response (Morris et al., 2008). Genetically-targeted, metranidozole-mediated cell ablation of larval zebrafish cones sensitive to ultraviolet light also provokes their regeneration (Fraser et al., 2013).

Recovery of visual function after retinal regeneration in goldfish and zebrafish has been assessed only following ouabainmediated destruction (Kästner and Wolburg, 1982; Lindsey et al., 1995; Mensinger and Powers, 1999; Sherpa et al., 2008; Stenkamp et al., 2001). The electroretinogram (ERG) has been used to evaluate retinal function after regeneration, and visual behaviors tested include the dorsal light reflex (in addition to vestibular cues, fish use the direction of down-welling light from the surface for orientation; when blinded in one eye they roll toward the sighted side), escape response (avoiding a large, dark shape suddenly appearing in the visual field), and optokinetic nystagmus (eye-tracking of moving stimuli). These assays demonstrate that the regenerated retina is functional, but they are not highly quantitative or sophisticated measures of visual performance. Histological examinations of the regenerated retina after ouabain destruction, surgical excision, or acute light lesions reveal obvious defects in patterning of cone photoreceptors. Unlike rods, which all contain the same visual pigment, rhodopsin, most vertebrates have more than one cone opsin gene and therefore different spectral types of cones, each maximally sensitive to different wavelengths of light. In teleost fishes, cones are organized in precise, crystalline mosaic arrays, in which each spectral type of cone has a defined and largely invariant relationship with neighbors of different spectral types (Branchek and Bremiller, 1984; Engström, 1963; Marc and Sperling, 1976). Although all types of cones regenerate, the precise cone mosaic pattern is not restored (Cameron et al., 1997; Cameron and Easter, 1995; Nagashima et al., 2013; Qin et al., 2009; Salbreux et al., 2012; Stenkamp and Cameron, 2002; Stenkamp et al., 2001). How these patterning defects affect visual function is unknown.

The origin of retinal progenitor cells in the regenerating fish retina remained elusive for decades before lineage-tracing studies definitively identified Müller glia as the source of regenerated neurons, as described in the following Section 4.2.1. The original studies of retinal regeneration noted enhanced mitotic activity in the ONL and the CMZ after surgical or neurotoxic lesions (Lombardo, 1968; Maier and Wolburg, 1979), and rod precursors were the obvious candidates - a proliferating pool of retinal progenitors ready-at-hand (Raymond et al., 1988b). However, when photoreceptors in the ONL were selectively destroyed with bright light or laser photocoagulation, PCNA+ or BrdU+ dividing cells with elongated morphology appeared in the INL (Vihtelic and Hyde, 2000; Wu et al., 2001), and Müller glia nuclei translocated apically into the denuded ONL and reentered the cell cycle, as demonstrated by labeling with BrdU (Braisted et al., 1994). Surgical lesions also triggered proliferation of INL cells in the adjacent retinal tissue, which at the time were thought to represent the Pax6+ stem cells at the apex of the rod lineage (Faillace et al., 2002; Otteson et al., 2001), but also included Müller glia (Wu et al., 2001; Yurco and Cameron, 2005).

Thus, the weight of evidence steadily converged toward the conclusion that Müller glia function as radial glial-like stem cells. Müller glia respond to homeostatic signals in the uninjured, growing fish retina that call for more rod photoreceptors, but they are also on alert for injury signals that call for multipotent retinal progenitors to replace other retinal neurons.

\subsection{Injury-induced responses of Müller glia}

\subsubsection{Lineage tracing identifies Müller glia as the source of retinal progenitors}

Three lineage-tracing studies in adult zebrafish with different transgenic reporters together provided evidence that Müller glia are the source of regenerated neurons following retinal injury. Fausett and Goldman (2006) isolated promoter elements from the goldfish $\alpha-1$ tubulin (tuba1a) gene that specifically induce GFP expression in Müller glia and neurogenic clusters after stab wounds to the zebrafish retina. They reported that GFP colocalized with Müller markers GFAP and GS, and that GFP+ Müller glia incorporated BrdU and expressed the retinal progenitor marker Pax6. After 7 days post-lesion (dpl), they found GFP+ cells co-labeled with HuC/D, a marker of retinal ganglion cells and amacrine cells. Subsequent studies employed a Cre-ER/loxP conditional genetic recombination system for permanent fate mapping with double transgenic fish (tuba1a:CreER ${ }^{\mathrm{TM}}$; $\beta$-actin:loxPmCherry-loxP-GFP) (Ramachandran et al., 2010b). The promoter fragment (1016 tubaa1) used to direct expression specifically to proliferating Müller glia after retinal injury is from a gene used as a neuron-specific marker $-\alpha-1$ Tubulin protein is expressed widely in immature neurons in the CNS and is induced in retinal ganglion cells regenerating their axons after optic nerve crush (Hieber et al., 1998; Senut et al., 2004). Therefore, expression of GFP in the neurogenic cluster after retinal stab wounds in the 1016 tuba1a transgenic zebrafish could reflect independent activation of the reporter rather than inheritance from GFP-labeled, proliferating Müller glia.

A second study used the transgenic lines described in Section 3.3.2, in which a glial-specific promoter, zebrafish gfap, directs expression of GFP exclusively to astrocytes in the CNS and Müller glia in the retina (Bernardos and Raymond, 2006). In addition to two transgenic lines with a cytoplasmic GFP reporter (mi2001, mi2002), we also generated another transgenic line with nucleartargeted GFP (Tg[gfap:nGFP]mi2004); in this line, the GFP reporter is strongly expressed in immature Müller glia, down-regulated in fully differentiated cells, but then up-regulated in injury-induced, dedifferentiating Müller glia (Bernardos et al., 2007). The nuclearGFP reporter was especially useful in retinal regeneration studies for unambiguous co-localization with markers of proliferation (e.g., PCNA and BrdU) and transcription factors (e.g., Pax6). After acute light lesions, nGFP+ Müller glia reentered the cell cycle at 1-2 dpl, then the nGFP reporter appeared in Pax6+ multipotent progenitors in the neurogenic clusters in the INL at 3 and $4 \mathrm{dpl}$, in $\mathrm{Crx}+$ photoreceptor progenitors in the ONL at 4 and $5 \mathrm{dpl}$, and finally in immature photoreceptors at 5 and $6 \mathrm{dpl}$, which were identified by morphology and immunostaining with the doublecone-specific antibody, zpr1 (Bernardos et al., 2007). Because neither neuronal progenitors nor photoreceptors express the glial-specific intermediate filament, GFAP, the presence of the GFP reporter in these cells after injury could be attributed to perdurance of the protein inherited from proliferating Müller glia.

A third study (Fimbel et al., 2007) used intraocular injection of ouabain to destroy retinal neurons, primarily in the inner retina, and followed the regenerative response with three different transgenic lines: $\operatorname{Tg}$ (gfap:EGFP)nt, $\mathrm{Tg}$ (olig2:EGFP)vu12, and $\mathrm{Tg}$ (ato$h 7: E G F P) n t$, that express the GFP reporter in Müller glia, retinal progenitors, or differentiating retinal ganglion cells, respectively. They showed that Müller glia (labeled with GFP driven by the glial promoter gfap) reentered the cell cycle within one day, but the level of GFP expression in this transgenic line was not sufficient for lineage tracing. The sequential expression of the three transgenes, driven by the gfap, olig2 (oligodendrocyte transcription factor 2), 
Table 1

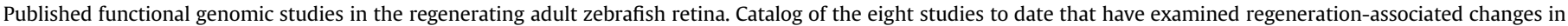

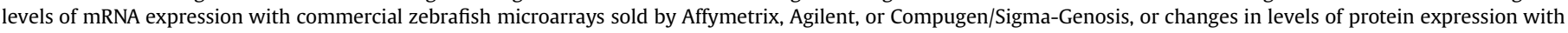
two-dimensional (2D) gel electrophoresis and mass spectrometry (MALDI-TOF).

\begin{tabular}{|c|c|c|c|c|c|c|}
\hline Lesion paradigm & Array & Tissue & Sample times & Dataset & $\begin{array}{l}\text { Genes that } \\
\text { were analyzed }\end{array}$ & Reference \\
\hline Dorsal patch excision ${ }^{a}$ & Microarray (Affymetrix) & Whole retina & $2,3,5,14$ days post-injury & GSE3303 & & Cameron et al., 2005 \\
\hline Constant light lesion $^{\mathrm{b}}$ & $\begin{array}{l}\text { Microarray (Compugen/ } \\
\text { Sigma-Genosis) }\end{array}$ & Whole retina & $\begin{array}{l}16,51,68,96 \mathrm{~h} \text { of light } \\
\text { exposure }\end{array}$ & & olig2, stat3 & $\begin{array}{l}\text { Kassen et al., 2007; } \\
\text { Nelson et al., } 2012\end{array}$ \\
\hline Constant light lesion & Microarray (Affymetrix) & $\begin{array}{l}\text { Laser-capture } \\
\text { microdissected } \\
\text { outer nuclear layer }\end{array}$ & $\begin{array}{l}6,12,24,28 \mathrm{~h} \text { of light } \\
\text { exposure }\end{array}$ & GSE13068 & lgals1l2, pgrna & Craig et al., 2008 \\
\hline Constant light lesion & Microarray (Affymetrix) & Whole retina & $72 \mathrm{~h}$ of light exposure & GSE13999 & $m d k a, m d k b$ & Calinescu et al., 2009 \\
\hline Acute light lesion ${ }^{c}$ & Microarray (Affymetrix) & $\begin{array}{l}\text { FACS sorted Müller } \\
\text { glia and first progenitors } \\
\text { from gfap:gfp }\end{array}$ & $8,16,24,36 \mathrm{~h}$ post lesion & GSE14495 & $\begin{array}{l}\text { mps1, hsp90, } \\
\text { tgif } 1, \operatorname{six} 3 b\end{array}$ & $\begin{array}{l}\text { Lenkowski et al., 2013; } \\
\text { Qin et al., } 2009\end{array}$ \\
\hline $\begin{array}{l}\text { Chronic rod } \\
\text { degeneration }^{\mathrm{d}}\end{array}$ & Microarray (Agilent) & Whole retina & 10-11 month old fish & GSE22221 & $\begin{array}{l}\text { c-myb, insm1a, } \\
\text { sox11b }\end{array}$ & Morris et al., 2011 \\
\hline Stab wound ${ }^{e}$ & Microarray (Agilent) & $\begin{array}{l}\text { FACS sorted Müller } \\
\text { glia-derived } \\
\text { progenitors from } \\
\text { tub1a:GFP relative } \\
\text { to Müller glia in gfap:gfp }\end{array}$ & 4 days post-injury & GFE36191 & insm1a & $\begin{array}{l}\text { Ramachandran } \\
\text { et al., } 2012\end{array}$ \\
\hline Constant light lesion & $\begin{array}{l}\text { 2D protein gel \& } \\
\text { MALDI-TOF }\end{array}$ & Whole retina & $16 \mathrm{~h}$ of light exposure & & $\mathrm{TNF} \alpha$ & Nelson et al., 2013 \\
\hline \multicolumn{7}{|c|}{$\begin{array}{l}\text { a Hitchcock et al., } 1992 . \\
\text { b Vihtelic and Hyde, } 2000 . \\
\text { c } \text { Bernardos et al., } 2007 . \\
\text { d } \text { Morris et al., } 2005 . \\
\text { e Senut et al., 2004. }\end{array}$} \\
\hline
\end{tabular}

then atoh7 promoters, was consistent with the proposal that Müller glia are the source of progenitors that regenerate retinal neurons.

After it was established that Müller glia in the adult zebrafish retina are, in fact, the stem cell source for retinal progenitors that regenerate neurons, Ryan Thummel and colleagues developed a method for targeted knock-down of genes of interest in adult retinas with morpholino oligonucleotides (Thummel et al., 2011, 2008b). They injected intraocularly and then electroporated pcna morpholinos into the adult zebrafish retina immediately prior to initiating a chronic light lesion. Not surprisingly, knock-down of PCNA inhibited photoreceptor regeneration even as late as $28 \mathrm{dpl}$, but unexpectedly, they observed increased cell death in the INL of Müller glia-like cells and a reduction in Müller glia cell numbers (Thummel et al., 2008b). These results suggested that blocking cell division in injury-induced Müller glia promoted their apoptosis, and confirmed that Müller glia are the cellular source of regenerated neurons.

\subsubsection{Müller glia dedifferentiate and express progenitor markers} but they retain a glial identity

A wealth of information has been generated using microarray and proteomics analyses to identify damage signals and the Müller glial response to these signals in zebrafish retina (Table 1) (Calinescu et al., 2009; Cameron et al., 2005; Craig et al., 2008; Kassen et al., 2007; Morris et al., 2011; Nelson et al., 2013; Qin et al., 2009; Ramachandran et al., 2012). These genomic studies have examined post-lesion survival times as early as 6 hours postlesion (hpl) for tissues sampled from the ONL (Craig et al., 2008), $8 \mathrm{hpl}$ for Müller glia with a GFP reporter isolated by fluorescence activated cell sorting (FACS) (Qin et al., 2009), and as late as 14 days post-lesion $(\mathrm{dpl})$ for homogenates of the whole retina (Cameron et al., 2005). Regardless of the lesion paradigm used, the sequence of basic cellular responses to retinal damage in Müller glia are: 1) transient dedifferentiation, 2) asymmetric division, and 3) redifferentiation.

In response to retinal damage, Müller glia transiently increase expression of markers of retinal progenitors and immature Müller glia, indicating that they partially dedifferentiate. After a thermal lesion or acute light lesion, the stem cell marker BLBP is upregulated within one hour, and the multipotent retinal progenitor marker Rx1 is up-regulated within eight hours in Müller glial cells (Nagashima et al., 2013; Raymond et al., 2006). Rx1 protein is also expressed in immature Müller glia near the CMZ and is quickly up-regulated in activated Müller glia after destruction of either photoreceptors or retinal ganglion cells in adult zebrafish (Nagashima et al., 2013). Pax6 expression is used as a marker for multipotent retinal progenitors and is also associated with neurogenic radial glia in the mammalian brain (Hsieh, 2012; Macdonald and Wilson, 1997). Zebrafish have two Pax6 genes pax6a and pax6b; after injury Müller glia up-regulate Pax6 protein (the antibody recognizes both paralogs), and Müller glia-derived progenitors continue to express pax6b and pax6a during regeneration (Bernardos et al., 2007; Raymond et al., 2006; Thummel et al., 2010). Vsx2 (the ortholog of $C h x 10$ ) is another marker of multipotent retinal progenitors in the CMZ that is up-regulated in activated Müller glia (Raymond et al., 2006). Six3 is a critical transcription factor for vertebrate and invertebrate eye development (Loosli et al., 1999; Singh and Tsonis, 2010); it regulates cell proliferation and differentiation by directly interacting with the cell-cycle inhibitor Geminin (Del Bene et al., 2004). Zebrafish have three Six3 paralogs, but only six3b is up-regulated in Müller glia after acute light damage within $8 \mathrm{hpl}$ (Lenkowski et al., 2013; Qin et al., 2009; Sato et al., 2003). Olig2 is a basic helix-loop-helix transcription factor that is important during post-mitotic steps in cell fate determination in selected populations of neurons and glia (Hafler et al., 2012). Based on the expression profile of olig2 in the damaged fish retina, its role in cell fate determination may be conserved during retina regeneration. During a chronic light lesion in an olig2:EGFP transgenic fish line, Müller glia, and potentially the Müller glia-derived progenitors, begin to express the GFP reporter between 51 and $68 \mathrm{~h}$ of light exposure, after Müller glia have divided, and synchronous with increased levels of pax6b transcripts followed by olig2 transcripts in the retina (Thummel et al., 2008b). 
Accompanying the transient increase in retinal progenitor markers in the injury-activated Müller glia, some markers of Müller cell differentiation are reduced. The most dramatic change is in the CRALBP gene paralog that is expressed in Müller glia, rlbp1a, which is down-regulated within four hours after an acute light lesion and disappears by 1 day in Müller glia in the damaged region after either acute light lesion or ouabain injection (Nagashima et al., 2013). Reductions in GS and GFAP have been reported after ouabain injections (Fimbel et al., 2007) or during chronic light lesions (Thummel et al., 2008a), although in other studies, expression of the GFP reporter driven by the gfap promoter and/or immunoreactivity with anti-GFAP is increased in injury-induced Müller glia (Bernardos et al., 2007; Braisted et al., 1994; Lenkowski et al., 2013; Vihtelic et al., 2006; Wu et al., 2001), similar to the gliotic response of mammalian Müller glia (Bringmann et al., 2009; Wohl et al., 2012). Regardless of the levels of expression, both GS and/or GFAP are typically used as specific markers of Müller glia after retinal injuries or cell death (Fausett and Goldman, 2006; Montgomery et al., 2010; Powell et al., 2012; Ramachandran et al., 2011, 2012; Vihtelic and Hyde, 2000; Wan et al., 2012; Yurco and Cameron, 2005).

The continued expression of glial markers by Müller glia in the damaged fish retina indicates that they do not dedifferentiate completely, nor are they reprogrammed to become neuroepithelial cells or retinal progenitor cells, contrary to what is sometimes suggested (Karl and Reh, 2010; Wan et al., 2012). A survey of histology presented in published studies of retinal regeneration indicates that Müller glia retain their apical-basal process throughout the regenerative response; their lateral processes are also present, though remodeled reflecting the loss and subsequent replacement of neurons (Fausett and Goldman, 2006; Gemberling et al., 2013; Gorsuch and Hyde, 2013; Nagashima et al., 2013). In a number of lesion models, including light damage of photoreceptors and destruction of inner retinal neurons with ouabain, the overall lamination of the retina remains intact, and Müller glia continue to provide structural support; in fact, without Müller glia, the cytoarchitecture of the residual differentiated retina could not be maintained. For example, when Müller glia are ablated with a glial toxin, the laminar structure of the retina is severely disrupted (Rich et al., 1995), and genetically targeted conditional ablation of Müller glia in mice leads to photoreceptor apoptosis, breakdown of the blood-retinal barrier, and intraretinal neovascularlization (Shen et al., 2012). Conditional knock-out of Sox2 in postnatal murine Müller glia forces their reentry into the cell cycle, withdrawal of their basal processes, and symmetric division resulting in loss of Müller glia and severe laminar defects, especially in the outer retina (Surzenko et al., 2013). Together these data suggest that successful regeneration of retinal neurons in situ requires the continued homeostatic structural and metabolic support functions of Müller glia, as well as a source of retinal progenitors that differentiate into neurons.

\subsubsection{Regeneration 'niches' - neurogenic clusters derive from a} self-renewing mitotic division of a Müller glia

In all of the studies reviewed above, no matter the type of retinal damage or species of teleost fish, investigators have described several distinct types of proliferating cells within the retina (Fig. 2) including: 1 ) reactive microglia/macrophages (identified with the specific antibody, 4C4), which migrate into the lesion to phagocytize cellular debris; 2 ) elongated clusters of proliferating progenitor cells closely associated with radial processes of Müller glia in the INL; and 3) scattered rod precursors in the ONL. We have previously described the neurogenic clusters in the INL as regeneration 'niches', analogous to the retinal stem cell niche in the CMZ (Raymond et al., 2006), a concept that has been helpful to understanding the behavior of somatic stem cells elsewhere in the body (Wagers, 2012). What is somewhat unique about the retinal regeneration niche is that Müller glia function as both stem cells and niche cells because they produce and then support the neurogenic cluster of retinal progenitors that differentiate into neurons.

The gfap:nGFP transgenic zebrafish line that expresses nuclear GFP in immature and injury-induced Müller glia in the retina (Bernardos et al., 2007), and which facilitated lineage tracing studies described above (Section 4.2.1), also confirmed previous observations in goldfish of IKNM by Müller glia nuclei as early as 1 day after photoreceptor damage (Braisted et al., 1994). After photoreceptor destruction, mitosis occurs at the apical surface (OLM); after inner retinal neuron destruction, Müller nuclei translocate apically but do not penetrate through the intact layer of photoreceptors (ONL), and mitosis occurs at inner border of the ONL (Nagashima et al., 2013). This initial mitotic division of the Müller glia occurs between 1 and 2 dpl after photoreceptor damage and is delayed by approximately one day after loss of inner retinal lesions (Bernardos et al., 2007; Nagashima et al., 2013; Thummel et al., 2008a).

Within a few days after injury, neurogenic clusters of rapidly dividing retinal progenitors are present in the INL. A question that has not been directly addressed until recently is whether Müller glia divide repeatedly to generate neuronal progenitors or whether the glial-derived progenitors divide symmetrically to generate neurogenic clusters surrounding each Müller glia, similar to transitamplifying progenitors in the vertebrate brain. Thummel et al. (2010) suggested that multiple divisions of Müller glia are not required to generate neurogenic clusters, since morpholinomediated knock-down of Pax6b did not affect the initial Müller glial division, but did inhibit the early divisions of the retinal progenitors, resulting in the absence of neurogenic clusters and subsequent failure of cone regeneration after a chronic light lesion. To examine this question directly, we used a sequential labeling scheme with two thymidine analogs: BrdU and EdU (Nagashima et al., 2013). We exposed adult zebrafish with acute light lesions to EdU (delivered continuously in the aquarium water) from 20 to $36 \mathrm{hpl}$, which corresponds to the S-phase of the initial cell cycle in Müller glia. After a 6-h chase period, we then exposed fish to continuous BrdU from 42 to $72 \mathrm{hpl}$. If Müller glia divided only once, they would incorporate EdU but not BrdU; if they underwent multiple rounds of division, they would incorporate both EdU and BrdU. We found that $86 \%$ of the EdU-labeled cells were BrdUnegative but surrounded by a cluster of BrdU+ cells, and neurogenic clusters of weakly EdU+ progenitors (labeled with the inducible nGFP transgenic reporter) were typically associated with a single strongly EdU + cell, consistent with dilution of the EdU label by subsequent divisions of the glia-derived retinal progenitors. Additional evidence from light damaged retinas that Müller glia undergo one asymmetric, self-renewing division includes the following: 1) in the gfap:GFP transgenic line, neurogenic clusters at $3 \mathrm{dpl}$ contain weakly GFP+ mitotic (PCNA+) elongated (migratory) retinal progenitors associated with a single, strongly GFP+, non-mitotic (PCNA-) cell with distinctive Müller glial morphology, including a polygonal cell body/nucleus and a GFAP+ radial process that expresses the immunoglobin adhesion molecule, Alcama (see Section 5.7 below), and 2) the number of Müller glia in the lesioned region remains constant throughout the regeneration process (Nagashima et al., 2013). Therefore, similar to mitotic divisions of radial glia in the vertebrate brain (Gotz and Huttner, 2005), IKNM in Müller glia results in an asymmetric, self-renewing division.

Although PCNA expression has often been used to monitor cell proliferation during the regenerative response, and the PCNA 
morpholino studies cited in Section 4.2.1 demonstrated that proliferation of Müller glia is necessary for retinal neuron regeneration, other cell-cycle regulators are also critical for a successful regenerative response. For example, zebrafish with a temperaturesensitive mutation in the mitotic checkpoint protein, mps1 (multipolar spindle1), when maintained at the restrictive temperature after acute light lesion, fail to regenerate cone photoreceptors (Qin et al., 2009). Expression of mps1 increases when Müller glia begin to divide between 24 and $36 \mathrm{hpl}$ and remains elevated at least through $48 \mathrm{hpl}$ (Qin et al., 2009), when their progeny constitute a rapidly amplifying population of retinal progenitor cells.

In the developing mouse retina, the cyclin-dependent kinase inhibitor $p 27^{k i p 1}$ is up-regulated in retinal progenitors at withdrawal from the cell cycle (Levine et al., 2000). In zebrafish Müller glia, $p 27^{\text {kip } 1}$ is down-regulated by $8 \mathrm{~h}$ after acute light lesion (Qin et al., 2009) and by $16 \mathrm{~h}$ of light exposure in a chronic lesion (Kassen et al., 2007). Similarly, mouse Müller glia down-regulate p27 $7^{k i p 1}$ and up-regulate GFAP after injury (Dyer and Cepko, 2000a), and in both the injured and uninjured mouse retina, $p 27^{k i p 1}$ deficiency leads to reactive gliosis in Müller glia (Dyer and Cepko, 2000a; Vazquez-Chona et al., 2011). During mouse retinal development, $p 57^{k i p 2}$ is expressed in the final cell cycle and in a subset of progenitors different from the $p 27^{\text {kip } 1}$-positive cells (Dyer and Cepko, 2000b, 2001). In zebrafish whole retina lysates, $p 57^{k i p 2}$ is up-regulated by $16 \mathrm{~h}$ after the initiation of a chronic light lesion (Kassen et al., 2007) and $8 \mathrm{~h}$ after stab wounds (Ramachandran et al., 2012), indicating tight control of the cell cycle early in regeneration when Müller glia are dividing (Kassen et al., 2007). Because rod precursors begin proliferating by $16 \mathrm{~h}$ in a chronic light lesion (Thummel et al., 2008a), expression of these two cell cycle inhibitors may control cell cycle dynamics in both Müller glia and rod precursors during the regenerative response.

In summary, Müller glia transiently and partially dedifferentiate and re-express some progenitor markers. However, Müller glia themselves do not become retinal progenitor cells. Rather, similar to neural stem cells in the adult mammalian brain, Müller glia divide asymmetrically to generate a rapidly proliferating retinal progenitor cell that builds the neurogenic cluster. The Müller glial cell and the glial-derived neuronal progenitors are tightly associated in a 'regeneration niche', that likely allows for specific signaling interactions that support neurogenesis within the surrounding differentiated and functioning retinal tissue, similar to the neural stem cell niche in the adult mammalian brain (Fuentealba et al., 2012; Morrens et al., 2012).

\section{Complex network of signaling pathways necessary for regeneration}

The flowchart in Fig. 4 provides a summary overview of the signaling pathways discussed in this section.

\subsection{Stress response (HSP60, Stat3)}

A classic stress response to retinal damage occurs in both the damaged and dying neurons and the Müller glia in adult fish retinas. Apoptotic photoreceptor nuclei can be labeled by TUNEL 6$12 \mathrm{~h}$ after damage in light lesion paradigms, peaking by $24 \mathrm{~h}$ after light lesion in both acute and chronic lesions (Bernardos et al., 2007; Craig et al., 2008; Vihtelic and Hyde, 2000), but this time course is slower than other transcriptional changes that can be detected in the damaged ONL and reactive Müller glia (Craig et al., 2008; Qin et al., 2009). Broadly speaking, after retinal damage, several stress-response transcripts and proteins are rapidly induced (as measured by microarray analysis of retinal homogenates) including several heat shock proteins and proteins that protect

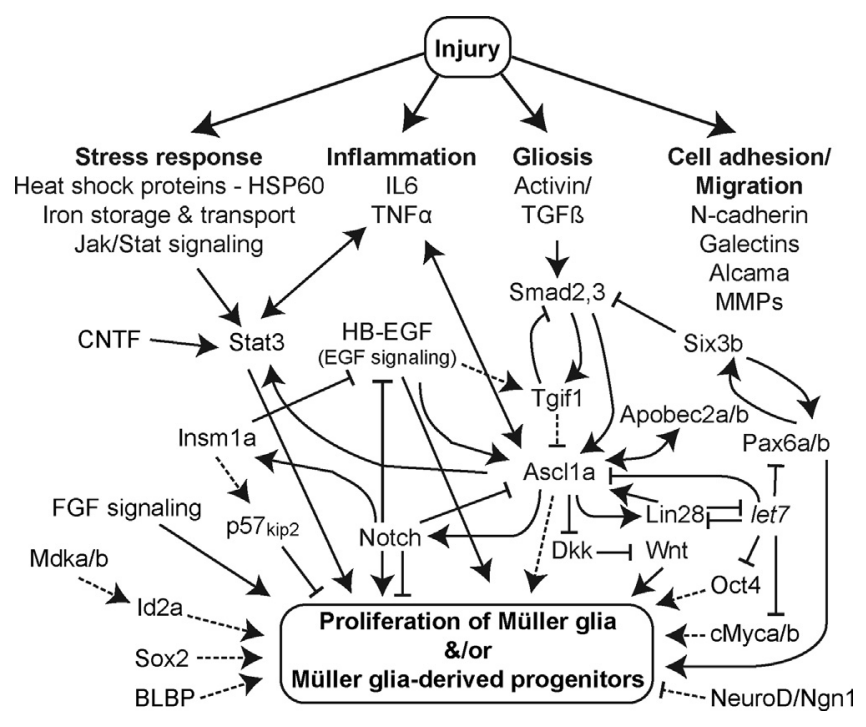

Fig. 4. Overview of signaling pathways that regulate proliferation of Müller glia and Müller glia-derived progenitors during retinal regeneration in adult zebrafish. Following retinal injury, expression of factors associated with a stress response, inflammation, gliosis, and cell adhesion and migration are modified to regulate cell proliferation leading to regeneration of retinal neurons. Solid lines indicate regulatory interactions that have been described in the literature; dashed lines indicate suggested or indirect interactions. See Section 5 for references and further discussion of when and where these various factors are expressed during retinal regeneration.

against oxidative stress; these are likely indicative of the damage response in photoreceptors after the initiation of a chronic light lesion (Craig et al., 2008; Kassen et al., 2007; Nelson et al., 2013). Müller glia also initiate a stress response after neuronal damage: hspd1 transcription increases within $8 \mathrm{hpl}$ and remains elevated at least through $48 \mathrm{hpl}$, along with at least 10 other heat-shock proteins and factors (Qin et al., 2009). The hspd1 gene encodes heat shock 60-kDa protein 1 (HSP60), a mitochondrial chaperone previously shown to be necessary for fin regeneration (Makino et al., 2005). Loss of hspd1 function in temperature-sensitive mutant zebrafish significantly reduces proliferation after acute light lesion in Müller glia that express the retinal progenitor gene pax6 (Qin et al., 2009; Section 5.6). It is likely that heat shock proteins in general, and hspd1 specifically, are critical protein chaperones throughout the regenerative response: hspd1 mutants have defects in cone photoreceptor regeneration, and hspd1 expression is maintained in the Müller glia after they have divided for at least $48 \mathrm{~h}$ after light damage (Qin et al., 2009), and in whole retinal homogenates through at least 7 days after stab wounds (Ramachandran et al., 2010a).

Microarray analysis of alterations in levels of gene transcripts indicates dramatic metabolic changes following retinal damage. For example, regulation of iron, while it has not been studied in the context of retinal neuron regeneration in zebrafish, is important for visual function and health of the retina (Burke and Smith, 1981; Gnana-Prakasam et al., 2010; Picard et al., 2011). Excess iron is highly toxic to cells because it easily generates reactive oxygen species, and retinas of mice with reduced ferritin protein (used for iron storage) lose significantly more photoreceptors in response to light damage (Picard et al., 2011). Within 16 h after initiation of light damage in adult zebrafish retinas, proteins associated with iron storage and transport are significantly up-regulated, including transferrin (iron transporter), hemopexin (binds heme), and ceruloplasmin (oxidizes iron) (Nelson et al., 2013). In contrast, following intense light lesion, Müller glia down-regulate transcripts of transferrin, iron-regulated ion transporter, slc40a1 (iron exporter), 
and hypoxia-induced factor 1a (regulates hypoxia-responsive genes), whereas ferritin (iron storage) and many cytochrome p450 enzymes that use iron are up-regulated (Qin et al., 2009). Together these data suggest that light damage-induced hypoxia necessitates a rapid response to prevent further damage from free radicals generated by iron. In Müller glia the overall metabolic response likely functions to bind and store iron as part of a neuroprotective response to a lesion, while in the retina as a whole, iron oxidation and transport are increased.

Widely known to be critical for development and function of the immune system, the Jak-Stat (Janus kinase/Signal transducer and activator of transcription) signaling pathway is also important for glial development (Bonni et al., 1997), the stress response to neuronal damage (Zhang et al., 2005), and maintenance of selfrenewal properties of ESCs (Niwa et al., 1998). In response to retinal damage in adult zebrafish, stat3 expression increases quickly in the dorsal retina by $5 \mathrm{~h}$ after light-onset in a chronic light lesion (Nelson et al., 2012) and specifically in Müller glia by $8 \mathrm{~h}$ after an acute light lesion (Qin et al., 2009). Phosphorylated Stat3 levels also increase during the first $68 \mathrm{~h}$ of light damage (Kassen et al., 2007). The roles of Stat3 in retinal regeneration are likely complex, as is true for other signaling pathways discussed in this review. Stat3 is not required for the neuroprotective effects of CNTF in chronic light damage (Kassen et al., 2009), so it is perhaps not surprising that morpholino-mediated knock-down of Stat3 does not protect zebrafish photoreceptors from light-induced cell death (Nelson et al., 2012). In contrast, mouse retinal ganglion cells transfected with a constitutively active Stat 3 can better survive various insults both in vitro and in vivo (Zhang et al., 2008).

Interestingly, Stat3 levels in Müller glia increase in response to light damage in both adult zebrafish and adult mice, yet mouse Müller glia do not typically divide in this situation (Joly et al., 2011), nor do they initiate a program of photoreceptor regeneration. Only a subset of zebrafish Müller glia that express Stat3 also express PCNA after photoreceptor damage (Nelson et al., 2012), and a subset of embryonic mouse retinal progenitors that are phospho-Stat3positive also incorporate BrdU in response to CNTF treatment (Zhang et al., 2005). Therefore, while Stat3 expression is required for a maximal proliferative response from the population of retinal Müller glia after photoreceptor or inner neuron destruction in adult zebrafish, increased Stat 3 expression in response to damage is not sufficient to activate the regeneration program in the mouse retina.

\subsection{Secreted growth factors and cytokines (FGF, HB-EGF, TNFa, TGF $\beta /$ Tgif1, CNTF, Mdka/b)}

In addition to determining the transcriptional changes that occur in Müller glia as an early response to retinal damage, a key goal of many studies has been to determine what secreted factors signal retinal damage and/or cell loss, and how that signal is transduced in the Müller glia. Signals that are critical for successful retinal neuron regeneration are likely to be detected in damaged and dying neurons, reactive microglia, as well as Müller glia. Particularly with light-lesion paradigms, in which photoreceptors are the only damaged neurons, several research groups have identified factors secreted by dying photoreceptors that likely have a role in initiating the regenerative response of Müller glia. Secreted factors present in the ONL after acute light lesion that may be important for signaling to the Müller glia after injury include those that decrease after lesion (follistatin-a, nerve growth factor- $\beta$, progranulin- $a$, and insulin- $\beta$ ) and others that increase (galectins, midkine-related growth factors, activin, and connective tissue growth factor) (Craig et al., 2008). Noteworthy changes also occur in the expression of many signaling pathways downstream of these secreted factors. Future studies are needed to determine how adult zebrafish Müller glia respond to these signals and to compare their responses to those of adult mammalian Müller glia that do not exhibit robust regeneration.

Microglia, the resident macrophages of the retina, become reactive within $8 \mathrm{~h}$ after acute light lesion or ouabain injection and migrate into the damaged areas where they phagocytize cell debris (Fig. 2) (Bernardos et al., 2007; Craig et al., 2010; Nagashima et al., 2013; Nelson et al., 2013). Because microglia also release cytokines, they may contribute to the initial damage response. Two ligands in the TGF $\beta$ signaling pathway inhibit the activation of mammalian microglia in vitro (TGF $\beta$ ligand, Suzumura et al., 1993) and in vivo, where they also regulate the inflammatory response to neuron excitotoxicity (activin A, Abdipranoto-Cowley et al., 2009). In the zebrafish brain, traumatic injury induces inflammation and a regenerative response, whereas immunosuppression impairs the regenerative response, and induction of inflammation, even without injury, induces a proliferative neurogenic reaction (Kyritsis et al., 2012). As with zebrafish brain regeneration, retinal regeneration may depend on an inflammatory response initiated by activated microglia, a topic that deserves further investigation.

Signaling by the inflammatory cytokine TNF (Tumor Necrosis Factor) has been implicated in proliferative gliosis in the mammalian retina (Bringmann et al., 2009), and TNF $\alpha$ is required for proliferation of Müller glia during retinal neuron regeneration in zebrafish. In whole retina analyses, tnf receptor-21 transcripts (Kassen et al., 2007) and TNF receptor-associated protein-1 (Nelson et al., 2013) both increase by $16 \mathrm{~h}$ after initiation of a chronic light lesion. Further studies showed that the TNF $\alpha$ ligand is expressed in dying photoreceptors and inner retinal neurons after light lesion, and that Stat3 regulates subsequent TNF $\alpha$ expression in Müller glia (Nelson et al., 2013). It is apparent that TNF $\alpha$ is a critical signal for regeneration after neuron damage, as morpholino-mediated knock-down of TNF $\alpha$ in either the dying neurons (early) or the Müller glia (later) significantly reduces proliferation and expression of stat3 and ascl1a (Nelson et al., 2013). David Hyde and colleagues suggest that inhibiting TNF $\alpha$ delays regeneration because after $24 \mathrm{~h}$ of recovery from a chronic lesion (104 h after morpholino electroporation), TNF $\alpha$ morphant retinas have significantly more PCNA-positive nuclei than controls (Nelson et al., 2013). It will be important to establish if elevated TNF $\alpha$ expression is sustained throughout the regenerative process in the retina; alternatively, when the TNF $\alpha$ morpholinos lose effectiveness, the morphant phenotype may be rescued, raising the possibility that delayed TNF $\alpha$ treatment could rescue other regeneration defects.

Both retinal progenitor cells and Müller glia proliferate in vitro and in vivo in response to several growth factors, including EGF (Epidermal Growth Factor), FGF (Fibroblast Growth Factor), and PDGF (Platelet-Derived Growth Factor) (Close et al., 2006; Fischer et al., 2002; Ikeda and Puro, 1994, 1995; Karl et al., 2008; Lillien and Cepko, 1992; Negishi and Shinagawa, 1993; Puro, 1995). In the early 1990's, researchers suggested that this proliferative response to growth factors may be important for the ability of fish to regenerate retinal neurons (Hitchcock and Raymond, 1992; Negishi and Shinagawa, 1993). In fact, after neuronal damage and intraocular injection of EGF, FGF1, or FGF1 and insulin, mouse Müller glia re-express progenitor markers, proliferate, and generate a few cells that express specific markers of retinal neurons (Karl et al., 2008). To date, the growth factors that have been studied in the context of retinal neuron regeneration in zebrafish have a variety of roles that may vary between retinal lesion paradigms (Nelson et al., 2013; Wan et al., 2012).

Signaling through EGF receptors (EGFR) can be initiated by secreted EGF or heparin-binding EGF (HB-EGF), and both are mitogenic for Müller glia. Studies exploring the downstream signaling of EGFR in adult zebrafish retinal regeneration underscore 
the complexity of mounting a successful regenerative response. After stab lesions in adult zebrafish, $h b$-egfa is up-regulated within one hour in the whole retina and is expressed in Müller glia after injury; morpholino-mediated knock-down of $h b$-egfa reduces the number of proliferating progenitor cells through 4 dpi (Wan et al., 2012). Similarly, inhibitors of EGFR and MAPK/ERK also reduce the number of proliferating progenitors after stab wounds (Wan et al., 2012). Expression of $h b$-egfa also increases in light-damaged retinas, but $h b$-egfa morpholino-mediated knock-down does not reduce proliferation in light-damaged retinas (Nelson et al., 2013), suggesting that HB-EGF regulation of Müller glial proliferation may be context-dependent. Studies of Müller glial response to exogenous HB-EGF in the undamaged retina have also reported conflicting results (Nelson et al., 2013; Wan et al., 2012).

Expression of $h b$-egf is negatively regulated by the transcriptional repressor insm1a, which is up-regulated pan-retinally within $6 \mathrm{~h}$ after stab wound lesions; morpholino-mediated knock-down of Insm1a at $4 \mathrm{dpl}$ increases expression of $h b$-egfa (Ramachandran et al., 2012). Additionally, chromatin immunoprecipitation analysis showed that endogenous Insm $1 \mathrm{a}$ in the injured retina binds the promoter of $h b$-egf, suggesting a direct repression of transcription. Gorsuch and Hyde (2013) suggest that Insm1a repression of HBEGF may maintain quiescence in Müller glia that surround a lesion site; similar effects of HB-EGF in defining the zone of responsive Müller glia may be mediated through Wnt $/ \beta$-catenin and Notch signaling, as discussed below (Section 5.7).

Mammalian Müller glia exhibit changes in EGFR signaling that parallel those in the adult zebrafish retina. Human Müller glia express HB-EGF in proliferative vitreoretinopathy (PVR) - a disease in which Müller glia proliferate and contribute to a glial scar anterior to the retina (Hollborn et al., 2005). Mammalian Müller glia in vitro proliferate via ERK1/2 (Extracellular Receptor Kinase) signaling and migrate in response to HB-EGF (Hollborn et al., 2005); after light damage in vivo, Müller glia increase expression of EGFR, incorporate BrdU, and generate proliferating cells located in the ONL (Close et al., 2006). In perinatal rat retinas, EGF signaling increases Müller glia proliferation through activation of both ERK/MAPK (MitogenActivated Protein Kinase) and PI3/AKT (Phosphoinositide 3-kinase/ serine-threonine kinase Akt, cellular homolog of viral oncogene, $\mathrm{v}$ Akt) signaling; the latter is also critical for mediating proliferation via BMP signaling (Ueki and Reh, 2013). Signaling via the PI3/AKT pathway has not yet been investigated in retinal regeneration in fishes.

FGF signaling is important for both maintenance of mature photoreceptors and photoreceptor regeneration (Hochmann et al., 2012; Qin et al., 2011). Two studies have used a heat-shock inducible dominant-negative fgfr1a transgenic fish line ( $h s p 70: d n-$ fgfr1a) that allows for temporal, but not spatial, control of transgene expression to manipulate FGF signaling during photoreceptor regeneration (Hochmann et al., 2012; Qin et al., 2011). In the absence of injury, expression of $d n$-fgfr1 leads to degeneration of photoreceptors (Hochmann et al., 2012; Qin et al., 2011). Following acute light lesion, inhibition of FGF signaling with the dn-fgfr1a does not affect the initial response of Müller glia to light lesions (Qin et al., 2011), but does reduce proliferation of neurogenic progenitors when the transgenic fish carry two copies of the transgene (Hochmann et al., 2012). When fish carry only one copy of $d n$-fgfr1, rod, but not cone, photoreceptor regeneration is impaired, and intravitreal injection of FGF2 induces proliferation of rod progenitors in the ONL (Qin et al., 2011). Taken together these two studies indicate that the levels of FGF signaling are critical for regulating the regenerative response.

TGF $\beta$ (Transforming Growth Factor $\beta$ ) signaling can antagonize the proliferative response to growth factors in Müller glia (Close et al., 2005; Ikeda and Puro, 1994, 1995; Lenkowski et al., 2013;
Rohrer and Stell, 1994; Wachs et al., 2006). Mammalian glia proliferate to generate glial scars in response to increased canonical TGF $\beta$ signaling, which is mediated by phospho-Smad2 and phospho-Smad3. Regulation of canonical TGF $\beta$ signaling is exquisitely context-dependent and time-dependent in the regenerating adult zebrafish retina. After acute light lesions, the initial phase includes rapid up-regulation of the TGF $\beta$-family ligand, activin, in the ONL by $6 \mathrm{hpl}$ (Craig et al., 2008), while targets of TGF $\beta$ signaling - tgf $\beta i$ (transforming growth factor, beta- induced), tgif1 (tgf $\beta$ induced factor homeobox 1), and ascl1 a - are up-regulated in Müller glia by 8 hpl (Lenkowski et al., 2013; Qin et al., 2009). Follistatin, which binds to and neutralizes activin, is up-regulated at $12 \mathrm{hpl}$ in the ONL (Craig et al., 2008) and at $16 \mathrm{hpl}$ in the whole retina, but is down-regulated in Müller glia at least through $36 \mathrm{hpl}$ (Qin et al., 2009). These data suggest that dying photoreceptors (or reactive microglia) in the ONL secrete follistatin in response to light damage, possibly as part of a feedback loop to antagonize the activin that is released after injury.

Transcriptional profiling of Müller glia after acute light lesion suggests that canonical TGF $\beta$ signaling is initially up-regulated, then quickly down-regulated by $16 \mathrm{hpl}$; the extracellular matrix protein $\operatorname{tg} f \beta i$ is initially up-regulated, but then rapidly down-regulated, while levels of the TGF $\beta$ transcriptional corepressors tgif1 and $s i x 3 b$ remain elevated and activin receptors depressed through at least $36 \mathrm{hpl}$ (Qin et al., 2009). The proliferative response to an acute light lesion is significantly dampened in mutant zebrafish expressing a truncated Tgif1 protein or following morpholino-mediated knock-down of Six3a/b (Lenkowski et al., 2013). Furthermore, significantly fewer cone photoreceptors are regenerated in fish expressing the truncated Tgif1 or in fish expressing only truncated forms of Tgif1 and Six3b; retinas in these fish have higher levels of expression of TGF $\beta$ signaling targets, including ascl1a, indicating that TGF $\beta$ signaling must be repressed for successful retina regeneration and that ascl1a over-expression is not sufficient to compensate for misregulation of TGF $\beta$ signaling (Lenkowski et al., 2013). TGF $\beta$ receptors physically interact with tight junction proteins, and non-canonical TGF $\beta$ signaling can dissociate cell-cell junctions, facilitating epithelial-mesenchymal transition (EMT) and migratory behavior, without affecting canonical TGF $\beta$ signaling (Barrios-Rodiles et al., 2005). Since EMT and cell migration are associated with the mammalian glial scarring response, elucidating a mechanism by which canonical TGF $\beta$ signaling could be inhibited, while allowing for dedifferentiation and non-gliotic proliferation that occurs in adult zebrafish, possibly through corepressors such as Tgif1 and Six3, would be attractive for translational mammalian studies and potential therapeutic treatments for retinal degeneration. Interestingly, decorin, a naturally occurring TGF $\beta$ inhibitor, is already used to treat surgical glaucoma patients, and a recent study of rabbit PVR shows that decorin treatment prior to surgery reduces the scarring response (Nassar et al., 2011).

Several additional secreted factors are expressed after retinal damage and may have important roles in retinal neuron regeneration. For example, two zebrafish homologs of the heparin-binding growth factor Midkine, midkine $a(m d k a)$ and midkine $b$, may be important for regulating cell cycle dynamics during retinal regeneration. During retinal development, mdka positively regulates the transcriptional repressor id2a (inhibitor of DNA-binding 2a), which modulates cell cycle progression from S- to M-phase without altering cell cycle timing or exit (Luo et al., 2012), and both midkines are re-expressed in proliferating Müller glia and photoreceptor progenitors after acute light lesion (Calinescu et al., 2009). The growth factor CNTF has dual roles in regeneration. In a chronic light lesion paradigm, exogenous CNTF injections are neuroprotective via MAPK signaling, and as a result Müller glia exhibit minimal proliferative response to light exposure, whereas CNTF 
injections in unlesioned retinas can induce Müller glial proliferation via a Stat3 mechanism (Kassen et al., 2009). In the mammalian CNS, CNTF induces GFAP expression and glial fate via Stat3 signaling (Bonni et al., 1997; Wang et al., 2002b), and in mammalian Müller glia, exposure to CNTF induces genes associated with inflammation and gliosis (Xue et al., 2011). This dichotomy in response to CNTF may reflect a divergence in the responses of Müller glia in mammals and zebrafish to retinal damage.

As discussed in Section 3.2, the transcriptional repressor insm1a has an integral role in regulating cell cycle exit and differentiation of retinal progenitors, especially rod photoreceptors, during zebrafish development (Forbes-Osborne et al., 2013). A parallel role during retinal regeneration has also been described - insm1a indirectly regulates $p 57^{k i p 2}$ via repression of a repressor of $p 57^{k i p 2}$, $b c 111 a$ - and at 4 days after stab wound, co-expression of insm1a and $p 57^{k i p 2}$ in Müller glia-derived retinal progenitors drives cell cycle exit (Ramachandran et al., 2012). Morpholino-mediated knock-down of Insm1 at $4 \mathrm{dpl}$ expands the proliferative zone around the injury, but after 30 days, BrdU-labeled Müller glia survive in the expanded proliferative zone and regenerated neurons do not, for reasons that are not known (Ramachandran et al., 2012).

\subsection{Retinal determination transcription factors (Six3, Pax6)}

Several retinal determination factors are re-expressed in activated Müller glia in the first $24 \mathrm{~h}$ after retinal damage, including six3, pax6, rx1, olig2, and vsx2, as discussed in Section 4.2.2. These studies suggest that in response to retinal damage Müller glia partially dedifferentiate, as reflected by increased expression of markers of retinal progenitors and of immature Müller glia.

Of the retinal progenitor markers mentioned in Section 4.2.2, we will continue the discussion of six 3 and $p a x 6 a / b$, as these two in particular have been experimentally manipulated in order to characterize their importance in retinal regeneration. Although six $3 b$ is the only zebrafish Six3 paralog that is up-regulated in Müller glia after acute light lesion, a six3b null mutant does not exhibit an apparent regeneration defect (Lenkowski et al., 2013). However, morpholino-mediated knock-down of Six3 (both Six3a and Six $3 b$ are targeted by the morpholino) causes a significant reduction in retinal progenitor proliferation during a chronic light lesion, indicating a potential redundancy between Six3a and Six3b in the regenerative response that is not apparent in wild-type, lesioned fish (Lenkowski et al., 2013). Six3 can also inhibit nodal/ TGF $\beta$ signaling during zebrafish gastrulation (Inbal et al., 2007), and when six $3 b$ and the TGF $\beta$ corepressor tgif1 are both mutated, the retinal regeneration defect is enhanced (Lenkowski et al., 2013). Six3 also demonstrates transcriptional cross-regulation with other key retinal determination factors that likely play a role in regeneration, such as Pax6 (Carl et al., 2002; Loosli et al., 1998; Wargelius et al., 2003).

Expression of Pax6 is critical for many aspects of retinal development, from patterning the early eye field, to regulating the cell cycle and retinal neuron differentiation (as reviewed in Shaham et al., 2012). Pax6 expression in adult zebrafish Müller glia, albeit at low levels, has been associated with their capacity to function as retinal stem cells. Surprisingly, a single cell microarray analysis showed that Pax6 is also expressed at low levels in murine Müller glia (Roesch et al., 2008), and furthermore, murine Müller glia upregulate Pax6 in response to retinal neuron damage, even when they do not proliferate (Joly et al., 2011). Zebrafish have two Pax6 paralogs that differ only slightly in their N-terminal region. This small sequence difference allowed Ryan Thummel and colleagues to specifically target pax6a and pax6b with morpholinos immediately prior to the onset of a chronic light lesion (Thummel et al., 2010). Neither Pax6a nor Pax6b was required for the initial cell division of injury-induced Müller glia, however, knock-down of Pax6b blocked the initial division of retinal progenitors, and knockdown of Pax6a blocked the later amplifying divisions of progenitors (Thummel et al., 2010). Interestingly, pax6a/pax6b double morphant retinas had more proliferation in the ONL, where the endogenous rod precursors reside, and also showed successful regeneration of rods at $28 \mathrm{dpl}$, but not cones (Thummel et al., 2010). This indicates that Pax6a/6b are required for cone photoreceptor regeneration but are not necessary for enhanced production of rod photoreceptors mediated by rod precursors (Thummel et al., 2010). These observations also support the idea that rod precursors are committed to the rod lineage and are unable to dedifferentiate to participate directly in cone photoreceptor regeneration.

How pax $6 a / b$ are regulated during retinal regeneration is still unclear: in vitro studies suggest that pax6b is downstream of an ascl1a-let7 microRNA regulatory network (Ramachandran et al., 2010a), which is discussed below (Section 5.4). Although ascl1a and the cytidine deaminases apobec $2 a / 2 b$ (apolipoprotein B mRNA editing enzyme, catalytic polypeptide-like $2 \mathrm{a} / 2 \mathrm{~b}$, which are involved in DNA methylation) positively regulate each other in Müller glial-derived progenitors, expression of $p a x 6 a / b$ is not altered by morpholino-mediated knock-down of apobec $2 a / b$ (Powell et al., 2012). Additional in vivo data provide evidence for regulation of pax6a/b by Wnt signaling (Ramachandran et al., 2011), HB-EGF signaling (Wan et al., 2012), and possibly ascl1a (Fausett et al., 2008; Ramachandran et al., 2011).

\subsection{Proneural genes and miRNA regulation (Acsl1a, Lin28/Let7, NeuroD, Ngn1)}

The proneural basic helix-loop-helix transcription factor ascl1a occupies a critical position at the interface of several canonical signaling pathways that are activated during retinal regeneration, including Wnt and Notch signaling, as discussed in the next section. Initial studies showed that ascl1a is up-regulated in Müller glia within $6 \mathrm{~h}$ after stab wounds, and its expression is critical for proliferation after injury (Fausett et al., 2008). Morpholinomediated knock-down of the transcriptional repressor Insm1a at the time of injury leads to small but significant increases in ascl1a (Ramachandran et al., 2012). In Müller glia and retinal progenitors, ascl1a levels continue to rise after acute light damage until at least $36 \mathrm{hpl}$ (Qin et al., 2009), and expression levels remain elevated as late as 7 days after injury (Cameron et al., 2005). While morpolino knock-down of Ascl1a inhibits proliferation after retinal injury (Fausett et al., 2008), expression of ascl1a is not sufficient for successful regeneration when canonical TGF $\beta$ signaling is misregulated (Lenkowski et al., 2013) or with reduced expression of insm1a (Ramachandran et al., 2012).

Chromatin immunoprecipitation studies in vitro by Daniel Goldman and colleagues showed that Ascl1a directly binds to the promoter of the pluripotency factor and RNA-binding protein lin28 and thus could indirectly regulate the microRNA let-7 via transcription of the lin28 repressor (Ramachandran et al., 2010a). Regulation between Ascl1a and Lin28 in vivo still requires further clarification: morpholino-mediated knock-down of Lin28 in stab wound lesions had no effect on ascl1a transcript levels (Ramachandran et al., 2010a), but morpholino-mediated knockdown of Lin28 during photoreceptor regeneration abolished Ascl1a protein (Nelson et al., 2012). A recent study of murine retinal development indicates that microRNAs let-7, mi-9, and mi-125 can maintain an "early competence state" in retinal progenitor cells, and experimental manipulation of their expression alters the distribution of neuron subtypes (La Torre et al., 2013); further studies of microRNA expression in retinal regeneration may help resolve contradictory studies. In transfected HEK cells, let-7 can suppress 
Ascl1a, Hspd1, Lin-28, Pax6b, cMyca/b (myelocytomatosis oncogene a/ b), and Oct4 (octamer-binding protein 4, also called POU class 5 homeobox 1) (Ramachandran et al., 2010a), and in mouse neural stem cells, let7 $b$ targets both NR2E1 (TLX, Nuclear Receptor subfamily 2, group E, member 1) and CyclinD (Zhao et al., 2010b). Because let-7 expression is reduced in the zebrafish retina through at least 7 days after stab wounds (Ramachandran et al., 2010a), its absence might have long-lasting influences on cell fate decisions of proliferating retinal progenitors. For example, even when only certain retinal cells are targeted by the lesion (such as photoreceptors in a light damage paradigm), some of the retinal progenitors differentiate into non-photoreceptor neurons (Bernardos et al., 2007; Lenkowski et al., 2013); similarly, after surgical excisions, Müller glia in the surrounding, undamaged retina proliferate to produce retinal progenitors that differentiate into cone photoreceptors and interneurons in regions that were not damaged (Cameron, 2000). Further studies into the regulation and precise role of microRNAs during retinal regeneration could elucidate RNA-based manipulations that might translate the success of regeneration in adult zebrafish retina to the mammalian retina.

Other bHLH transcription factors known to regulate neuronspecific differentiation during development are also expressed during retinal regeneration, namely neuroD and neurogenin1 (ngn1), both of which are important in the developing retina (Korzh et al., 1998). NeuroD induces cell cycle exit and rod photoreceptor fate while suppressing Müller glial fate, and morpholino-mediated knock-down of NeuroD inhibits cell cycle exit (Ochocinska and Hitchcock, 2009). In the damaged adult retina, neuroD is not expressed in Müller glia or in the earliest progenitors, but is expressed later in photoreceptor progenitors from 3 to 7 days after light onset in a chronic light lesion (Thomas et al., 2012b). Further studies are needed to determine if neuroD controls cell cycle exit and rod photoreceptor fate during regeneration similar to development. Ngn1 is expressed in immature Müller glia during development, but not in the uninjured adult retina (Thummel et al., 2008a). In a chronic light lesion paradigm, Ngn1 protein is reexpressed in neurogenic clusters in both PCNA-positive and PCNA-negative cells, and subsets of Müller glia continue to express Ngn1 as late as 17 days after light damage (Thummel et al., 2008a). Again, further studies are needed to uncover the contributions of Ngn1 to retinal regeneration.

\subsection{Notch and Wnt signaling pathways}

Two other major signaling pathways - Notch and Wnt - have been studied in the context of retinal development, maintenance, and regeneration. In developing neuroepithelia of the brain and retina, in both fish and mammals, the timing of Notch signaling determines whether this pathway will maintain cells in a progenitor state, promote cell cycle exit, or promote and maintain glial identity (for example, see Bernardos et al., 2005; Chapouton et al., 2010; Del Bene et al., 2008; Del Debbio et al., 2010; Furukawa et al., 2000; Grandbarbe et al., 2003; Jadhav et al., 2006a,b; Murciano et al., 2002; Nelson et al., 2011; Ohata et al., 2011; Scheer et al., 2001; Surzenko et al., 2013). Additionally, it has been known for many years that Notch signaling components are up-regulated in the regenerating goldfish retina (Sullivan et al., 1997), and more recently the same was demonstrated in the zebrafish retina (Raymond et al., 2006). In an analysis of gfap:EGFP-labeled Müller glia and glial-derived progeny after an acute light lesion, all Notch signaling genes that were annotated in the microarray decreased up to three-fold through $16 \mathrm{hpl}$, after which a subset - deltaC, her9 (hairy-related 9), her4.2, jagged2 - increased through 36 hpl (Qin et al., 2009). The early sample times in this analysis $(8,16$, and $24 \mathrm{hpl}$ ) represent Müller glia that have not yet divided, whereas the later sample (36 hpl) includes neuronal progenitors, so the temporal regulation of Notch signaling components could reflect differences between Müller glia and progenitors. In whole retina lysates, jagged $1 a$ and deltaD ligands show decreased expression through at least 4 days of constant light; deltaA, deltaB, and several her/hes downstream target genes may initially be down-regulated but their expression then increases slowly, and notch receptors are differentially regulated, with notch 3 significantly up-regulated at 3 days of constant light damage (Kassen et al., 2007). Attempts to assess the function of Notch signaling have used the $\gamma$-secretase inhibitor, DAPT, which increases the zone of proliferation around stab wounds, inhibits insm1a expression, and potentiates the upregulation of ascl1a, hb-egfa, and egfr; conversely, constitutive activation by overexpression of the Notch intracellular domain (NICD) decreases expression of ascl1a, hb-egfa, and pax6b (Gorsuch and Hyde, 2013; Ramachandran et al., 2012; Wan et al., 2012). Similarly, post-natal mouse retinas treated with DAPT express higher levels of Ascl1a in Müller glia and lower levels of the Müller glial marker CyclinD3, but the competence to respond is gone by post-natal day 14 (Nelson et al., 2011).

In the embryonic zebrafish retina, differential levels of expression of notch receptors, delta ligands, and the Notch target gene her4 suggest a gradient of Notch signaling in the retinal neuroepithelium, with higher levels apical than basal; the apical-basal translocation of the nuclei of neuroepithelial retinal progenitor during IKNM exposes them to this gradient (Del Bene et al., 2008). Time-lapse imaging revealed that neuroepithelial progenitors divide either symmetrically (both daughters remain proliferative), or asymmetrically (one daughter exits the cell cycle to differentiate as a neuron). Nuclei that translocate farthest basally (where they may experience reduced Notch signaling) tend to have asymmetric, neurogenic divisions that generate post-mitotic daughter cells (Baye and Link, 2007). In the adult zebrafish brain, radial glia that express glial markers - S100 $\beta$, BLBP, and the gfap:GFP reporter and function as neural stem cells are maintained in a quiescent state by induction of Notch signaling from neighboring progenitor cells (Chapouton et al., 2010). As discussed in Section 4.2.3, when Müller glia are activated after a retinal injury, they undergo IKNM and divide asymmetrically at the apical surface to generate a proliferative neurogenic progenitor; the Müller glia nucleus then translocates and the progenitor daughter cell migrates basally into the INL. Therefore, as they reenter the cell cycle, the Müller nucleus may be exposed to Notch signaling gradients along the apical-basal axis. Although a role for Notch signaling gradients in the regenerating adult retina has not been studied directly, we speculate that an initial reduction of Notch signaling would allow Müller glia to dedifferentiate and reenter the cell cycle and neuronal progenitors to express pro-neural genes. Subsequent activation of Notch signaling in Müller glia by the associated neuronal progenitors could then reestablish glial identity, as well as regulate cell cycle exit and cell fate in the neurogenic cluster.

Wnt/ $\beta$-catenin signaling is likewise critical to successful retinal regeneration in both larval (Meyers et al., 2012) and adult zebrafish (Ramachandran et al., 2011). Microarray studies have shown that in the ONL, levels of wnt8a decrease after acute light lesion (Craig et al., 2008), and in Müller glia, expression of the inhibitor $d k k 1$ (dickkopf, WNT signaling pathway inhibitor 1) decreases while the receptor $f z d 8 c$ (frizzled homolog $8 c$ ) increases through $36 \mathrm{hpl}$ (Qin et al., 2009). A closer look at Wnt signaling in regeneration reveals that $\beta$-catenin accumulates specifically in proliferative Müller glia and neuronal progenitors, and genetic or chemical inhibition of Wnt signaling prevents the proliferative response to retinal damage in larval and adult fish (Meyers et al., 2012; Ramachandran et al., 2011). After pharmacological hyperactivation of Wnt signaling following acute light lesions in larval zebrafish, newly generated 
cells all remain in the ONL and Müller glia are lost, suggesting that progenitor cell fate is altered, possibly through symmetric division of activated Müller glia (Meyers et al., 2012).

While activated Wnt signaling is necessary for larval photoreceptor regeneration, hyperactivation of the pathway via immersion of larvae in a synthetic Wnt agonist is not sufficient to induce proliferation in the uninjured retina (Meyers et al., 2012). In contrast, intraocular injection of the same agonist in the adult is sufficient to induce critical regeneration genes, such as ascl1a and lin28, and generation of several types of retinal neurons (Ramachandran et al., 2011). Simultaneous stab wounding of the retina and injection of Wnt agonists increases the zone of proliferation around the lesion, and this response is mediated by both lin28-dependent and -independent mechanisms downstream of ascl1a (Ramachandran et al., 2011). These effects in the adult are likely mediated by the specific Wnt pathway members whose expression changes in activated Müller glia, including wnt2ba, wnt4a, wnt8b, fzd2, fzd3, fzd8c, dkk1a, and dkk1b (Qin et al., 2009; Ramachandran et al., 2011). Furthermore, Ascl1a indirectly inhibits expression of the Wnt antagonist $d k k 1 b$, and their expression patterns are mutually exclusive after stab wounds (Ramachandran et al., 2011). These regulatory interactions may be mediated by insm $1 a$, as Insm 1 a can directly bind the $d k k 1 b$ promoter and insm $1 a$ morpholino-mediated knock-down leads to increased expression of both ascl1 $a$ and $d k k 1 b$ (Ramachandran et al., 2012). Interestingly, in laser-injured retinas of transgenic adult mice that have a greater and more prolonged increase of Wnt signaling (caused by loss of the negative Wnt regulator, Axin2), Müller glia show increased proliferation and generate rhodopsin-positive cells (Liu et al., 2013).

Notch and canonical Wnt signaling may have complementary roles in regeneration. Mammalian Müller glia divide and express opsin when cultured with Notch and Wnt ligands (Jagged1 and Wnt3a, respectively), and these effects are stronger when both pathways are activated (Das et al., 2006; Del Debbio et al., 2010). Similarly, in the damaged adult zebrafish retina, activation of both pathways is downstream of HB-EGF signaling (Wan et al., 2012). In situ hybridization to detect Notch signaling components suggest that Müller glia may be adjacent to cells expressing delta ligands (Wan et al., 2012). However, in situ signals are too diffuse to reliably distinguish individual cells within the neurogenic cluster, and further studies are needed to determine if components of Notch and Wnt signaling are differentially expressed among retinal progenitors and Müller glia, as well as along the apical-basal axis of the Müller glia, during retinal neuron regeneration. Similar to Notch signaling, gradients of canonical Wnt signaling may also be important for asymmetric divisions in Müller glia during retina regeneration; in ESCs, Wnt signaling orients mitotic spindles, leading to an asymmetric division in which the daughter cell with lower Wnt signaling is more differentiated (Habib et al., 2013).

\subsection{Other stem cell factors (Sox2, BLBP)}

Other markers of neurogenic stem cells are expressed in the regenerating adult zebrafish retina, although their role in regeneration has yet to be investigated. For example, in the mammalian brain, Sox2 (SRY-box containing gene 2 ) is expressed in both slowly dividing radial glia and in the transient amplifying progenitors they produce (as reviewed in, Hsieh, 2012). Additionally Sox2 has a welldefined role in generating iPSCs from mammalian embryonic fibroblasts (Takahashi et al., 2007; Takahashi and Yamanaka, 2006). In the postnatal mouse retina, Sox2 is critical for establishing Müller glial identity via Notch signaling and quiescence via an alternative mechanism (Surzenko et al., 2013). As with Pax6, mammalian Müller glia express the stem cell marker Sox2 (Roesch et al., 2008). In zebrafish, expression of sox2 in activated Müller glia increases 2-fold by $8 \mathrm{~h}$ after an acute light lesion (Qin et al., 2009). In whole retina lysates collected after stab wounds, sox2 expression increases slightly at $2 \mathrm{dpl}$, but peaks at $4 \mathrm{dpl}$ and is undetectable at $7 \mathrm{dpl}$ (Ramachandran et al., 2010a). The differences between these studies are likely due to a combination of lesion paradigm and detection methods. Nevertheless, the sustained expression of Sox2 after stab wounds suggests that neurogenic progenitors continue to express sox2 after Müller glia have redifferentiated.

Expression of BLBP, a fatty acid binding protein, is associated with non-dividing radial glia (Chapouton et al., 2010) and multipotent progenitors in the adult zebrafish brain (Chapouton et al., 2010; Hartfuss et al., 2001), as described above in Section 2.2, and it is expressed by immature and reactivated Müller glia in the adult zebrafish retina, as described above in Section 4.2.2. BLBP expression in Müller glia increases rapidly, within $1 \mathrm{~h}$ after an acute light lesion (Nagashima et al., 2013), remains elevated at 14 days in the lesioned area relative to surrounding unlesioned areas of retina (Lenkowski et al., 2013), then is down-regulated by 28 days (J.L., unpublished data). Therefore, BLBP likely has an important but yet unexplored role throughout retinal neuron regeneration, not solely in the transient dedifferentiation of Müller glia immediately after retinal damage. The fatty acid docosahexaenoic acid (DHA) is the main binding partner for BLBP (Xu et al., 1996). Müller glia can increase survival of neurons in culture in part because they can transfer DHA to the neurons (Politi et al., 2001), and DHA can induce neural stem cells to differentiate into neurons by downregulating Hes 1 and up-regulating NeuroD (Katakura et al., 2009). Therefore, BLBP may be involved in regulation of cell cycle, cell survival, and cell fate during regeneration.

\subsection{Cell adhesion and migration (N-cadherin, Alcama, Galectin, MMPs)}

While many research groups have shown the importance of secreted factors and intracellular signaling pathways for successful regeneration of retinal neurons, little attention has been given to the extracellular milieu within the regenerating retina. The presence and importance of particular extracellular proteins is now becoming more apparent, and the type of proteins present may depend on the nature of the damage. $\mathrm{N}$-cadherin (cdh2) is strongly expressed in the stem cell niche in the CMZ in adult fish and is upregulated in neurogenic clusters after retinal damage by intense light, neurotoxin (ouabain), or stab wounds (Liu et al., 2002; Nagashima et al., 2013; Raymond et al., 2006). In fact, $c d h 2$ likely has a specific role in regeneration since it is up-regulated in Müller glia within $8 \mathrm{~h}$ after an acute light lesion, while other cadherins are down-regulated (Qin et al., 2009). After stab wounds, Cdh2 protein increases by $2 \mathrm{dpl}$, and R-cadherin (Cdh4) after 5-7 dpl (Liu et al., 2002). In fish heterozygous for a semi-dominant mutant allele of $c d h 2$, Müller glia nuclei still exhibit IKNM after damage, but basal migration of retinal progenitor cells is blocked; because progenitors remain at the apical surface, they successfully regenerate photoreceptors destroyed by light lesion, whereas regeneration of ganglion cells is significantly reduced (Nagashima et al., 2013).

Other extracellular proteins are likely important for cell adhesion and migrational cues in neurogenic clusters of retinal progenitor cells. A novel stem cell marker, Alcama (Activated leukocyte cell adhesion molecule a, a member of the immunoglobin superfamily), was identified in progenitor cells in the CMZ and is transiently expressed by injury-induced Müller glia, and not progenitors, after acute light lesion (Nagashima et al., 2013). Alcama expression may be related to the function of $\mathrm{N}$-cadherin in mediating cell-cell adhesion in the neurogenic clusters (Nagashima et al., 2013). Human ALCAMA can recruit N-cadherin and $\beta$-catenin in cancer cells and it promotes activity of matrix 
metalloproteinases (MMPs) in vitro (Jannie et al., 2012). We speculate that Alcama may play a similar role related to migration of retinal progenitors and reestablishment of cell-cell junctions in the regenerating zebrafish retina.

NCAM is expressed in Müller glia in the unlesioned adult zebrafish retina, but only on the Müller glial processes in the ONL, where it has been suggested to provide directional migratory cues and a microenvironment for undifferentiated rod precursors (Kustermann et al., 2010). Transcription of ncam is decreased in Müller glia through $36 \mathrm{~h}$ after acute light lesion (Qin et al., 2009), but transcription levels increase slowly during a chronic light lesion, as measured by microarray analysis of whole retina homogenates (Kassen et al., 2007). The extracellular Tenascin proteins inhibit axon outgrowth in optic nerve regeneration (Faissner and Kruse, 1990); tenascin C (tnc) can be regulated by TGF $\beta$ signaling (Chablais and Jaźwińska, 2012) and itself regulates the Müller glial response to growth factors (Besser et al., 2012). Tenascins are also differentially regulated after retinal lesions. Microarray data suggest that tenascin $R$ is down-regulated in Müller glia through $36 \mathrm{~h}$ after an acute light lesion (Qin et al., 2009), and tnc is upregulated at $96 \mathrm{~h}$ in the whole retina during chronic light lesions (Kassen et al., 2007).

The zebrafish homolog of Galectin-1 (drgal1-l2) was the first molecule characterized with the ability to modulate photoreceptor regeneration but is not expressed in the developing zebrafish retina (Ahmed et al., 2004 9612; Craig et al., 2010). Galectin is a secreted protein, and Peter Hitchcock and colleagues (Craig et al., 2008) suggest that its role in regeneration is related to its ability to bind proteins in the extracellular matrix (ECM). dgral1-l2 is up-regulated within $12 \mathrm{~h}$ after acute light damage (Craig et al., 2008), and it is expressed in microglia, proliferating Müller glia, and Müller gliaderived progenitors through $7 \mathrm{dpl}$ (Craig et al., 2010). Morpholino-mediated knock-down of Drgal1-L2 significantly reduces the number of regenerated rod, but not cone, photoreceptors at $7 \mathrm{dpl}$ (Craig et al., 2010).

The obvious requirement for remodeling of both cell-cell adhesion and the ECM to support progenitor cell migration and differentiation of regenerated neurons has not yet received much attention. A number of enzymes that remodel the extracellular environment are up-regulated after retinal lesions: $\operatorname{mmp9}$ (matrix metalloproteinase 9, gelatinase B), mmp13 (also known as collagenase 3), and timp2 (tissue inhibitor of metalloproteinase 2) all increase after a variety of lesions (Cameron et al., 2005; Kassen et al., 2007; Qin et al., 2009). MMP9 is regulated by signals that include MAPK/ERK1/2, glycogen synthase kinase $3 \beta$ (an effector of the Wnt/ $\beta$-catenin pathway), TNF $\alpha$, hypoxia, and histamines (Vandooren et al., 2013), so it is not surprising that its expression increases dramatically in different types of retinal lesions. MMPs have known functions in healthy and diseased retinas (Sivak and Fini, 2002) and regulate the behavior of neural progenitor cells (Barkho et al., 2008). Because soluble HB-EGF can rescue proliferation in adult zebrafish Müller glia after stab wounds and treatment with a pan-MMP inhibitor inhibits retina regeneration (Wan et al., 2012), it is apparent that remodeling and signaling in the extracellular matrix of the retina is critical for successful retinal neuron regeneration in adult zebrafish.

\subsection{Summary and overview}

It is clear that a complex network of signals derived from dying neurons, reactive microglia, Müller glia, and potentially other cellular elements, is critical for a successful regenerative response in the adult zebrafish retina. The idea that regeneration may partially recapitulate development has stimulated investigators to characterize many factors known to be essential for retinal developmental, but other promising signaling pathways and transcription factors have not yet been well studied in the context of retinal regeneration. Several retinal lesion paradigms have been developed to study regeneration in teleost fish - surgical ablation, neurotoxin injection, acute and chronic light lesions, stab wounds, and targeted genetic ablation of specific types of retinal neurons and each of these lesion paradigms likely induces a variety of molecular responses. For example, break-down of the blood-retinal barrier (a consequence of some but not all lesion methods) can expose the retina to additional cytokines, blood components, and other factors, which in the mammalian retina can tip the balance between a "conservative", non-proliferative and a "massive", proliferative Müller glial response to damage (Bringmann et al., 2009). Distinct signaling pathways may also be activated depending on which type of retinal neurons are damaged or destroyed. While several lesion paradigms ablate specific retinal neurons whereas others cause non-selective damage, discussions of experimental findings have sometimes neglected to consider these differences when interpreting the subsequent regenerative response.

While many identified genes and signaling pathways reviewed here play important roles in the regulation of Müller glia-based retinal neuron regeneration, it is certain that interactions among these various molecular mechanisms are necessary to orchestrate the appropriate responses to repair the damage (Fig. 4). For example, downstream targets of Notch and Wnt/ $\beta$-catenin converge to activate a regenerative response in zebrafish Müller glia (Wan et al., 2012) and to induce neuronal markers in mammalian Müller glia (Das et al., 2006; Del Debbio et al., 2010). In addition, direct protein-protein interactions have been shown to mediate cross-talk between Jak-Stat/Notch and TGF $\beta /$ Notch in mammalian neural stem cells and brain tumors (Aigner and Bogdahn, 2008; Kamakura et al., 2004). Several additional clues to potentially relevant interactions can be found in recent successful reports of generating retinal progenitors in vitro from rodent Müller glial cells, ESCs, or iPSCs (Ikeda et al., 2005; Lamba et al., 2006; Pollak et al., 2013) and the even more remarkable demonstration of the self-organizing morphogenesis of optic cups from ESCs and iPSCs in three-dimensional cultures (Eiraku et al., 2011; Lamba et al., 2009a; Osakada et al., 2008). Some of the relevant pathways include many that were discussed above: 1) Factors that inhibit TGF $\beta$ signaling, such as Tgif1, are enhanced in human ESCs in an 'undifferentiated state', as are factors that activate Wnt and FGF signaling (Sato et al., 2003). 2) A small molecule inhibitor of TGF $\beta$ signaling can replace Sox2 in reprogramming fibroblasts to become iPSCs (Ichida et al., 2009). 3) Various ECM components, such as laminin, entactin, and synthetic basement membrane, improve in vitro generation of retina from ESCs when the TGF $\beta$ ligand Nodal is also included in the media (Eiraku et al., 2011). 4) When immortalized Müller glia are cultured with ECM and FGF or RA, they form neurospheres that express progenitor and post-mitotic neuron markers, and these cells migrate into and express appropriate markers of all retinal layers when grafted into the retina (Lawrence et al., 2007).

Since regeneration must, at least to some fundamental degree, recapitulate development - including cell amplification, cell fate determination, cell patterning, and morphogenesis - it is not surprising that so many of the major developmental signaling mechanisms that control gene expression and cell behavior are resurrected to rebuild the damaged retinal tissue. The future challenge for the field will be to understand how this myriad of molecular and cellular 'threads' is woven together in a tightly regulated spatial-temporal biological network to achieve functional retinal regeneration. Future studies of zebrafish retinal regeneration should address not only the molecular and cellular processes that modulate the behavior and fate of reactive Müller glia and 
proliferating retinal progenitors, but also consider the regenerative outcome, an endpoint not addressed in many studies. With a deeper and more comprehensive understanding of repair mechanisms in the teleost fish retina, translational studies aimed at promoting functional regeneration in the human retina will have a better chance of success.

\section{Gliosis versus neurogenesis - questions for the future}

\subsection{How do fish and mammalian Müller glia differ in their response to injury?}

For many researchers - those who study retinal regeneration in fish and those who follow this field - the key question is: Why do Müller glia in fish retinas exhibit such a robust ability to generate retinal neurons while Müller glia in human retinas do not? Useful insights into this question can be gained by comparing injury responses in fish and mammalian Müller glia. Two reviews published in previous issues of this journal by Andreas Bringmann and colleagues provide comprehensive summaries of the exceedingly complex responses of Müller glia to retinal injury (Bringmann et al., 2009, 2006). Briefly, astrocytes and related cells (e.g., Müller glia) respond to injury or disease in the nervous system by undergoing reactive gliosis, which has both positive or neuroprotective and negative or detrimental effects. The neuroprotective effects of gliosis in Müller glia reflect a cellular attempt to limit tissue damage and neuronal loss, and include buffering of potassium, uptake of glutamate, release of antioxidants, release of Nitric Oxide (NO), phagocytosis, and release of neurotrophic factors. In contrast, detrimental effects may increase neuronal death, result in glial scarring, and include release of proinflammatory cytokines such as TNF, excess production of NO, and enhanced production of polyamines that coactivate NMDA receptors. Importantly, the same gliotic response or factor (e.g., NO) can have both positive as well as negative effects, depending on the time, duration, or amplitude of the response. Chronic overstimulation or dysregulation of gliosis is typically associated with detrimental effects.

The characteristic cellular responses of gliosis include: hypertrophy, increases in intermediate filaments (in particular, GFAP), and, if the damage is severe and/or prolonged, proliferation and cell migration leading to formation of glial 'scars'. The molecular features of gliosis reflect a stress response with expression of immediate early response genes, such as c-fos and c-jun (for details and references the reader is referred to Bringmann et al., 2009). Signaling pathways that are activated in, and factors released by, reactive mammalian Müller glia include many of those discussed above in Section 5, such as: ERK1/2, JAK/STAT, TGFB/SMAD2/3, HBEGF, MMP-9, IL-6, TNF, and Galectins. In a recent review, Magdalena Götz and colleagues compared signaling pathways activated during reactive gliosis in astrocytes of the mammalian CNS with signaling that regulates adult neural stem cells (Robel et al., 2011). Mammalian adult neural stem cells resemble radial glia and are located in two distinct niches in the CNS, and the same signal can lead to different outcomes in reactive astrocytes in the brain parenchyma compared with the neural stem cells. Perhaps the most striking example is the effect of BMP (a member of the TGF $\beta$ superfamily): In astrocytes after injury, the downstream mediator of BMP, SMAD1, interacts cooperatively with STAT3 to promote GFAP expression and glial fate, whereas in the neural stem cell niche in the subependymal zone of the lateral ventricle, BMP signaling promotes self-renewal and neurogenesis. The relatively low level of STAT signaling in the neurogenic regions may account for this differential response (Robel et al., 2011).

Reactive mammalian Müller glia show signs of dedifferentiation associated with cell cycle reentry, including down-regulation of specific markers such as CRALBP, GS, and carbonic anhydrase (CA), up-regulation of progenitor markers like Pax6, and alterations in ionic currents that depolarize Müller glia. A recent study by Edward Levine, Robert Marc, and colleagues clearly demonstrated that Müller glia can retain their normal, metabolic support functions even while proliferating (Vazquez-Chona et al., 2011). This study showed that conditional ablation of the cyclin-dependent kinase inhibitor, $p 27^{k i p 1}$, with the tamoxifen-regulated CreER-loxP system, produced a gliotic response in retinal Müller glia - hypertrophy, increased expression of GFAP, reentry into the cell cycle, and apically displaced nuclei - in the absence of retinal injury. Müller glial markers Sox9 and GS co-localized with BrdU after a six-week chase, but glial scars were not formed, and computational molecular phenotyping of metabolomic signatures together with electrophysiological and behavioral measures indicated that these reactive Müller glia maintained retinal homeostasis to preserve visual function.

In summary, with the exception of glial scar formation, virtually all other features of gliosis that have been described in mammalian Müller cells appear to be shared by Müller glia in the injured fish retina. The difference, then, lies in the fate of the cells that result from proliferation of Müller glia.

\subsection{What cellular and molecular signals activate a stem-cell-like (i.e., asymmetric self-renewing) division of Müller glia rather than inducing glial scar formation?}

Severe retinal insults in mammals can trigger a "massive" or proliferative gliosis, characterized by features including uncontrolled symmetric cell division and migration of Müller glia, transdifferentiation of Müller glia into contractile myofibrocytes, and formation of glial scars within the retina and on the subretinal or epiretinal surfaces (Bringmann et al., 2009, 2006). In the severely injured fish retina, reactive Müller glia exhibit all the hallmarks of proliferative gliosis except they do not form glial scars; instead their proliferation is tightly controlled and limited, they retain their epithelial identity, and they do not undergo an epithelialmesenchymal transformation associated with cell migration.

Although zebrafish Müller glia are differentiated cells, in the uninjured, growing retina, Müller glia are the source of rod precursors, and therefore exhibit the hallmarks of somatic stem cells quiescent or slowly cycling with asymmetric, self-renewing mitotic divisions. Thus, fish Müller glia may be primed to produce neurogenic retinal progenitors by asymmetric division in response to homeostatic signals to generate lineage-restricted rod progenitors or to generate multipotent retinal progenitors in response to injury/stress signals. To date, investigations of retinal regeneration have concentrated on injury-induced changes in Müller glia; this is a reasonable strategy, except that most of the observed changes in gene expression and signaling mechanisms (e.g., Stat3, HB-EGF, TNF $\alpha$ ) may reflect reactive gliosis rather than neurogenesis. The transcription factors associated with a neurogenic program and necessary for regeneration of retinal neurons (e.g., Acsl1a, Pax6, Six3, NeuroD) must necessarily drive cell fate decisions in the Müller glial-derived retinal progenitors, and not in the Müller glial stem cells themselves. To date it has proved technically difficult to explicitly identify Müller glial cells versus glial-derived retinal progenitors in neurogenic clusters in histological sections or in cells isolated by FACS with lineage-tracing markers. This limitation perhaps accounts for some of the contradictory data in the literature.

Time-lapse microscopy of injury-induced Müller glia in the zebrafish retina in vivo would offer important insights and direct evidence for their stem cell properties. Methods developed to visualize genetically targeted fluorescent reporters in the developing retina of embryonic zebrafish, notably by Brian Link, William 
Harris, Rachel Wong, and their respective colleagues, have provided invaluable insights into the mechanics and kinetics of cell proliferation and the lineage of retinal progenitors in embryos (Agathocleous and Harris, 2009; Baye and Link, 2007; He et al., 2012; Jusuf et al., 2013; Norden et al., 2009; Suzuki et al., 2013; Williams et al., 2013). Two limitations in applying these techniques to study retinal regeneration in adult zebrafish are: 1) Melanin pigmentation in the RPE and iridophores on the external surface of the eye both obscure the underlying neural retina from view. 2) Immobilizing and maintaining animals viable for the long periods required for time-lapse imaging is challenging. One solution is to employ pigmentation mutants (albino; roy) that block formation of both melanin (albino) and iridophores (roy), in combination with a regeneration model suitable for young larval fish (less than 1-week-old), which are small enough that gas exchange by diffusion is sufficient to maintain adequate oxygen tension during the prolonged immobilization required for time-lapse imaging. For example, Jeff Mumm and co-workers have recently developed time-lapse imaging methods that employ nitroreductase-metronidazole genetic targeting to ablate specific retinal cell types in albino; roy mutant larvae (Ariga et al., 2010; White and Mumm, 2013). An alternative would be to develop techniques for retinal explant or slice cultures; these methods have been extremely valuable for in vivo time-lapse imaging in the developing mammalian CNS (Kechad et al., 2012; Noctor et al., 2001, 2002; Wang et al., 2002a).

The process of asymmetric, self-renewing divisions has been well characterized in Drosophila, but is much less understood in vertebrates (Gotz and Huttner, 2005; Huttner and Brand, 1997; Zhong and Chia, 2008). Mediators of apical-basal epithelial polarity and mitotic spindle orientation are proposed to control the unequal distribution of cellular constituents that lead the daughter cells to adopt different fates. In the developing Drosophila nervous system, a key determinant of daughter cell fates is the endocytic adaptor protein Numb, which is asymmetrically inherited and antagonizes Notch signaling. Mammals have two Numb orthologs, Numb and Numb-like, but their relationship to asymmetric division and cell fate is complex and not understood. A recent study by Michel Cayouette, William Harris, and their colleagues used conditional knockout of Numb and Numb-like and clonal analysis in developing mouse retina to show that Numb activity is required for cell-cycle progression at early stages, but at later stages it is critical for terminal asymmetric cell divisions that generate two different retinal cell types, including, for example, a Müller glial cell and a rod photoreceptor (Kechad et al., 2012). With time-lapse microscopy of neonatal mouse retinal explants and retroviral-mediated expression of a Numb:Venus fusion protein, the authors demonstrated rapid temporal modulation of Numb localization, which maintains a polarized distribution for no more than 3040 min during the cell division cycle. Temporal regulation and dynamic oscillation of Notch signaling in coordination with specific cell behaviors is recognized as an important parameter in its mode of action (for example Shimojo et al., 2008), which further emphasizes the need for time-lapse microscopy with transgenic reporters to ascertain the roles of Notch signaling in retinal regeneration.

\subsection{Why do the Müller glial cell and its glial-derived retinal} progenitor have such different fates (i.e., the former retains its glial properties and exits the cell cycle while its daughter cell proliferates and differentiates into neurons)?

Differential Notch-Delta signaling is certainly involved in specifying the identity of Müller glia-derived progenitors as distinct from their parent Müller glia, although we are a long way from understanding the mechanisms. We know that components of the Notch pathway are expressed in Müller glia and up-regulated after injury, and that genetic or pharmacological manipulations interfere with glial-derived neurogenesis, as discussed above (Sections 3.2 and 5.5). However, the spatiotemporal resolution of the experiments performed to date provides little information about differential Notch signaling in Müller glia and glial-derived retinal progenitors.

Molecular genetic analyses of the Notch pathway in neuroepithelial cells in the developing zebrafish brain are beginning to provide some insights. Time-lapse imaging and clonal genetic mosaic analysis of radial glial progenitors in the developing zebrafish brain demonstrate that within a single lineage, selfrenewing divisions produce daughter cells with different apicalbasal positions and asymmetry of Notch activity (Dong et al., 2012). Modulation of Notch activity is mediated by differential inheritance of the ubiquitin E3 ligase Mind bomb (Mib), which promotes Notch activity by modulating endocytosis of Notch ligands; segregation of Mib to the apical daughter is dependent on Par3 (par-3 family cell polarity regulator), a component of the apical epithelial polarity complex. The interaction between Notch signaling and apical polarity was also revealed by an analysis of IKNM in neuroepithelial cells in the brain of zebrafish mosaic eyes (moe) mutants, in which the interaction between the Epb4115 (Erythrocyte membrane protein band 4.1-like 5) adaptor protein and the apical-basal polarity regulator, Crumbs (Crb), is disrupted; Crb directly interacts with and inhibits Notch signaling and Moe blocks this inhibition, contributing to the apical-basal gradient of Notch activity and apically restricted mitoses (Ohata et al., 2011). Future studies of retinal regeneration that investigate the dynamics of Notch signaling in proliferating Müller glia and their neurogenic progeny, and in relation to apical-basal polarity and position of progenitors, will provide important next steps in clarifying a role for Notch signaling in the fate of Müller gliaderived daughter cells.

\subsection{How does the physical microenvironment of Müller glia, in particular cell-cell adhesion and cell signaling as understood in the context of a stem cell 'niche', control the behavior of Müller glia?}

Müller glia proliferation in the fish retina is exquisitely responsive to homeostatic growth signals that dictate the rate of production of rod photoreceptors and is enhanced in response to retinal injury or cell loss. The Hippo pathway, first identified in Drosophila, has recently emerged as an important regulator of organ size homeostasis through its control of proliferation and apoptosis (Halder and Johnson, 2011; Yu and Guan, 2013; Zhao et al., 2010a). Vertebrate orthologs of the core components of the Drosophila Hippo pathway kinase cascade have been identified, as well as the effector of the pathway, the transcriptional coactivators YAP (Yes-Associated Protein) and its paralog, TAZ (Taffazzin). Signaling through the Hippo pathway results in the phosphorylation and inactivation of YAP/TAZ, which are sequestered in the cytoplasm. When dephosphorylated, YAP/TAZ translocate to the nucleus and activate genes that promote proliferation and block apoptosis. The core Hippo kinase cascade functions to control cell number and organ size during development and in pathological conditions; regulation of Hippo signaling is crucial for regeneration of the mammalian liver and its dysregulation is associated with cancers (Halder and Johnson, 2011; Zhao et al., 2010a).

Upstream regulators of the Hippo pathway in vertebrates are just beginning to be discovered, and include: 1) components of cell-cell junctions (adherens junctions and tight junctions) that mediate apical-basal epithelial polarity, 2) mechanical forces from 
the ECM modulated through the actin cytoskeleton, and 3) diffusible signals that act through G-protein-coupled receptors and Rho GTPases (Yu and Guan, 2013). The Hippo pathway senses epithelial cell density and inhibits cell proliferation through direct interactions of YAP/TAZ with apical junction proteins in the Crumbs complex (Varelas et al., 2010) and E-cadherin/ $\beta$-catenin regulation of the kinase cascade (Kim et al., 2011). When cell-cell junctions are disrupted, dephosphorylated YAP/TAZ translocate to the nucleus to activate target genes that promote proliferation. YAP/TAZ also regulate TGF $\beta$ signaling by controlling the nuclear localization of SMAD2/3; phosphorylated YAP/TAZ in the cytoplasm bind SMAD2/3, preventing cells from responding to TGF $\beta$ signaling (Varelas et al., 2010). The apical radial processes of Müller glia form highly specialized junctions with photoreceptors at the OLM, which represent a hybrid between adherens and tight junctions (Gosens et al., 2008), and Müller glia extend elaborate lateral processes that enwrap retinal neurons and their processes (Figs. 1 and 3). Because they literally touch all of the neurons, Müller glia are exquisitely well positioned as cell density sensors. Despite the obvious relationship between the cellular activities mediated by Hippo signaling, and the neurogenic responses of Müller glia, it is surprising that nothing is yet known about regulation of this pathway during retinal growth and regeneration.

In contrast to the permissive environment for neurogenesis in the fish retina, the mammalian retina presents a non-permissive environment for regeneration in that inhibitory ECM and cell adhesion molecules - chondroitin sulfate proteoglycans and the hyaluron-binding glycoprotein CD44 - are expressed on the surface of reactive Müller glia (for references see Bringmann et al. 2009). Although the characteristics of the ECM in and around the neurogenic clusters in the regenerating fish retina have not been examined, in the intact retina the permissive version of NCAM, PSA-NCAM, is expressed on the apical processes of Müller glia associated with on-going production of rod photoreceptors (Kustermann et al., 2010). The addition of PSA to NCAM can disrupt cell adhesion, and it has been proposed that the balance between PSA-NCAM, NCAM, and N-cadherin regulates the neurogenic niche in the brain (Gascon et al., 2010). The presence of PSA-NCAM is important for migration and survival of neuroblasts, and progressive down-regulation of PSA-NCAM is associated with neuronal differentiation once they reach their final location (for references see Gascon et al., 2010). The regulation of $\mathrm{N}$-cadherin is important for adhesion and migration of cells in the neurogenic cluster during retinal regeneration (Nagashima et al., 2013), but PSA-NCAM has not been investigated in this process.

\subsection{Is 'reprogramming' of Müller glia an accurate description of the} regenerative response, and should this be the target for therapeutic approaches?

"Reprogramming" is a popular term used in stem cell research to describe somatic cells that are forced to undergo cell type switching and achieve a pluripotent state with the consequent loss of differentiated cell identity (Plath and Lowry, 2011; Takahashi and Yamanaka, 2006). Earlier uses of this term referred to the changes in differentiated tissue that result in epimorphic regeneration, for example, of amphibian limbs (Brockes and Kumar, 2002). Recent genetic cell fate mapping studies with Cre-ER/loxP techniques applied to regenerating tails of Xenopus tadpoles and limbs of salamanders established that differentiated cells in the stump adjacent to the wound dedifferentiate (reprogram) to become lineagerestricted progenitors, reenter the cell cycle, and form a regeneration blastema (Jopling et al., 2011; Tanaka and Reddien, 2011). Genetic lineage tracing tools have similarly established that dedifferentiated cells are the source of regenerated tissue following amputation of zebrafish heart and fin (Choi and Poss, 2012; Lee et al, 2005; Jopling et al., 2011).

Although retinal regeneration in zebrafish has also been attributed to reprogramming - in this case reprogramming of Müller glia (Knapp and Tanaka, 2012) - this characterization is misleading because of a sharp distinction in the requirements for repair of a damaged retina versus an amputated limb, fin, tail, or heart. In the later cases, rebuilding lost tissues requires de novo regrowth of the organ. Newly regenerated tissue is added onto the residual structure by progenitors in the regeneration blastema on the proximal side, which differentiate and reorganize into patterned tissues, while proliferating cells on the distal side of the blastema continue to produce more cells until the missing parts are restored. In contrast, regeneration of retinal neurons is not accomplished by de novo growth, but rather by selective restoration of cells within the context of the existing tissue architecture. A similar scenario plays out during regeneration after brain lesions in zebrafish, where radial glia divide asymmetrically to generate neuronal progenitors (neuroblasts) that differentiate into neurons to repair the damage (Grandel and Brand, 2013; Kizil et al., 2012; Kroehne et al., 2011; Zupanc and Sirbulescu, 2011). In the CNS of fishes, radial glia and Müller glia divide asymmetrically to selfrenew and generate neuronal progenitors for both growth and homeostasis, as well as functional repair and regeneration. Thus, radial glia and Müller glia are multipotent stem cells that do not require 'reprogramming' in the classic sense.

The demonstrable neurogenic potential of glial cells in the adult vertebrate CNS (a potential that is largely unrealized in mammals but robustly operative in fishes) has encouraged attempts to 'reprogram' mammalian glial cells into neuronal progenitors for the purpose of repairing and restoring neural damage. Taking a lead from the iPSC field, several investigators have used viral-mediated expression of neural determination factors, such as Pax6, Ngn2, or Acsl1, to reprogram astrocytes in vitro (reviewed in Robel et al., 2011). Recently, for example, Thomas Reh and colleagues showed that viral-mediated expression of Ascl1 in dissociated mouse Müller glia or in retinal explants reprogrammed them into retinal progenitors that up-regulated many progenitor genes implicated in Müller-glia based regeneration in the zebrafish retina, including Hes5, Insm1, Fabp7, Ngn2, Olig2, and then differentiated into cells with neuronal properties, albeit not very efficiently (Pollak et al., 2013). These authors also showed that Ascl1 remodels chromatin to an active state at the promoters of progenitor genes, although they speculated that epigenetic restrictions may have prevented more complete reprogramming. A goal of this study, and many others like it, is to obtain a sufficient quantity of retinal progenitors for transplantation in order to treat retinal degenerative diseases. The alternative gene therapy approach, i.e., to reprogram endogenous Müller glia in situ, is more equivalent to evoking an endogenous regenerative response in the retina, but Müller glial reprogramming would have to overcome the serious and therapeutically unacceptable consequences of losing Müller glia by converting them into neurons.

Müller glia in the fish retina are not reprogrammed to regenerate neurons. They are exquisite cellular sentinels of the physiological and mechanical status and neural activity in the retina, and they are always poised to reenter the cell cycle to generate a retinal progenitor when growth or injury signals call for more neurons. As discussed above, some of the important extracellular and intracellular signaling pathways and gene networks that regulate Müller glial-dependent neurogenesis are emerging, although certain aspects of this process have received much less attention. These understudied topics include cellular and molecular mechanisms of cell adhesion and cell polarity, cell density sensors, asymmetric 
division, and differential fate of daughter cells. Another key area for future research is to better understand the epigenetic status of Müller glial stem cells in the zebrafish retina, and how chromatin modifications mediate changes in gene expression in response to homeostatic and injury signals. As documented in this review, retinal regeneration research in adult zebrafish rests on a solid foundation, and further studies will paint a more complete picture by addressing the many remaining mechanistic gaps and unanswered questions. An important goal is to better inform translational studies aimed at harnessing the regenerative potential of human Müller glia and creating a favorable microenvironment to allow for functional clinical repair of the damaged or diseased retina.

\section{Acknowledgments}

We apologize to those researchers whose contributions could not be cited due to limitations of space and scope for this review. We would like to thank Peter Hitchcock for helpful comments on the manuscript and many other colleagues for stimulating discussions. P.A.R. has published previously as P.R. Johns.

\section{References}

Abdipranoto-Cowley, A., Park, J.S., Croucher, D., Daniel, J., Henshall, S., Galbraith, S., Mervin, K., Vissel, B., 2009. Activin A is essential for neurogenesis following neurodegeneration. Stem Cells 27, 1330-1346.

Adler, R., Raymond, P.A., 2008. Have we achieved a unified model of photoreceptor cell fate specification in vertebrates? Brain Res. 1192, 134-150.

Adolf, B., Chapouton, P., Lam, C.S., Topp, S., Tannhauser, B., Strahle, U., Gotz, M., Bally-Cuif, L., 2006. Conserved and acquired features of adult neurogenesis in the zebrafish telencephalon. Dev. Biol. 295, 278-293.

Agathocleous, M., Harris, W.A., 2009. From progenitors to differentiated cells in the vertebrate retina. Ann. Rev. Cell Dev. Biol. 25, 45-69.

Ahlbert, I.B., 1976. Organization of the cone cells in the retinae of salmon (Salmo salar) and trout (Salmo trutta trutta) in relation to their feeding habits. Acta Zool. 57, 13-35.

Ahmad, I., Del Debbio, C.B., Das, A.V., Parameswaran, S., 2011. Müller glia: a promising target for therapeutic regeneration. Investig. Ophthalmol. Vis. Sci. 52, 5758-5764.

Ahmed, H., Du, S.J., O'Leary, N., Vasta, G.R., 2004. Biochemical and molecular characterization of galectins from zebrafish (Danio rerio): notochord-specific expression of a prototype galectin during early embryogenesis. Glycobiology $14,219-232$.

Aigner, L., Bogdahn, U., 2008. TGF-beta in neural stem cells and in tumors of the central nervous system. Cell Tissue Res. 331, 225-241.

Ali, M.A., 1964. Stretching of the retina during growth of salmon (Salmo salar), Growth 28, 83-89.

Allison, W.T., Barthel, L.K., Skebo, K.M., Takechi, M., Kawamura, S., Raymond, P.A., 2010. Ontogeny of cone photoreceptor mosaics in zebrafish. J. Comp. Neurol. 518, 4182-4195.

Alvarez-Buylla, A., Garcia-Verdugo, J.M., Tramontin, A.D., 2001. A unified hypothesis on the lineage of neural stem cells. Nat. Rev. Neurosci. 2, 287-293.

Amato, M.A., Arnault, E., Perron, M., 2004. Retinal stem cells in vertebrates: parallels and divergences. Int. J. Dev. Biol. 48, 993-1001.

Ariga, J., Walker, S.L., Mumm, J.S., 2010. Multicolor time-lapse imaging of transgenic zebrafish: visualizing retinal stem cells activated by targeted neuronal cell ablation. J. Vis. Exp., e2093.

Avanesov, A., Malicki, J., 2004. Approaches to study neurogenesis in the zebrafish retina. Methods Cell Biol. 76, 333-384.

Barkho, B.Z., Munoz, A.E., Li, X., Li, L., Cunningham, L.A., Zhao, X., 2008. Endogenous matrix metalloproteinase (MMP)-3 and MMP-9 promote the differentiation and migration of adult neural progenitor cells in response to chemokines. Stem Cells 26, 3139-3149.

Barrios-Rodiles, M., Brown, K.R., Ozdamar, B., Bose, R., Liu, Z., Donovan, R.S., Shinjo, F., Liu, Y., Dembowy, J., Taylor, I.W., Luga, V., Przulj, N., Robinson, M., Suzuki, H., Hayashizaki, Y., Jurisica, I., Wrana, J.L., 2005. High-throughput mapping of a dynamic signaling network in mammalian cells. Science 307, 1621-1625.

Baye, L.M., Link, B.A., 2007. Interkinetic nuclear migration and the selection of neurogenic cell divisions during vertebrate retinogenesis. J. Neurosci. 27, 10143-10152.

Becker, C.G., Becker, T., 2008. Adult zebrafish as a model for successful central nervous system regeneration. Restor. Neurol. Neurosci. 26, 71-80.

Bernardos, R.L., Barthel, L.K., Meyers, J.R., Raymond, P.A., 2007. Late-stage neuronal progenitors in the retina are radial Müller glia that function as retinal stem cells. J. Neurosci. 27, 7028-7040.
Bernardos, R.L., Lentz, S.I., Wolfe, M.S., Raymond, P.A., 2005. Notch-Delta signaling is required for spatial patterning and Müller glia differentiation in the zebrafish retina. Dev. Biol. 278, 381-395.

Bernardos, R.L., Raymond, P.A., 2006. GFAP transgenic zebrafish. Gene Exp. Patterns 6, 1007-1013.

Besser, M., Jagatheaswaran, M., Reinhard, J., Schaffelke, P., Faissner, A., 2012. Tenascin $C$ regulates proliferation and differentiation processes during embryonic retinogenesis and modulates the de-differentiation capacity of Müller glia by influencing growth factor responsiveness and the extracellular matrix compartment. Dev. Biol. 369, 163-176.

Bhattacharjee, J., Sanyal, S., 1975. Developmental origin and early differentiation of retinal Müller cells in mice. J. Anat. 120, 367-372.

Bibliowicz, J., Gross, J.M., 2009. Expanded progenitor populations, vitreo-retinal abnormalities, and Müller glial reactivity in the zebrafish leprechaun/ patched2 retina. BMC Dev. Biol. 9, 52.

Blaxter, J.H.S., 1975. The eyes of larval fish. In: Ali, M.A. (Ed.), Vision in Fishes: New Approaches to Research, Series A, Life Sciences ed. Plenum Press, New York, pp. $427-443$

Blaxter, J.H.S., Staines, M., 1970. Pure-cone retinae and retinomotor responses in larval teleosts. J. Mar. Biol. Assoc. U. K. 48, 17-28.

Bonni, A., Sun, Y., Nadal-Vicens, M., Bhatt, A., Frank, D.A., Rozovsky, I., Stahl, N., Yancopoulos, G.D., Greenberg, M.E., 1997. Regulation of gliogenesis in the central nervous system by the JAK-STAT signaling pathway. Science $278,477-$ 483.

Boucher, S.E., Hitchcock, P.F., 1998. Insulin-related growth factors stimulate proliferation of retinal progenitors in the goldfish. J. Comp. Neurol. 394, 386-394.

Braisted, J.E., Essman, T.F., Raymond, P.A., 1994. Selective regeneration of photoreceptors in goldfish retina. Development 120, 2409-2419.

Braisted, J.E., Raymond, P.A., 1992. Regeneration of dopaminergic neurons in goldfish retina. Development 114, 913-919.

Braisted, J.E., Raymond, P.A., 1993. Continued search for the cellular signals that regulate regeneration of dopaminergic neurons in goldfish retina. Dev. Brain Res. 76, 221-232.

Branchek, T., Bremiller, R., 1984. The development of photoreceptors in the zebrafish, Brachydanio rerio. I. Structure. J. Comp. Neurol. 224, 107-115.

Bringmann, A., Francke, M., Reichenbach, A., 2003. Müller Cells in Retinopathies. In Advances in Molecular and Cell Biology. Elsevier, pp. 1117-1132.

Bringmann, A., Iandiev, I., Pannicke, T., Wurm, A., Hollborn, M., Wiedemann, P., Osborne, N.N., Reichenbach, A., 2009. Cellular signaling and factors involved in Müller cell gliosis: neuroprotective and detrimental effects. Prog. Retin Eye Res. 28, 423-451.

Bringmann, A., Pannicke, T., Grosche, J., Francke, M., Wiedemann, P., Skatchkov, S.N., Osborne, N.N., Reichenbach, A., 2006. Müller cells in the healthy and diseased retina. Prog. Retin Eye Res. 25, 397-424.

Brockerhoff, S.E., Fadool, J.M., 2011. Genetics of photoreceptor degeneration and regeneration in zebrafish. Cell. Mol. Life Sci. CMLS 68, 651-659.

Brockes, J.P., Kumar, A., 2002. Plasticity and reprogramming of differentiated cells in amphibian regeneration. Nat. Rev. Mol. Cell Biol. 3, 566-574.

Brown, M.E., 1957. The Physiology of Fishes. Academic Press, New York.

Burke, J.M., Smith, J.M., 1981. Retinal proliferation in response to vitreous hemoglobin or iron. Investig. Ophthalmol. Vis. Sci. 20, 582-592.

Calinescu, A.A., Vihtelic, T.S., Hyde, D.R., Hitchcock, P.F., 2009. Cellular expression of midkine-a and midkine-b during retinal development and photoreceptor regeneration in zebrafish. J. Comp. Neurol. 514, 1-10.

Cameron, D.A., 2000. Cellular proliferation and neurogenesis in the injured retina of adult zebrafish. Vis. Neurosci. 17, 789-797.

Cameron, D.A., Carney, L.H., 2000. Cell mosaic patterns in the native and regenerated inner retina of zebrafish: implications for retinal assembly. J. Comp. Neurol. 416, 356-367.

Cameron, D.A., Carney, L.H., 2004. Cellular patterns in the inner retina of adult zebrafish: quantitative analyses and a computational model of their formation. J. Comp. Neurol. 471, 11-25.

Cameron, D.A., Cornwall, M.C., MacNichol, E.F., 1997. Visual pigment assignments in regenerated retina. J. Neurosci. 17, 917-923.

Cameron, D.A., Easter, S.S., 1995. Cone photoreceptor regeneration in adult fish retina: phenotypic determination and mosaic pattern formation. Vis. Neurosci. $15,2255-2271$.

Cameron, D.A., Gentile, K.L., Middleton, F.A., Yurco, P., 2005. Gene expression profiles of intact and regenerating zebrafish retina. Mol. Vis. 11, 775-791.

Campbell, K., Gotz, M., 2002. Radial glia: multi-purpose cells for vertebrate brain development. Trends Neurosci. 25, 235-238.

Carl, M., Loosli, F., Wittbrodt, J., 2002. Six3 inactivation reveals its essential role for the formation and patterning of the vertebrate eye. Development 129, 40574063.

Carter-Dawson, L.D., LaVail, M.M., 1979. Rods and cones in the mouse retina. II. Autoradiographic analysis of cell generation using tritiated thymidine. J. Comp. Neurol. 188, 263-272.

Centanin, L., Hoeckendorf, B., Wittbrodt, J., 2011. Fate restriction and multipotency in retinal stem cells. Cell Stem Cell 9,553-562.

Cerveny, K.L., Cavodeassi, F., Turner, K.J., de Jong-Curtain, T.A., Heath, J.K., Wilson, S.W., 2010. The zebrafish flotte lotte mutant reveals that the local retinal environment promotes the differentiation of proliferating precursors emerging from their stem cell niche. Development 137, 2107-2115.

Cerveny, K.L., Varga, M., Wilson, S.W., 2012. Continued growth and circuit building in the anamniote visual system. Dev. Neurobiol. 72, 328-345. 
Chablais, F., Jaźwińska, A., 2012. The regenerative capacity of the zebrafish heart is dependent on TGFbeta signaling. Development 139, 1921-1930.

Chapouton, P., Jagasia, R., Bally-Cuif, L., 2007. Adult neurogenesis in nonmammalian vertebrates. Bioessays 29, 745-757.

Chapouton, P., Skupien, P., Hesl, B., Coolen, M., Moore, J.C., Madelaine, R. Kremmer, E., Faus-Kessler, T., Blader, P., Lawson, N.D., Bally-Cuif, L., 2010. Notch activity levels control the balance between quiescence and recruitment of adult neural stem cells. J. Neurosci. 30, 7961-7974.

Chinen, A., Hamaoka, T., Yamada, Y., Kawamura, S., 2003. Gene duplication and spectral diversification of cone visual pigments of zebrafish. Genetics 163, 663675.

Chiu, J.F., Mack, A.F., Fernald, R.D., 1995. Daily rhythm of cell proliferation in the teleost retina. Brain Res, 673, 119-125.

Choi, W.Y., Poss, K.D., 2012. Cardiac regeneration. Curr. Top. Dev. Biol. 100, 319-344.

Chow, R.L., Lang, R.A., 2001. Early eye development in vertebrates. Annu. Rev. Cell Dev. Biol. 17, 255-296.

Chuang, J.C., Raymond, P.A., 2002. Embryonic origin of the eyes in teleost fish. Bioessays 24, 519-529.

Close, J.L., Gumuscu, B., Reh, T.A., 2005. Retinal neurons regulate proliferation of postnatal progenitors and Müller glia in the rat retina via TGF beta signaling. Development 132, 3015-3026.

Close, J.L., Liu, J., Gumuscu, B., Reh, T.A., 2006. Epidermal growth factor receptor expression regulates proliferation in the postnatal rat retina. Glia 54, 94-104.

Craig, S.E., Thummel, R., Ahmed, H., Vasta, G.R., Hyde, D., Hitchcock, P.F., 2010. The zebrafish galectin Drgal1-L2 is expressed by proliferating Müller glia and photoreceptor progenitors and regulates the regeneration of rod photoreceptors. Investig. Ophthalmol. Vis. Sci. 51, 3244-3252.

Craig, S.E.L., Calinescu, A.-A., Hitchcock, P., 2008. Identification of the molecular signatures integral to regenerating photoreceptors in the retina of the zebrafish J. Ocul. Biol. Dis. Inf. 1, 73-84.

Das, A.V., Mallya, K.B., Zhao, X., Ahmad, F., Bhattacharya, S., Thoreson, W.B., Hegde, G.V., Ahmad, I., 2006. Neural stem cell properties of Müller glia in the mammalian retina: regulation by Notch and Wnt signaling. Dev. Biol. 299, $283-$ 302.

Das, T., Payer, B., Cayouette, M., Harris, W.A., 2003. In vivo time-lapse imaging of cel divisions during neurogenesis in the developing zebrafish retina. Neuron 37 597-609.

Del Bene, F., Tessmar-Raible, K., Wittbrodt, J., 2004. Direct interaction of geminin and Six3 in eye development. Nature 427, 745-749.

Del Bene, F., Wehman, A.M., Link, B.A., Baier, H., 2008. Regulation of neurogenesis by interkinetic nuclear migration through an apical-basal notch gradient. Cell 134 1055-1065.

Del Debbio, C.B., Balasubramanian, S., Parameswaran, S., Chaudhuri, A., Qiu, F. Ahmad, I., 2010. Notch and Wnt signaling mediated rod photoreceptor regeneration by Müller cells in adult mammalian retina. PLoS One 5, e12425.

Doetsch, F., 2003. A niche for adult neural stem cells. Curr. Opin. Genet. Dev. 13, $543-550$.

Dong, Z., Yang, N., Yeo, S.Y., Chitnis, A., Guo, S., 2012. Intralineage directional Notch signaling regulates self-renewal and differentiation of asymmetrically dividing radial glia. Neuron $74,65-78$.

Dyer, M.A., Cepko, C.L., 2000a. Control of Müller glial cell proliferation and activation following retinal injury. Nat. Neurosci. 3, 873-880.

Dyer, M.A., Cepko, C.L., 2000b. p57(Kip2) regulates progenitor cell proliferation and amacrine interneuron development in the mouse retina. Development 127 3593-3605.

Dyer, M.A., Cepko, C.L., 2001. p27Kip1 and p57Kip2 regulate proliferation in distinct retinal progenitor cell populations. J. Neurosci. 21, 4259-4271.

Easter, S.S., 1983. Postnatal neurogenesis and changing connections. Trends Neurosci. $6,53-56$

Easter Jr., S.S., Malicki, J.J., 2002. The zebrafish eye: developmental and genetic analysis. Results Probl. Cell. Differ. 40, 346-370.

Eiraku, M., Takata, N., Ishibashi, H., Kawada, M., Sakakura, E., Okuda, S., Sekiguchi, K., Adachi, T., Sasai, Y., 2011. Self-organizing optic-cup morphogenesis in threedimensional culture. Nature 472, 51-56.

El Yakoubi, W., Borday, C., Hamdache, J., Parain, K., Tran, H.T., Vleminckx, K. Perron, M., Locker, M., 2012. Hes4 controls proliferative properties of neural stem cells during retinal ontogenesis. Stem Cells 30, 2784-2795.

Engström, K., 1963. Cone types and cone arrangements in teleost retinae. Acta Zool 44, 179-243.

Fadool, J.M., Dowling, J.E., 2008. Zebrafish: a model system for the study of eye genetics. Prog. Retin Eye Res. 27, 89-110.

Faillace, M.P., Julian, D., Korenbrot, J.I., 2002. Mitotic activation of proliferative cells in the inner nuclear layer of the mature fish retina: regulatory signals and molecular markers. J. Comp. Neurol. 451, 127-141.

Faissner, A., Kruse, J., 1990. J1/Tenascin is a repulsive substrate for central nervous system neurons. Neuron 5 (627), 637.

Fausett, B.V., Goldman, D., 2006. A role for alpha1 tubulin-expressing Müller glia in regeneration of the injured zebrafish retina. J. Neurosci. 26, 6303 6313.

Fausett, B.V., Gumerson, J.D., Goldman, D., 2008. The proneural basic helix-loophelix gene ascl1a is required for retina regeneration. J. Neurosci. 28, 1109-1117. Fernald, R.D., 1991. Teleost vision: seeing while growing. J. Exp. Zool. 5, 167-180.

Fimbel, S.M., Montgomery, J.E., Burket, C.T., Hyde, D.R., 2007. Regeneration of inne retinal neurons after intravitreal injection of ouabain in zebrafish. J. Neurosci. $27,1712-1724$
Fischer, A.J., Bongini, R., 2010. Turning Müller glia into neural progenitors in the retina. Mol. Neurobiol. 42, 199-209.

Fischer, A.J., McGuire, C.R., Dierks, B.D., Reh, T.A., 2002. Insulin and fibroblast growth factor 2 activate a neurogenic program in Müller glia of the chicken retina. J. Neurosci. 22, 9387-9398.

Fischer, A.J., Reh, T.A., 2003. Potential of Müller glia to become neurogenic retinal progenitor cells. Glia 43, 70-76.

Fishell, G., Kriegstein, A.R., 2003. Neurons from radial glia: the consequences of asymmetric inheritance. Curr. Opin. Neurobiol. 13, 34-41.

Fisher, S.K., Lewis, G.P., 2003. Müller cell and neuronal remodeling in retinal detachment and reattachment and their potential consequences for visual recovery: a review and reconsideration of recent data. Vis. Res. 43, 887897.

Forbes-Osborne, M.A., Wilson, S.G., Morris, A.C., 2013. Insulinoma-associated 1a (Insm1a) is required for photoreceptor differentiation in the zebrafish retina. Dev. Biol. 380, 157-171.

Fraser, B., DuVal, M.G., Wang, H., Allison, W.T., 2013. Regeneration of cone photoreceptors when cell ablation is primarily restricted to a particular cone subtype. PLoS One 8, e55410.

Fuentealba, L.C., Obernier, K., Alvarez-Buylla, A., 2012. Adult neural stem cells bridge their niche. Cell Stem Cell 10, 698-708.

Fujita, S., Hori, M., 1963. Analysis of cytogenesis in chick retina by ${ }^{3} \mathrm{H}$-thymidine autoradiography. Arch. Histol. Cytol. 23, 359-366.

Furukawa, T., Mukherjee, S., Bao, Z.Z., Morrow, E.M., Cepko, C.L., 2000. rax, Hes1, and notch1 promote the formation of Müller glia by postnatal retinal progenitor cells. Neuron 26, 383-394.

Gallina, D., Todd, L., Fischer, A.J., 2013. A comparative analysis of Müller gliamediated regeneration in the vertebrate retina. Exp. Eye Res. (in press).

Gascon, E., Vutskits, L., Kiss, J.Z., 2010. The Role of PSA-NCAM in adult neurogenesis. Adv, Exp. Med. Biol. 663, 127-136.

Gemberling, M., Bailey, TJ. Hyde, D.R., Poss, K.D., 2013. The zebrafish as a model for complex tissue regeneration. Trends Gen. 29, 611-620.

Gnana-Prakasam, J.P., Martin, P.M., Smith, S.B., Ganapathy, V., 2010. Expression and function of iron-regulatory proteins in retina. IUBMB Life 62, 363-370.

Goldsmith, P., Harris, W.A., 2003. The zebrafish as a tool for understanding the biology of visual disorders. Semin. Cell Dev. Biol. 14, 11-18.

Gonzalez-Nunez, V., Nocco, V., Budd, A., 2010. Characterization of drCol 15a1b: a novel component of the stem cell niche in the zebrafish retina. Stem Cells 28 1399-1411.

Gorsuch, R.A., Hyde, D., 2013. Regulation of Müller glial dependent neuronal regeneration in the damaged adult zebrafish retina. Exp. Eye Res. (in press).

Gosens, I., den Hollander, A.I., Cremers, F.P., Roepman, R., 2008. Composition and function of the Crumbs protein complex in the mammalian retina. Exp. Eye Res. $86,713-726$.

Gotz, M., Huttner, W.B., 2005. The cell biology of neurogenesis. Nat. Rev. Mol. Cell Biol. 6, 777-788.

Grandbarbe, L., Bouissac, J., Rand, M., Hrabe de Angelis, M., ArtavanisTsakonas, S., Mohier, E., 2003. Delta-Notch signaling controls the generation of neurons/glia from neural stem cells in a stepwise process. Development 130, 1391-1402.

Grandel, H., Brand, M., 2013. Comparative aspects of adult neural stem cell activity in vertebrates. Dev. Genes Evol. 223, 131-147.

Grandel, H., Kaslin, J., Ganz, J., Wenzel, I., Brand, M., 2006. Neural stem cells and neurogenesis in the adult zebrafish brain: origin, proliferation dynamics, migration and cell fate. Dev. Biol. 295, 263-277.

Habib, S.J., Chen, B.C., Tsai, F.C., Anastassiadis, K., Meyer, T., Betzig, E., Nusse, R., 2013. A localized Wnt signal orients asymmetric stem cell division in vitro. Science 339, 1445-1448.

Hafler, B.P., Surzenko, N., Beier, K.T., Punzo, C., Trimarchi, J.M., Kong, J.H., Cepko, C.L., 2012. Transcription factor Olig2 defines subpopulations of retinal progenitor cells biased toward specific cell fates. Proc. Natl. Acad. Sci. U. S. A. 109, 7882 7887.

Hagedorn, M., Fernald, R., 1992. Retinal growth and cell addition during embryogenesis in the teleost, Haplochromis burtoni. J. Comp. Neurol. 321, 193-208.

Halder, G., Johnson, R.L., 2011. Hippo signaling: growth control and beyond. Development 138, 9-22.

Harris, W.A., Perron, M., 1998. Molecular recapitulation: the growth of the vertebrate retina. Int. J. Dev. Biol. 42, 299-304.

Hartfuss, E., Galli, R., Heins, N., Gotz, M., 2001. Characterization of CNS precursor subtypes and radial glia. Dev. Biol. 229, 15-30.

He, J., Zhang, G., Almeida, A.D., Cayouette, M., Simons, B.D., Harris, W.A., 2012. How variable clones build an invariant retina Neuron 75, 786-798.

Hieber, V., Dai, X., Foreman, M., Goldman, D., 1998. Induction of alpha1-tubulin gene expression during development and regeneration of the fish central nervous system. J. Neurobiol. 37, 429-440.

Hinds, J. 1968. Autoradiographic study of histogenesis in the mouse olfactory bulb. II. Cell proliferation and migration. J. Comp. Neurol. 134 (305), 322.

Hinds, J.W., Hinds, P.L., 1974. Early ganglion cell differentiation in the mouse retina: an electron microscopic analysis utlizing serial sections. Dev. Biol. 37, 381-416.

Hinds, J.W., Hinds, P.L., 1978. Early development of amacrine cells in the mouse retina: an electron microscopic, serial section analysis. J. Comp. Neurol. 179, $277-300$.

Hisatomi, O., Satoh, T., Barthel, L.K., Stenkamp, D.L., Raymond, P.A., Tokunaga, F, 1996. Molecular cloning and characterization of the putative ultravioletsensitive visual pigment of goldfish. Vis. Res. 36, 933-939. 
Hitchcock, P., Ochocinska, M., Sieh, A., Otteson, D., 2004. Persistent and injuryinduced neurogenesis in the vertebrate retina. Prog. Retin Eye Res. 23, 183-194.

Hitchcock, P.F., 1997. Tracer coupling among regenerated amacrine cells in the retina of the goldfish. Vis. Neurosci. 14, 463-472.

Hitchcock, P.F., Cirenza, P., 1994. Synaptic organization of regenerated retina in the goldfish. J. Comp. Neurol. 343, 609-616.

Hitchcock, P.F., Lindsey Myhr, K.J., Easter, S.S., Mangione-Smith, R., Jones, D.D., 1992. Local regeneration in the retina of the goldfish. J. Neurobiol. 23, 187-203.

Hitchcock, P.F., Macdonald, R.E., VanDeRyt, J.T., Wilson, S.W., 1996. Antibodies against pax6 immunostain amacrine and ganglion cells and neuronal progenitors, but not rod precursors, in the normal and regenerating retina of the goldfish. J. Neurobiol. 29, 399-413.

Hitchcock, P.F., Raymond, P.A., 1992. Retinal regeneration. Trends Neurosci. 15, 103108.

Hitchcock, P.F., Raymond, P.A., 2004. The teleost retina as a model for developmental and regeneration biology. Zebrafish 1, 257-271.

Hitchcock, P.F., Vanderyt, J.T., 1994. Regeneration of the dopamine-cell mosaic in the retina of the goldfish. Vis. Neurosci. 11, 209-217.

Hochedlinger, K., Plath, K., 2009. Epigenetic reprogramming and induced pluripotency. Development 136, 509-523.

Hochmann, S., Kaslin, J., Hans, S., Weber, A., Machate, A., Geffarth, M., Funk, R.H., Brand, M., 2012. Fgf signaling is required for photoreceptor maintenance in the adult zebrafish retina. PLoS One 7, e30365.

Hollborn, M., Tenckhoff, S., Jahn, K., Iandiev, I., Biedermann, B., Schnurrbusch, U.E. Limb, G.A., Reichenbach, A., Wolf, S., Wiedemann, P., Kohen, L., Bringmann, A., 2005. Changes in retinal gene expression in proliferative vitreoretinopathy: glial cell expression of HB-EGF. Mol. Vis. 11, 397-413.

Hollyfield, J., 1972. Histogenesis of the retinal of the killifish Fundulus heteroclitus. J. Comp. Neurol. 144 (373), 380.

Hollyfield, J.G., 1968. Differential addition of cells to the retina in Rana pipiens tadpoles. Dev. Biol. 18, 163-179.

Hollyfield, J.G., 1971. Differential growth of the neural retina in Xenopus laevis larvae. Dev. Biol. 24, 264-286.

Holt, C.E., Bertsch, T.W., Ellis, H.M., Harris, W.A., 1988. Cellular determination in the Xenopus retina is independent of lineage and birth date. Neuron 1, 15-26.

Hsieh, J., 2012. Orchestrating transcriptional control of adult neurogenesis. Genes. Dev. 26, 1010-1021.

Hu, M., Easter, S.S., 1999. Retinal neurogenesis: the formation of the initial central patch of postmitotic cells. Dev. Biol. 207, 309-321.

Huttner, W.B., Brand, M., 1997. Asymmetric division and polarity of neuroepithelial cells, Curr. Opin. Neurobiol. 7, 29-39.

Ichida, J.K., Blanchard, J., Lam, K., Son, E.Y., Chung, J.E., Egli, D., Loh, K.M., Carter, A.C., Di Giorgio, F.P., Koszka, K., Huangfu, D., Akutsu, H., Liu, D.R., Rubin, L.L., Eggan, K., 2009. A small-molecule inhibitor of tgf-Beta signaling replaces sox2 in reprogramming by inducing nanog. Cell Stem Cell 5, 491-503.

Ikeda, H., Osakada, F., Watanabe, K., Mizuseki, K., Haraguchi, T., Miyoshi, H., Kamiya, D., Honda, Y., Sasai, N., Yoshimura, N., Takahashi, M., Sasai, Y., 2005. Generation of $\mathrm{Rx}+$ /Pax6+ neural retinal precursors from embryonic stem cells. Proc. Natl. Acad. Sci. U. S. A. 102, 11331-11336.

Ikeda, T., Puro, D.G., 1994. Nerve growth factor: a mitogenic signal for retinal Müieller glial cells. Brain Res. 649, 260-264.

Ikeda, T., Puro, D.G., 1995. Regulation of retinal glial cell proliferation by antiproliferative molecules. Exp. Eye Res. 60, 435-443.

Inbal, A., Kim, S.H., Shin, J., Solnica-Krezel, L., 2007. Six3 represses nodal activity to establish early brain asymmetry in zebrafish. Neuron 55, 407-415.

Jadhav, A.P., Cho, S.H., Cepko, C.L., 2006a. Notch activity permits retinal cells to progress through multiple progenitor states and acquire a stem cell property. Proc. Natl. Acad. Sci. U. S. A. 103, 18998-19003.

Jadhav, A.P., Mason, H.A., Cepko, C.L., 2006b. Notch 1 inhibits photoreceptor production in the developing mammalian retina. Development 133, 913-923.

Jadhav, A.P., Roesch, K., Cepko, C.L., 2009. Development and neurogenic potential of Müller glial cells in the vertebrate retina. Prog. Retin Eye Res. 28, 249-262.

Jannie, K.M., Stipp, C.S., Weiner, J.A., 2012. ALCAM regulates motility, invasiveness, and adherens junction formation in uveal melanoma cells. PLoS One 7, e39330.

Johns, P.R., 1977. Growth of the adult goldfish eye. III. Source of the new retinal cells. J. Comp. Neurol. 176, 343-358.

Johns, P.R., 1981. Growth of fish retinas. Am. Zool. 21, 447-458.

Johns, P.R., 1982. The formation of photoreceptors in larval and adult goldfish. J. Neurosci. 2, 179-198.

Johns, P.R., Easter, S.S., 1977. Growth of the adult goldfish eye II. Increase in retinal cell number. J. Comp. Neurol. 176, 331-341.

Johns, P.R., Fernald, R.D., 1981. Genesis of rods in teleost fish retina. Nature 293, $141-142$.

Joly, S., Pernet, V., Samardzija, M., Grimm, C., 2011. Pax6-positive Müller glia cells express cell cycle markers but do not proliferate after photoreceptor injury in the mouse retina. Glia 59, 1033-1046.

Jopling, C., Boue, S., Izpisua Belmonte, J.C., 2011. Dedifferentiation, transdifferentiation and reprogramming: three routes to regeneration. Nat. Rev. Mol. Cell Biol. 12, 79-89.

Julian, D., Ennis, K., Korenbrot, J.I., 1998. Birth and fate of proliferative cells in the inner nuclear layer of the mature fish retina. J. Comp. Neurol. 394, 271-282.

Jusuf, P., Harris, W.A., Poggi, L., 2013. Imaging retinal progenitor lineages in developing zebrafish embryos. Cold Spring Harb. Protoc. 2013.

Kahn, A.J., 1974. An autoradiographic analysis of the time to appearance of neurons in the developing chick retina. Dev. Biol. 38, 30-40.
Kamakura, S., Oishi, K., Yoshimatsu, T., Nakafuku, M., Masuyama, N., Gotoh, Y., 2004 Hes binding to STAT3 mediates crosstalk between Notch and JAK-STAT signalling. Nat. Cell Biol. 6, 547-554.

Karl, M.O., Hayes, S., Nelson, B.R., Tan, K., Buckingham, B., Reh, T.A., 2008. Stimulation of neural regeneration in the mouse retina. Proc. Natl. Acad. Sci. U. S. A. 105, 19508-19513.

Karl, M.O., Reh, T.A., 2010. Regenerative medicine for retinal diseases: activating endogenous repair mechanisms. Trends Mol. Med. 16, 193-202.

Kassen, S.C., Ramanan, V., Montgomery, J.E., Burket, C.T., Liu, C.G., Vihtelic, T.S. Hyde, D.R., 2007. Time course analysis of gene expression during light-induced photoreceptor cell death and regeneration in albino zebrafish. Dev. Neurobiol. 67, 1009-1031.

Kassen, S.C., Thummel, R., Campochiaro, L.A., Harding, M.J., Bennett, N.A., Hyde, D.R. 2009. CNTF induces photoreceptor neuroprotection and Müller glial cell proliferation through two different signaling pathways in the adult zebrafish retina. Exp. Eye Res. 88, 1051-1064.

Kästner, R., Wolburg, H., 1982. Functional regeneration of the visual system in teleosts. Comparative investigations after optic nerve crush and damage of the retina. Z. Naturforsch. 37, 1274-1280.

Katakura, M., Hashimoto, M., Shahdat, H.M., Gamoh, S., Okui, T., Matsuzaki, K. Shido, O., 2009. Docosahexaenoic acid promotes neuronal differentiation by regulating basic helix-loop-helix transcription factors and cell cycle in neural stem cells. Neuroscience 160, 651-660.

Kechad, A., Jolicoeur, C., Tufford, A., Mattar, P., Chow, R.W., Harris, W.A. Cayouette, M., 2012. Numb is required for the production of terminal asymmetric cell divisions in the developing mouse retina. J. Neurosci. 32, 1719717210.

Kelley, M.W., Turner, J.K., Reh, T.A., 1994. Retinoic acid promotes differentiation of photoreceptors in vitro. Development 120, 2091-2102.

Kim, N.G., Koh, E., Chen, X., Gumbiner, B.M., 2011. E-cadherin mediates contact inhibition of proliferation through Hippo signaling-pathway components. Proc. Natl. Acad. Sci. U. S. A. 108, 11930-11935.

Kizil, C., Kaslin, J., Kroehne, V., Brand, M., 2012. Adult neurogenesis and brain regeneration in zebrafish. Dev. Neurobiol. 72, 429-461.

Kljavin, I.J., 1987. Early development of photoreceptors in the ventral retina of the zebrafish embryo. J. Comp. Neurol. 260, 461-471.

Knapp, D., Tanaka, E.M., 2012. Regeneration and reprogramming. Curr. Opin. Genet. Dev. 22, 485-493.

Korzh, V., Sleptsova, I., Liao, J., He, J., Gong, Z., 1998. Expression of zebrafish bHLH genes ngn1 and nrd defines distinct stages of neural differentiation. Dev. Dyn. 213, 92-104.

Kriegstein, A., Alvarez-Buylla, A., 2009. The glial nature of embryonic and adult neural stem cells. Annu. Rev. Neurosci. 32, 149-184.

Kroehne, V., Freudenreich, D., Hans, S., Kaslin, J., Brand, M., 2011. Regeneration of the adult zebrafish brain from neurogenic radial glia-type progenitors. Development 138, 4831-4841.

Kubo, F., Nakagawa, S., 2009. Hairy1 acts as a node downstream of Wnt signaling to maintain retinal stem cell-like progenitor cells in the chick ciliary marginal zone. Development 136, 1823-1833.

Kubo, F., Takeichi, M., Nakagawa, S., 2003. Wnt2b controls retinal cell differentiation at the ciliary marginal zone. Development 130, 587-598.

Kubo, F., Takeichi, M., Nakagawa, S., 2005. Wnt2b inhibits differentiation of retinal progenitor cells in the absence of Notch activity by downregulating the expression of proneural genes. Development 132, 2759-2770.

Kubota, R., Hokoc, J.N., Moshiri, A., McGuire, C., Reh, T.A., 2002. A comparative study of neurogenesis in the retinal ciliary marginal zone of homeothermic vertebrates. Brain Res. Dev. Brain Res. 134, 31-41.

Kunz, Y.W., Ennis, S., Wise, C., 1983. Ontogeny of the photoreceptors in the embryonic retina of the viviparous guppy, Poecilia reticulatus P. (Teleostei). An electron- microscopical study. Cell Tissue Res. 230, 469-486.

Kurz-Isler, G., Wolburg, H., 1982. Morphological study on the regeneration of the retina in the rainbow trout after ouabain-induced damage: evidence for dedifferentiation of photoreceptors. Cell Tissue Res. 225, 165178.

Kustermann, S., Hildebrandt, H., Bolz, S., Dengler, K., Kohler, K., 2010. Genesis of rods in the zebrafish retina occurs in a microenvironment provided by polysialic acid-expressing Müller glia. J. Comp. Neurol. 518, 636-646.

Kuwabara, T., Weidman, T.A., 1974. Development of the prenatal rat retina. Investig. Ophthalmol. Vis. Sci. 13, 725-739.

Kyritsis, N., Kizil, C., Zocher, S., Kroehne, V., Kaslin, J., Freudenreich, D., Iltzsche, A. Brand, M., 2012. Acute inflammation initiates the regenerative response in the adult zebrafish brain. Science 338, 1353-1356.

La Torre, A., Georgi, S., Reh, T.A., 2013. Conserved microRNA pathway regulates developmental timing of retinal neurogenesis. Proc. Natl. Acad. Sci. U. S. A. 110, E2362-E2370.

Lamba, D.A., Gust, J., Reh, T.A., 2009a. Transplantation of human embryonic stem cell-derived photoreceptors restores some visual function in Crx-deficient mice. Cell Stem Cell 4, 73-79.

Lamba, D.A., Karl, M.O., Reh, T.A., 2009b. Strategies for retinal repair: cell replacement and regeneration. Prog. Brain Res. 175, 23-31.

Lamba, D.A., Karl, M.O., Ware, C.B., Reh, T.A., 2006. Efficient generation of retinal progenitor cells from human embryonic stem cells. Proc. Natl. Acad. Sci. U. S. A 103, 12769-12774.

LaVail, M.M., Rapaport, D.H., Rakic, P., 1991. Cytogenesis in the monkey retina. J. Comp. Neurol. 309, 86-114. 
Lawrence, J.M., Singhal, S., Bhatia, B., Keegan, D.J., Reh, T.A., Luthert, P.J., Khaw, P.T., Limb, G.A., 2007. MIO-M1 cells and similar Müller glial cell lines derived from adult human retina exhibit neural stem cell characteristics. Stem Cells 25 2033-2043.

Layer, P.G., Willbold, E., 1993. Histogenesis of the avian retina in reaggregation culture: from dissociated cells to laminar neuronal networks. Int. Rev. Cytol. $146,1-47$.

Lee, Y., Grill, S., Sanchez, A., Murphy-Ryan, M., Poss, K.D., 2005. Fgf signaling instructs position-dependent growth rate during zebrafish fin regeneration. Development 132, 5173-5183.

Lemmon, V., Rieser, G., 1983. The developmental distribution of vimentin in the chick retina. Brain Res. 313, 191-197.

Lenkowski, J.R., Qin, Z., Sifuentes, C.J., Thummel, R., Soto, C.M., Moens, C.B., Raymond, P.A., 2013. Retinal regeneration in adult zebrafish requires regulation of TGFb signaling. Glia 61, 1687-1697.

Levine, E.M., Close, J., Fero, M., Ostrovsky, A., Reh, T.A., 2000. p27(Kip1) regulates cell cycle withdrawal of late multipotent progenitor cells in the mammalian retina. Dev. Biol. 219, 299-314.

Lillien, L., Cepko, C., 1992. Control of proliferation in the retina: temporal changes in responsiveness to FGF and TGF alpha. Development 115, 253-266.

Lindsey, A.E., Wall, R.M., Powers, M.K., 1995. Recovery of visual behavior in adult goldfish with regenerating retina. Investig. Ophthalmol. Vis. Sci. Suppl. 36, 60.

Link, B.A., Darland, T. 2001. Genetic analysis of initial and ongoing retinogenesis in the zebrafish: comparing the central neuroepithelium and marginal zone. Prog. Brain Res. 131, 565-577.

Liu, B., Hunter, D.J., Rooker, S., Chan, A., Paulus, Y.M., Leucht, P., Nusse, Y., Nomoto, H. Helms, J.A., 2013. Wnt signaling promotes Müller cell proliferation and survival after injury. Investig. Ophthalmol. Vis. Sci. 54, 444-453.

Liu, Q., Londraville, R.L., Azodi, E., Babb, S.G., Chiappini-Williamson, C., Marrs, J.A., Raymond, P.A., 2002. Up-regulation of cadherin-2 and cadherin-4 in regenerating visual structures of adult zebrafish. Exp. Neurol. 177, 396-406.

Locket, N.A., 1980. Variation of architecture with size in the multiple-bank retina of a deep-sea teleost, Chauliodus sloani. Proc. R. Soc. Lond. B Biol. Sci. 208, 223-242.

Lombardo, F., 1968. La rigenerazione della retina negli adulti di un Teleosteo (Regeneration of the retina in an adult teleost). Accad. Lincei-Rend. Sci. Fis. Mat. Nat. Ser. 8 (45), 631-635.

Lombardo, F., 1972. Andamento e localizzazione della mitosi durante la rigenerazione della retina di un Teleosteo adulto (Time course and localization of mitoses during regeneration of the retina in an adult teleost). Accad. LinceiRend. Sci. Fis. Mat. Nat. Ser. 8 (53), 323-327.

Loosli, F., Koster, R.W. Carl, M., Krone, A., Wittbrodt, J., 1998. Six3, a medaka homologue of the Drosophila homeobox gene sine oculis is expressed in the anterior embryonic shield and the developing eye. Mech. Dev. 74, 159-164.

Loosli, F., Winkler, S., Wittbrodt, J., 1999. Six3 overexpression initiates the formation of ectopic retina. Genes. Dev. 13, 649-654.

Luo, J., Uribe, R.A., Hayton, S., Calinescu, A.A., Gross, J.M., Hitchcock, P.F., 2012 Midkine-A functions upstream of Id2a to regulate cell cycle kinetics in the developing vertebrate retina. Neural Dev. 7, 33.

Lyall, A.H., 1957. The growth of the trout retina. Q. J. Microsc. Sci. 98, 101-110.

Macdonald, R., Wilson, S.W., 1997. Distribution of Pax6 protein during eye development suggests discrete roles in proliferative and differentiated visual cells Roux's Arch. Dev. Biol. 206, 363-369.

Mack, A.F., Fernald, R.D., 1993. Regulation of cell division and rod differentiation in the teleost retina. Dev. Brain Res. 76, 183-187.

Mader, M.M., Cameron, D.A., 2004. Photoreceptor differentiation during retinal development, growth, and regeneration in a metamorphic vertebrate. J. Neurosci. 24, 11463-11472.

Maier, W., Wolburg, H., 1979. Regeneration of the goldfish retina after exposure to different doses of ouabain. Cell Tissue Res. 202, 99-118.

Makino, S., Whitehead, G.G., Lien, C.L., Kim, S., Jhawar, P., Kono, A., Kawata, Y., Keating, M.T., 2005. Heat-shock protein 60 is required for blastema formation and maintenance during regeneration. Proc. Natl. Acad. Sci. U. S. A. 102,14599-14604.

Mann, I.C., 1928. The process of differentiation of the retinal layers in vertebrates. Br. J. Ophthal. 12, 449-478.

Marc, R.E., Sperling, H.G., 1976. The chromatic organization of the goldfish cone mosaic. Vis. Res. 16, 1211-1224.

Marcus, R.C., Delaney, C.L., Easter Jr., S.S., 1999. Neurogenesis in the visual system of embryonic and adult zebrafish (Danio rerio). Vis. Neurosci. 16, 417-424.

Mensinger, A., Powers, M., 1999. Visual function in regenerating teleost retina following cytotoxic lesioning. Vis. Neurosci. 16, 241-251.

Merkle, F.T., Alvarez-Buylla, A., 2006. Neural stem cells in mammalian development. Curr. Opin. Cell Biol. 18, 704-709.

Meyer, R., 1978. Evidence from thymidine labeling for continuing growth of retina and tectum in juvenile goldfish. Exp. Neurol. 59, 99-111.

Meyers, J.R., Hu, L., Moses, A., Kaboli, K., Papandrea, A., Raymond, P.A., 2012. betaCatenin/Wnt signaling controls progenitor fate in the developing and regenerating zebrafish retina. Neural Dev. 7, 30.

Miller, F.D., Gauthier, A.S., 2007. Timing is everything: making neurons versus glia in the developing cortex. Neuron 54, 357-369.

Montgomery, J.E., Parsons, M.J., Hyde, D.R., 2010. A novel model of retinal ablation demonstrates that the extent of rod cell death regulates the origin of the regenerated zebrafish rod photoreceptors. J. Comp. Neurol. 518, 800-814.

Mori, T., Buffo, A., Gotz, M., 2005. The novel roles of glial cells revisited: the contribution of radial glia and astrocytes to neurogenesis. Curr. Top. Dev. Biol. 69, 67-99.
Morrens, J., Van Den Broeck, W., Kempermann, G., 2012. Glial cells in adult neurogenesis, Glia 60, 159-174.

Morris, A.C., Forbes-Osborne, M.A., Pillai, L.S., Fadool, J.M., 2011. Microarray analysis of XOPS-mCFP zebrafish retina identifies genes associated with rod photoreceptor degeneration and regeneration. Investig. Ophthalmol. Vis. Sci. 52, 2255-2266.

Morris, A.C., Scholz, T.L., Brockerhoff, S.E., Fadool, J.M., 2008. Genetic dissection reveals two separate pathways for rod and cone regeneration in the teleost retina. Dev. Neurobiol. 68, 605-619.

Morris, A.C., Schroeter, E.H., Bilotta, J., Wong, R.O., Fadool, J.M., 2005. Cone survival despite rod degeneration in XOPS-MCFP transgenic zebrafish. Investig. Ophthalmol. Vis. Sci. 46, 4762-4771.

Moshiri, A., Close, J., Reh, T.A., 2004. Retinal stem cells and regeneration. Int. J. Dev. Biol. 48, 1003-1014.

Moshiri, A., McGuire, C.R., Reh, T.A., 2005. Sonic hedgehog regulates proliferation of the retinal ciliary marginal zone in posthatch chicks. Dev. Dyn. 233, 66-75.

Moshiri, A., Reh, T.A., 2004. Persistent progenitors at the retinal margin of ptc $+/-$ mice. J. Neurosci. 24, 229-237.

Müller, H., 1952. Bau und Wachstum der Netzhaut des Guppy (Lebistes reticulatus) (Growth and development of the guppy retina (Lebistes reticulatus)). Zool. Jb 63, 275-324.

Murciano, A., Zamora, J., Lopez-Sanchez, J., Frade, J.M., 2002. Interkinetic nuclear movement may provide spatial clues to the regulation of neurogenesis. Mol. Cell. Neurosci. 21, 285-300.

Nagashima, M., Barthel, L.K., Raymond, P.A., 2013. Müller glia in injured zebrafish retinas divide asymmetrically once to generate neuronal progenitors that require $\mathrm{N}$-cadherin-mediated cell-cell adhesion to regenerate retinal neurons. Development 140, 4510-4521.

Nassar, K., Luke, J., Luke, M., Kamal, M., Abd El-Nabi, E., Soliman, M., Rohrbach, M., Grisanti, S., 2011. The novel use of decorin in prevention of the development of proliferative vitreoretinopathy (PVR). Graefes Arch. Clin. Exp. Ophthalmol. 249, 1649-1660.

Negishi, K., Shinagawa, S., 1993. Fibroblast growth factor induces proliferating cell nuclear antigen-immunoreactive cells in goldfish retina. Neurosci. Res. 18, 143156.

Negishi, K., Stell, W.K., Takasaki, Y., 1990. Early histogenesis of the teleostean retina: studies using a novel immunochemical marker, proliferating cell nuclear antigen (PCNA/cyclin). Dev. Brain Res. 55, 121-125.

Negishi, K., Stell, W.K., Teranishi, T., Karkhanis, A., Owusu-Yaw, V., Takasaki, Y., 1991a. Induction of proliferating cell nuclear antigen (PCNA)-immunoreactive cells in goldfish retina following intravitreal injection with 6hydroxydopamine. Cell. Mol. Neurobiol. 11, 639-659.

Negishi, K., Sugawara, K., Shinagawa, S., Teranishi, T., Kuo, C.H., Takasaki, Y., 1991b. Induction of immunoreactive proliferating cell nuclear antigen (PCNA) in goldfish retina following intravitreal injection with tunicamycin. Dev. Brain Res. $63,71-83$.

Negishi, K., Teranishi, T., Kato, S., 1982a. Neurotoxic destruction of dopaminergic cells in the carp retina revealed by a histoflourescence study. Acta Histochem. Cytochem. 15 (6), 768-778.

Negishi, K., Teranishi, T., Kato, S., 1982b. New dopaminerpic and indoleamineaccumulating cells in the growth zone of goldfish retinas after neurotoxic destruction. Science 216, 747-749.

Negishi, K., Teranishi, T., Kato, S., Nakamura, Y., 1987. Paradoxical induction of dopaminergic cells following intravitreal injection of high doses of 6hydroxydopamine in juvenile carp retina. Dev. Brain Res. 33, 67-79.

Nelson, B.R., Ueki, Y., Reardon, S., Karl, M.O., Georgi, S., Hartman, B.H., Lamba, D.A. Reh, T.A., 2011. Genome-wide analysis of Müller glial differentiation reveals a requirement for Notch signaling in postmitotic cells to maintain the glial fate. PLoS One 6, e22817.

Nelson, C.M., Ackerman, K.M., O’Hayer, P., Bailey, T.J., Gorsuch, R.A., Hyde, D.R., 2013. Tumor necrosis factor-alpha is produced by dying retinal neurons and is required for Müller glia proliferation during zebrafish retinal regeneration. J. Neurosci. 33, 6524-6539.

Nelson, C.M., Gorsuch, R.A., Bailey, T.J., Ackerman, K.M., Kassen, S.C., Hyde, D.R., 2012. Stat3 defines three populations of Müller glia and is required for initiating maximal Müller glia proliferation in the regenerating zebrafish retina. J. Comp. Neurol. 520, 4294-4311.

Nelson, S.M., Frey, R.A., Wardwell, S.L., Stenkamp, D.L., 2008. The developmental sequence of gene expression within the rod photoreceptor lineage in embryonic zebrafish. Dev. Dyn. 237, 2903-2917.

Niwa, H., Burdon, T., Chambers, I., Smith, A., 1998. Self-renewal of pluripotent embryonic stem cells is mediated via activation of STAT3. Genes Dev. 12, 20482060.

Noctor, S.C., Flint, A.C., Weissman, T.A., Dammerman, R.S., Kriegstein, A.R., 2001. Neurons derived from radial glial cells establish radial units in neocortex. Nature 409, 714-720.

Noctor, S.C. Flint, A.C., Weissman, T.A., Wong W.S, Clinton, B.K. Kriegstein, A.R. 2002. Dividing precursor cells of the embryonic cortical ventricular zone have morphological and molecular characteristics of radial glia. J. Neurosci. 22, $3161-3173$.

Norden, C., Young, S., Link, B.A., Harris, W.A., 2009. Actomyosin is the main driver of interkinetic nuclear migration in the retina. Cell 138, 1195-1208.

Ochocinska, M.J., Hitchcock, P.F., 2007. Dynamic expression of the basic helixloop-helix transcription factor neuroD in the rod and cone photoreceptor lineages in the retina of the embryonic and larval zebrafish. J. Comp. Neurol. $501,1-12$. 
Ochocinska, M.J., Hitchcock, P.F., 2009. NeuroD regulates proliferation of photoreceptor progenitors in the retina of the zebrafish. Mech. Dev. 126, 128-141.

Ohata, S., Aoki, R., Kinoshita, S., Yamaguchi, M., Tsuruoka-Kinoshita, S., Tanaka, H., Wada, H., Watabe, S., Tsuboi, T., Masai, I., Okamoto, H., 2011. Dual roles of notch in regulation of apically restricted mitosis and apicobasal polarity of neuroepithelial cells. Neuron 69, 215-230.

Okano, H., Temple, S., 2009. Cell types to order: temporal specification of CNS stem cells. Curr. Opin. Neurobiol. 19, 112-119.

Osakada, F., Ikeda, H., Mandai, M., Wataya, T., Watanabe, K., Yoshimura, N., Akaike, A., Sasai, Y., Takahashi, M., 2008. Toward the generation of rod and cone photoreceptors from mouse, monkey and human embryonic stem cells. Nat. Biotechnol. 26, 215-224.

Otteson, D., Cirenza, P., Hitchcock, P., 2002. Persistent neurogenesis in the teleost retina: evidence for regulation by the growth-hormone/insulin-like growth factor-I axis. Mech. Dev. 117, 137.

Otteson, D.C., D'Costa, A.R., Hitchcock, P.F. 2001. Putative stem cells and the lineage of rod photoreceptors in the mature retina of the goldfish. Dev. Biol. 232, 62-76.

Otteson, D.C., Hitchcock, P.F., 2003. Stem cells in the teleost retina: persistent neurogenesis and injury-induced regeneration. Vis. Res. 43, 927-936.

Perron, M., Harris, W.A., 2000. Retinal stem cells in vertebrates. Bioessays 22, 685688

Peterson, R.E., Fadool, J.M., McClintock, J., Linser, P.J., 2001. Müller cell differentiation in the zebrafish neural retina: evidence of distinct early and late stages in cell maturation. J. Comp. Neurol. 429, 530-540.

Picard, E., Ranchon-Cole, I., Jonet, L., Beaumont, C., Behar-Cohen, F., Courtois, Y., Jeanny, J.C., 2011. Light-induced retinal degeneration correlates with changes in iron metabolism gene expression, ferritin level, and aging. Investig. Ophthalmol. Vis. Sci. 52, 1261-1274.

Plath, K., Lowry, W.E., 2011. Progress in understanding reprogramming to the induced pluripotent state. Nat. Rev. Genet. 12, 253-265.

Politi, L., Rotstein, N., Carri, N., 2001. Effects of docosahexaenoic acid on retinal development: cellular and molecular aspects. Lipids 36, 927-935.

Pollak, J., Wilken, M.S., Ueki, Y., Cox, K.E., Sullivan, J.M., Taylor, R.J., Levine, E.M., Reh, T.A., 2013. ASCL1 reprograms mouse Müller glia into neurogenic retinal progenitors. Development 140, 2619-2631.

Powell, C., Elsaeidi, F., Goldman, D., 2012. Injury-dependent Müller glia and ganglion cell reprogramming during tissue regeneration requires Apobec2a and Apobec2b. J. Neurosci. 32, 1096-1109.

Powers, M.K., Bassi, C.J., Rone, L.A., Raymond, P.A., 1988. Visual detection by the rod system in goldfish of different sizes. Vis. Res. 28, 211-221.

Prada, F.A., Magalhaes, M.M., Coimbra, A., Genis-Galvez, J.M., 1989. Morphological differentiation of the Müller cell: golgi and electron microscopy study in the chick retina. J. Morphol. 201, 11-22.

Puro, D.G., 1995. Growth factors and Müller cells. Prog. Retin Eye Res. 15, 89-101.

Qin, Z., Barthel, L.K., Raymond, P.A., 2009. Genetic evidence for shared mechanisms of epimorphic regeneration in zebrafish. Proc. Natl. Acad. Sci. U. S. A. 106, 93109315.

Qin, Z., Kidd 3rd, A.R., Thomas, J.L., Poss, K.D., Hyde, D.R., Raymond, P.A., Thummel, R., 2011. FGF signaling regulates rod photoreceptor cell maintenance and regeneration in zebrafish. Exp. Eye Res. 93, 726-734.

Rakic, P., 1972. Mode of cell migration to the superficial layers of fetal monkey neocortex. J. Comp. Neurol. 145, 61-83.

Rakic, P., 2003. Elusive radial glial cells: historical and evolutionary perspective. Glia $43,19-32$.

Ramachandran, R., Fausett, B.V., Goldman, D., 2010a. Ascl1a regulates Müller glia dedifferentiation and retinal regeneration through a Lin-28-dependent, let-7 microRNA signalling pathway. Nat. Cell Biol. 12, 1101-1107.

Ramachandran, R., Reifler, A., Parent, J.M., Goldman, D., 2010b. Conditional gene expression and lineage tracing of tuba1a expressing cells during zebrafish development and retina regeneration. J. Comp. Neurol. 518, 4196-4212.

Ramachandran, R., Zhao, X.-F., Goldman, D., 2011. Ascl1a/Dkk/ $\beta$-catenin signaling pathway is necessary and glycogen synthase kinase- $3 \beta$ inhibition is sufficient for zebrafish retina regeneration. Proc. Natl. Acad. Sci. U. S. A. 108, 15858-15863.

Ramachandran, R., Zhao, X.F., Goldman, D., 2012. Insm1a-mediated gene repression is essential for the formation and differentiation of Müller glia-derived progenitors in the injured retina. Nat. Cell Biol. 14, 1013-1023.

Ramón y Cajal, S., 1960. Development of the Constituent Elements of the Retina, Studies on Vertebrate Neurogenesis. Charles C. Thomas, Springfield, IL, pp. 353-401.

Ramón y Cajal, S., 1972. The Structure of the Retina. Charles C. Thomas, Springfield, IL.

Rapaport, D.H., Rakic, P., LaVail, M.M., 1996. Spatiotemporal gradients of cell genesis in the primate retina. Perspect. Dev. Neurobiol. 3, 147-159.

Rapaport, D.H., Robinson, S.R., Stone, J., 1984. Cell movement and birth of the developing cat retina. In: Stone, J., Dreher, B., Rapaport, D.H. (Eds.), Development of Visual Pathways in Mammals. A. R. Liss, Syndey, Australia, pp. 23-38.

Rapaport, D.H., Wong, L.L., Wood, E.D., Yasumura, D., LaVail, M.M., 2004. Timing and topography of cell genesis in the rat retina. J. Comp. Neurol. 474, 304-324.

Raymond, P.A., 1985. Cytodifferentiation of photoreceptors in larval goldfish: delayed maturation of rods. J. Comp. Neurol. 236, 90-105.

Raymond, P.A., 1986. Movement of retinal terminals in goldfish optic tectum predicted by analysis of neuronal proliferation. J. Neurosci. 6, 2479-2488.

Raymond, P.A., 1991. Retinal regeneration in teleost fish. Ciba Found. Symp. 160, $171-186$.

Raymond, P.A., 2004. Restoration of vision. In: Lanza, R. Blau, H., Melton, D. Moore, M., Thomas, E.D., Verfaillie, C., Weissman, I., West, M. (Eds.), Handbook of Stem Cells. Elsevier Academic Press, Burlington, MA, pp. 703-710.
Raymond, P.A., Barthel, L.K., Bernardos, R.L., Perkowski, J.J., 2006. Molecular characterization of retinal stem cells and their niches in adult zebrafish. BMC Dev. Biol. 6, 36.

Raymond, P.A., Barthel, L.K., Curran, G.A., 1995. Developmental patterning of rod and cone photoreceptors in embryonic zebrafish. J. Comp. Neurol. 359, $537-$ 550.

Raymond, P.A., Barthel, L.K., Rounsifer, M.E., Sullivan, S.A., Knight, J.K., 1993. Expression of rod and cone visual pigments in goldfish and zebrafish: a rhodopsin-like gene is expressed in cones. Neuron 10, 1161-1174.

Raymond, P.A., Hitchcock, P.F., 1997. Retinal regeneration: common principles but a diversity of mechanisms. Adv. Neurol. 72, 171-184.

Raymond, P.A., Hitchcock, P.F., 2000. How the neural retina regenerates. In: Fini, M.E. (Ed.), Vertebrate Eye Development. Springer-Verlag, Berlin Heidelberg, pp. 197-218.

Raymond, P.A., Hitchcock, P.F., Palopoli, M.F., 1988a. Neuronal cell proliferation and ocular enlargement in Black Moor goldfish. J. Comp. Neurol. 276, 231-238.

Raymond, P.A., Reifler, M.J., Rivlin, P.K., 1988b. Regeneration of goldfish retina: rod precursors are a likely source of regenerated cells. J. Neurobiol. 19, 431-463.

Raymond, P.A., Rivlin, P.K., 1987. Germinal cells in the goldfish retina that produce rod photoreceptors. Dev. Biol. 122, 120-138.

Reh, T.A., Levine, E.M., 1998. Multipotential stem cells and progenitors in the vertebrate retina. J. Neurobiol. 36, 206-220.

Reichenbach, A., Bringmann, A., 2013. New functions of Müller cells. Glia 61, 651678.

Revishchin, A.V., Korochkin, L.I., Okhotin, V.E., Pavlova, G.V., 2008. Neural stem cells in the mammalian brain. Int. Rev. Cytol. 265, 55-109.

Rich, K.A., Figueroa, S.L., Zhan, Y., Blanks, J.C., 1995. Effects of Müller cell disruption on mouse photoreceptor cell development. Exp. Eye Res. 61, 235-248.

Robel, S., Berninger, B., Gotz, M., 2011. The stem cell potential of glia: lessons from reactive gliosis. Nat. Rev. Neurosci. 12, 88-104.

Roesch, K., Jadhav, A.P., Trimarchi, J.M., Stadler, M.B., Roska, B., Sun, B.B., Cepko, C.L. 2008. The transcriptome of retinal Müller glial cells. J. Comp. Neurol. 509, 225-238.

Rohrer, B., Stell, W.K., 1994. Basic fibroblast growth factor (bFGF) and transforming growth factor beta (TGF-Beta) act as stop and go signals to modulate postnatal ocular growth in the chick. Exp. Eye Res. 58, 553-562.

Salbreux, G., Barthel, L.K., Raymond, P.A., Lubensky, D.K., 2012. Coupling mechanical deformations and planar cell polarity to create regular patterns in the zebrafish retina. PLoS Comput. Biol. 8, e1002618.

Sandy, J.M., Blaxter, J.H.S., 1980. A study of retinal development in larvae herring and sole. J. Mar. Biol. Assoc. (U. K.) 60, 59-71.

Sarthy, P.V., 1985. Establishment of Müller cell cultures from adult rat retina. Brain Res. 337, 138-141.

Sarthy, V., 1991. Reactive gliosis in retinal degenerations. In: Anderson, R.E, Hollyfield, J.G., LaVail, M.M. (Eds.), Retinal Degenerations. CRC Press, Boca Raton, FL, pp. 109-116.

Sarthy, V.J., Ripps, H., 2001. The Retinal Müller Cell: Structure and Function. Kluwer Academic Publisher, Hingham, MA.

Sato, N., Sanjuan, I.M., Heke, M., Uchida, M., Naef, F., Brivanlou, A.H., 2003. Molec ular signature of human embryonic stem cells and its comparison with the mouse. Dev. Biol. 260, 404-413.

Sauer, F., 1935. Mitosis in the neural tube. J. Comp. Neurol. 62 (377), 405.

Scheer, N., Groth, A., Hans, S., Campos-Ortega, J.A., 2001. An instructive function for Notch in promoting gliogenesis in the zebrafish retina. Development 128 1099-1107.

Schmitt, E.A., Dowling, J.E., 1996. Comparison of topographical patterns of ganglion and photoreceptor cell differentiation in the retina of the zebrafish, Danio rerio. J. Comp. Neurol. 371, 222-234.

Schmitt, E.A., Dowling, J.E., 1999. Early retinal development in the zebrafish, Danio rerio: light and electron microscopic analyses. J. Comp. Neurol. 404, 515-536.

Senut, M.C., Gulati-Leekha, A., Goldman, D., 2004. An element in the alpha1-tubulin promoter is necessary for retinal expression during optic nerve regeneration but not after eye injury in the adult zebrafish. J. Neurosci. 24, 7663-7673.

Shaham, O., Menuchin, Y., Farhy, C., Ashery-Padan, R., 2012. Pax6: a multi-level regulator of ocular development. Prog. Retin Eye Res. 31, 351-376.

Shen, W., Fruttiger, M., Zhu, L., Chung, S.H., Barnett, N.L., Kirk, J.K., Lee, S. Coorey, N.J., Killingsworth, M., Sherman, L.S., Gillies, M.C., 2012. Conditiona Müller cell ablation causes independent neuronal and vascular pathologies in a novel transgenic model. J. Neurosci. 32, 15715-15727.

Sherpa, T., Fimbel, S.M., Mallory, D.E., Maaswinkel, H., Spritzer, S.D., Sand, J.A., Li, L. Hyde, D.R., Stenkamp, D.L., 2008. Ganglion cell regeneration following wholeretina destruction in zebrafish. Dev. Neurobiol. 68, 166-181.

Sherpa, T., Hunter, S.S., Frey, R.A., Robison, B.D., Stenkamp, D.L., 2011. Retinal proliferation response in the buphthalmic zebrafish, bugeye. Exp. Eye Res. 93, 424-436

Shimojo, H., Ohtsuka, T., Kageyama, R., 2008. Oscillations in notch signaling regulate maintenance of neural progenitors. Neuron 58, 52-64.

Sidman, R.L., 1960. Histogenesis of mouse retina studied with thymidine-H3. In: Smelser, G.K. (Ed.), The Structure of the Eye. Academic Press, New York, pp. 487-506.

Singh, A., Tsonis, P.A., 2010. Focus on molecules: six3-master or apprentice? Exp. Eye Res. 90, 535-536.

Sivak, J.M., Fini, M.E., 2002. MMPs in the eye: emerging roles for matrix metalloproteinases in ocular physiology. Prog. Retin Eye Res. 21, 1-14.

Stenkamp, D.L., 2007. Neurogenesis in the fish retina. Int. Rev. Cytol. 259, 173-224.

Stenkamp, D.L., 2011. The rod photoreceptor lineage of teleost fish. Prog. Retin Eye Res. 30, 395-404. 
Stenkamp, D.L., Barthel, L.K., Raymond, P.A., 1997. Spatiotemporal coordination of rod and cone photoreceptor differentiation in goldfish retina. J. Comp. Neurol. $382,272-284$

Stenkamp, D.L., Cameron, D.A., 2002. Cellular pattern formation in the retina: retinal regeneration as a model system. Mol. Vis. 8, 280-293.

Stenkamp, D.L., Hisatomi, O., Barthel, L.K., Tokunaga, F., Raymond, P.A., 1996. Temporal expression of rod and cone opsins in embryonic goldfish retina predicts the spatial organization of the cone mosaic. Investig. Ophthalmol. Vis. Sci. 37, $363-376$.

Stenkamp, D.L., Powers, M.K., Carney, L.H., Cameron, D.A., 2001. Evidence for two distinct mechanisms of neurogenesis and cellular pattern formation in regenerated goldfish retinas. J. Comp. Neurol. 431, 363-381.

Stephens, W.Z., Senecal, M., Nguyen, M., Piotrowski, T., 2010. Loss of adenomatous polyposis coli (apc) results in an expanded ciliary marginal zone in the zebrafish eye. Dev. Dyn. 239, 2066-2077.

Stevens, C.B., Cameron, D.A., Stenkamp, D.L., 2011. Plasticity of photoreceptorgenerating retinal progenitors revealed by prolonged retinoic acid exposure. BMC Dev. Biol. 11, 51.

Stiemke, M.M., Hollyfield, J.G., 1995. Cell birthdays in Xenopus laevis retina. Differentiation 58, 189-193.

Straznicky, K., Gaze, R.M., 1971. The growth of the retina in Xenopus laevis: an autoradiographic study. J. Embryol. Exp. Morphol. 26 (67), 79.

Sullivan, S.A., Barthel, L.K., Largent, B.L., Raymond, P.A., 1997. A goldfish Notch-3 homologue is expressed in neurogenic regions of embryonic, adult, and regenerating brain and retina. Dev. Genet. 20, 208-223.

Surzenko, N., Crowl, T., Bachleda, A., Langer, L., Pevny, L., 2013. SOX2 maintains the quiescent progenitor cell state of postnatal retinal Müller glia. Development 140, 1445-1456.

Suzuki, S.C., Bleckert, A., Williams, P.R., Takechi, M., Kawamura, S., Wong, R.O. 2013. Cone photoreceptor types in zebrafish are generated by symmetric terminal divisions of dedicated precursors. Proc. Natl. Acad. Sci. U. S. A. 110 15109-15114.

Suzumura, A., Sawada, M., Yamamoto, H., Marunouchi, T., 1993. Transforming growth factor-beta suppresses activation and proliferation of microglia in vitro. J. Immunol. 151, 2150-2158.

Swaroop, A., Kim, D., Forrest, D., 2010. Transcriptional regulation of photoreceptor development and homeostasis in the mammalian retina. Nat. Rev. Neurosci. 11, $563-576$.

Takahashi, K., Tanabe, K., Ohnuki, M., Narita, M., Ichisaka, T., Tomoda, K., Yamanaka, S., 2007. Induction of pluripotent stem cells from adult human fibroblasts by defined factors. Cell 131, 861-872.

Takahashi, K., Yamanaka, S., 2006. Induction of pluripotent stem cells from mouse embryonic and adult fibroblast cultures by defined factors. Cell 126 $663-676$.

Takeda, M., Takamiya, A., Jiao, J.W., Cho, K.S., Trevino, S.G., Matsuda, T., Chen, D.F., 2008. alpha-Aminoadipate induces progenitor cell properties of Müller glia in adult mice. Investig. Ophthalmol. Vis. Sci. 49, 1142-1150.

Tanaka, E.M., Reddien, P.W., 2011. The cellular basis for animal regeneration. Dev. Cell 21, 172-185.

Temple, S., 2001. The development of neural stem cells. Nature 414, 112-117.

Thomas, J.L., Nelson, C.M., Luo, X., Hyde, D.R., Thummel, R., 2012a. Characterization of multiple light damage paradigms reveals regional differences in photoreceptor loss. Exp. Eye Res. 97, 105-116.

Thomas, J.L., Ochocinska, M.J., Hitchcock, P.F., Thummel, R., 2012b. Using the Tg(nrd: egfp)/albino zebrafish line to characterize in vivo expression of neurod. PLoS One 7, e29128.

Thummel, R., Bailey, T.J., Hyde, D.R., 2011. In vivo electroporation of morpholinos into the adult zebrafish retina. J. Vis. Exp., e3603.

Thummel, R., Enright, J.M., Kassen, S.C., Montgomery, J.E., Bailey, T.J., Hyde, D.R. 2010. Pax6a and Pax6b are required at different points in neuronal progenitor cell proliferation during zebrafish photoreceptor regeneration. Exp. Eye Res. 90 $572-582$.

Thummel, R., Kassen, S.C., Enright, J.M., Nelson, C.M., Montgomery, J.E., Hyde, D.R., 2008a. Characterization of Müller glia and neuronal progenitors during adult zebrafish retinal regeneration. Exp. Eye Res. 87, 433-444.

Thummel, R., Kassen, S.C., Montgomery, J.E., Enright, J.M., Hyde, D.R., 2008b. Inhibition of Müller glial cell division blocks regeneration of the light-damaged zebrafish retina. Dev. Neurobiol. 68, 392-408.

Turner, D.L., Cepko, C.L., 1987. A common progenitor for neurons and glia persists in rat retina late in development. Nature 328, 131-136.

Turner, D.L., Snyder, E.Y., Cepko, C.L., 1990. Lineage-independent determination of cell type in the embryonic mouse retina. Neuron 4, 833-845.

Ueki, Y., Reh, T.A., 2013. EGF stimulates Müller glial proliferation via a BMPdependent mechanism. Glia 61, 778-789.

Uga, S., Smelser, G.K., 1973. Electron microscopic study of the development of retinal müllerian cells. Investig. Ophthalmol. Vis. Sci. 12, 295-367.

Vandooren, J., Van den Steen, P.E., Opdenakker, G., 2013. Biochemistry and molecular biology of gelatinase B or matrix metalloproteinase-9 (MMP-9): the next decade. Crit. Rev. Biochem Mol. Biol. 48, 222-272.

Varelas, X., Samavarchi-Tehrani, P., Narimatsu, M., Weiss, A., Cockburn, K, Larsen, B.G., Rossant, J., Wrana, J.L., 2010. The Crumbs complex couples cell density sensing to Hippo-dependent control of the TGF-beta-SMAD pathway. Dev. Cell 19, 831-844.
Vazquez-Chona, F.R., Swan, A., Ferrell, W.D., Jiang, L., Baehr, W., Chien, W.M., Fero, M., Marc, R.E., Levine, E.M., 2011. Proliferative reactive gliosis is compatible with glial metabolic support and neuronal function. BMC Neurosci. 12, 98.

Vihtelic, T.S., Hyde, D.R., 2000. Light-induced rod and cone cell death and regeneration in the adult albino zebrafish (Danio rerio) retina. J. Neurobiol. 44, 289307.

Vihtelic, T.S., Soverly, J.E., Kassen, S.C., Hyde, D.R., 2006. Retinal regional differences in photoreceptor cell death and regeneration in light-lesioned albino zebrafish. Exp. Eye Res. 82, 558-575.

Vilter, V., Lewin, L., 1954. Existence et répartition des mitoses dans la rétine d'un poisson abyssal, Bathylagus benedicti. C. R. Soc. Biol. (Paris) 148, 1771-1775.

Vitorino, M., Jusuf, P.R., Maurus, D., Kimura, Y., Higashijima, S., Harris, W.A., 2009. Vsx2 in the zebrafish retina: restricted lineages through derepression. Neural Dev. 4,14

Wachs, F.P., Winner, B., Couillard-Despres, S., Schiller, T., Aigner, R., Winkler, J., Bogdahn, U., Aigner, L., 2006. Transforming growth factor-beta1 is a negative modulator of adult neurogenesis. J. Neuropathol. Exp. Neurol. 65, 358-370.

Wagers, A.J., 2012. The stem cell niche in regenerative medicine. Cell Stem Cell 10, $362-369$.

Wan, J., Ramachandran, R., Goldman, D., 2012. HB-EGF is necessary and sufficient for Müller glia dedifferentiation and retina regeneration. Dev. Cell 22, 334-347.

Wang, S.W., Mu, X., Bowers, W.J., Klein, W.H., 2002a. Retinal ganglion cell differentiation in cultured mouse retinal explants. Methods 28, 448-456.

Wang, Y., Smith, S.B., Ogilvie, J.M., McCool, D.J., Sarthy, V., 2002b. Ciliary neurotrophic factor induces glial fibrillary acidic protein in retinal Müller cells through the JAK/STAT signal transduction pathway. Curr. Eye Res. 24, 305312.

Wargelius, A., Seo, H.C., Austbo, L., Fjose, A., 2003. Retinal expression of zebrafish six3.1 and its regulation by Pax6. Biochem. Biophys. Res. Commun. 309, 475481.

Weber, A., Hochmann, S., Cimalla, P., Gartner, M., Kuscha, V., Hans, S., Geffarth, M., Kaslin, J., Koch, E., Brand, M., 2013. Characterization of light lesion paradigms and optical coherence tomography as tools to study adult retina regeneration in zebrafish. PLoS One 8, e80483.

Wehman, A.M., Staub, W., Meyers, J.R., Raymond, P.A., Baier, H., 2005. Genetic dissection of the zebrafish retinal stem-cell compartment. Dev. Biol. 281, 5365.

Wetts, R., Fraser, S.E., 1988. Multipotent precursors can give rise to all major cell types of the frog retina. Science 239, 1142-1145.

White, D.T., Mumm, J.S., 2013. The nitroreductase system of inducible targeted ablation facilitates cell-specific regenerative studies in zebrafish. Methods 62, 232-240.

Willbold, E., Reinicke, M., Lance-Jones, C., Lagenaur, C., Lemmon, V., Layer, P.G., 1995. Müller glia stabilizes cell columns during retinal development: lateral cell migration but not neuropil growth is inhibited in mixed chick-quail retinospheroids. Eur. J. Neurosci. 7, 2277-2284.

Williams, P.R., Morgan, J.L., Kerschensteiner, D., Wong, R.O., 2013. In vivo imaging of zebrafish retina. Cold Spring Harb. Protoc. 2013.

Wohl, S.G., Schmeer, C.W., Isenmann, S., 2012. Neurogenic potential of stem/ progenitor-like cells in the adult mammalian eye. Prog. Retin Eye Res. 31, $213-242$.

Wu, D.M., Schneiderman, T., Burgett, J., Gokhale, P., Barthel, L., Raymond, P.A., 2001. Cones regenerate from retinal stem cells sequestered in the inner nuclear layer of adult goldfish retina. Investig. Ophthalmol. Vis. Sci. 42, 2115-2124.

Xu, L.Z., Sanchez, R., Sali, A., Heintz, N., 1996. Ligand specificity of brain lipidbinding protein. J. Biol. Chem. 271, 24711-24719.

Xue, W., Cojocaru, R.I., Dudley, V.J., Brooks, M., Swaroop, A., Sarthy, V.P., 2011. Ciliary neurotrophic factor induces genes associated with inflammation and gliosis in the retina: a gene profiling study of flow-sorted, Müller cells. PLoS One 6 , e20326.

Xue, X.Y., Harris, W.A., 2012. Using myc genes to search for stem cells in the ciliary margin of the Xenopus retina. Dev. Neurobiol. 72, 475-490.

Young, R.W., 1985a. Cell differentiation in the retina of the mouse. Anat. Rec. 212, 199-205.

Young, R.W., 1985b. Cell proliferation during postnatal development of the retina in the mouse. Dev. Brain Res. 21, 229-239.

Yu, F.X., Guan, K.L., 2013. The Hippo pathway: regulators and regulations. Genes. Dev. 27, 355-371.

Yurco, P., Cameron, D.A., 2005. Responses of Müller glia to retinal injury in adult zebrafish. Vis. Res. 45, 991-1002.

Zhang, C., Li, H., Liu, M.G., Kawasaki, A., Fu, X.Y., Barnstable, C.J., Shao-Min Zhang, S., 2008. STAT3 activation protects retinal ganglion cell layer neurons in response to stress. Exp. Eye Res. 86, 991-997.

Zhang, S.S., Liu, M.G., Kano, A., Zhang, C., Fu, X.Y., Barnstable, C.J., 2005. STAT3 activation in response to growth factors or cytokines participates in retina precursor proliferation. Exp. Eye Res. 81, 103-115.

Zhao, B., Li, L., Lei, Q., Guan, K.L., 2010a. The Hippo-YAP pathway in organ size control and tumorigenesis: an updated version. Genes. Dev. 24, 862-874.

Zhao, C., Sun, G., Li, S., Lang, M.F., Yang, S., Li, W., Shi, Y., 2010b. MicroRNA let-7b regulates neural stem cell proliferation and differentiation by targeting nuclear receptor TLX signaling. Proc. Natl. Acad. Sci. U. S. A. 107, 1876-1881.

Zhong, W., Chia, W., 2008. Neurogenesis and asymmetric cell division. Curr. Opin. Neurobiol. 18, 4-11. 
Zuber, M.E., Gestri, G., Viczian, A.S., Barsacchi, G., Harris, W.A., 2003. Specification of the vertebrate eye by a network of eye field transcription factors. Development $130,5155-5167$.

Zupanc, G.K., 2001. Adult neurogenesis and neuronal regeneration in the central nervous system of teleost fish. Brain Behav. Evol. 58, 250-275.

Zupanc, G.K., 2006. Neurogenesis and neuronal regeneration in the adult fish brain. J. Comp. Physiol. Neuroethol. Sens. Neural Behav. Physiol. 192, 649-670.
Zupanc, G.K., Hinsch, K., Gage, F.H., 2005. Proliferation, migration, neuronal differentiation, and long-term survival of new cells in the adult zebrafish brain. J. Comp. Neurol. 488, 290-319.

Zupanc, G.K., Sirbulescu, R.F., 2011. Adult neurogenesis and neuronal regeneration in the central nervous system of teleost fish. Eur. J. Neurosci. 34, 917-929. 